\title{
DEVELOPMENT OF DYNAMIC LIGHT SCATTERING OPTICAL COHERENCE TOMOGRAPHY METHODS FOR DETECTING CELL DEATH
}

\author{
By \\ Nico Joseph John Arezza \\ B.Eng, Ryerson University, 2016 \\ Toronto, Ontario, Canada \\ A thesis \\ presented to Ryerson University \\ in partial fulfillment of the \\ requirements for the degree of \\ Master of Science \\ in the program of \\ Biomedical Physics
}

Toronto, Ontario, Canada, 2018

(C) Nico Joseph John Arezza, 2018 


\section{Author's Declaration}

I hereby declare that I am the sole author of this thesis. This is a true copy of the thesis, including any required final revisions, as accepted by my examiners.

I authorize Ryerson University to lend this thesis to other institutions or individuals for the purpose of scholarly research.

I further authorize Ryerson University to reproduce this thesis by photocopying or by other means, in total or in part, at the request of other institutions or individuals for the purpose of scholarly research.

I understand that my thesis may be made electronically available to the public.

Nico Joseph John Arezza 


\title{
Development of Dynamic Light Scattering Optical Coherence Tomography Methods for Detecting Cell Death
}

\author{
Master of Science \\ Nico Joseph John Arezza \\ Biomedical Physics, Ryerson University, 2018
}

\section{Abstract}

Dynamic light scattering (DLS) techniques can provide information about the quantity, size, and motion of light scatterers within a volume based on temporal fluctuations in its light scattering profile. In DLS, autocorrelation functions ( $\mathrm{ACFs}$ ) are computed from light intensity vs time signals acquired from optical imaging setups. A parameter known as the decorrelation time is computed from each ACF and is inversely related to the average motion speed of scatterers within the imaging volume. Optical coherence tomography is an imaging modality that generates $2 \mathrm{D}$ cross-sectional images based on light backscattered from a sample, and the combination of DLS with OCT is known as dynamic light scattering optical coherence tomography (DLS-OCT).

Previously, DLS-OCT has been used to detect apoptosis, a form of programmed cell death, in non-adherent leukemia cells. Cells undergoing apoptosis experience predictable morphological changes that results in an increase in intracellular motion, and therefore a decrease in decorrelation time. We applied DLS-OCT methods to quantify the decorrelation 
times in adherent breast cancer cell pellets that were either untreated, treated with $20 \mathrm{ng} / \mathrm{mL}$ paclitaxel for 24 or 48 hours, or deprived of media for 24 or 48 hours. The mean decorrelation times in the paclitaxel-treated and nutrient deprived groups were significantly lower than in the untreated cells $(\mathrm{p}<0.05)$, suggestive of increased intracellular motion due to morphological cellular changes associated with cell death.

We also investigated a new model to fit to ACFs generated by DLS-OCT of cell pellets. Typically, ACFs are fit to single exponential decay curves. We developed a model that expresses the ACFs from in vitro experiments as a sum of multiple exponential decay curves using an algorithm known as CONTIN. The curves produced by CONTIN fitted the experimental data much better than the single exponential decay fits. We speculate that the CONTIN fits, each of which resembled a superposition of three exponential decay functions, may result from light scattered from three different types of scatterers within cells, such as lysosomes, mitochondria, and nuclei. 


\section{Acknowledgements}

The work presented herein would not have been possible without the assistance of several people.

I would first like to thank my supervisor, Dr. Michael C. Kolios, for his expertise and knowledge throughout the course of this research, and for the guidance and supervision that helped me develop into a more well-rounded and independent scientist. I have grown tremendously throughout my time as a M.Sc student, and much of this can be attributed to Dr. Kolios' mentorship, as well as the overall atmosphere of the Kolios lab. I would also like to thank my supervisory committee, Dr. Yuan Xu and Dr. Carl Kumaradas, for their insightful guidance and thought-provoking questions.

I would like to thank Dr. Marjan Razani, whose technical expertise ensured that experiments involving the OCT device always went smoothly. Her advice and wisdom, drawn from her own experiences, have also been extremely valuable.

I would like to thank Elizabeth Berndl, for her assistance with cell culturing and for training me to use the wet lab facilities.

I'd also like to thank Dr. Golnaz Farhat, Kirsten Cardinell, Dr. Jesse Tanguay, and the Kolios lab members.

Finally, I'd like to thank my parents for encouraging me to pursue my desires. Their sacrifices and support have made all of this possible. 


\section{Table of Contents}

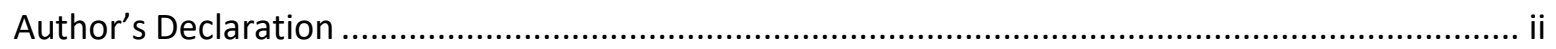

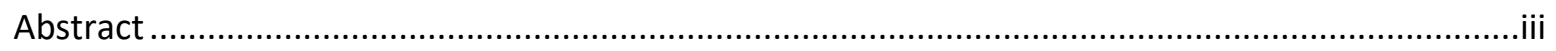

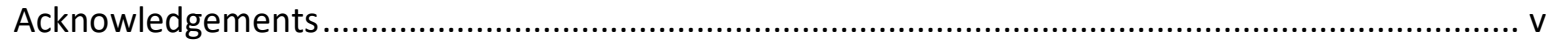

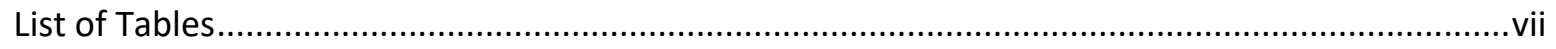

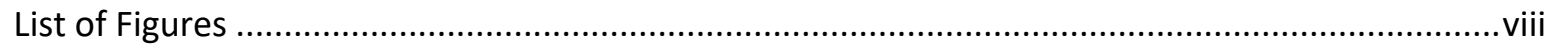

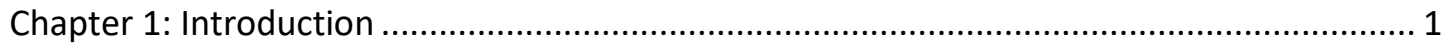

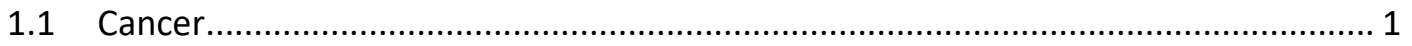

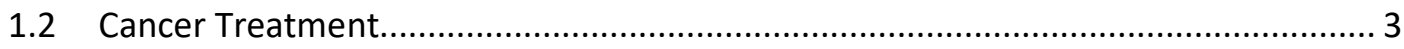

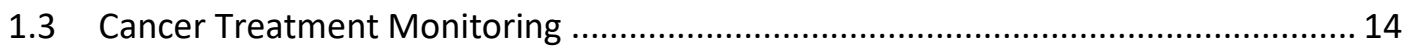

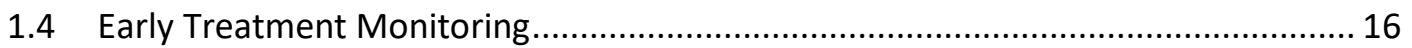

1.5 Detecting Cell Death with Optical Coherence Tomography .................................... 21

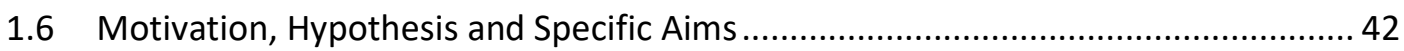

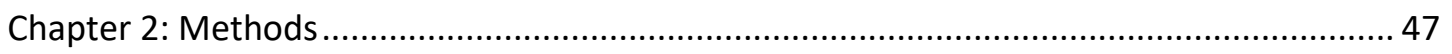

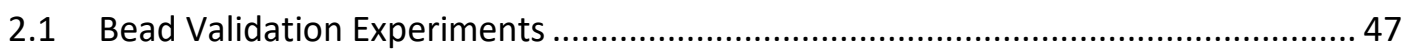

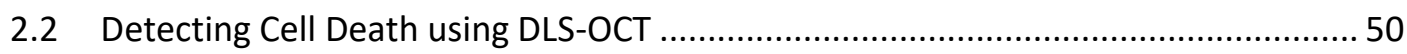

2.3 Using CONTIN to Determine Decorrelation Time Distributions.................................56

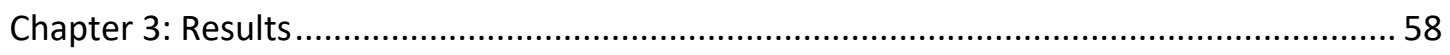

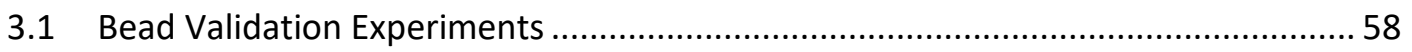

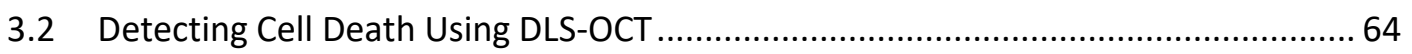

3.3 Using CONTIN to Determine Decorrelation Time Distributions ................................ 73

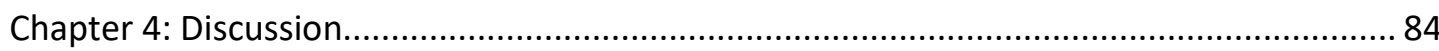

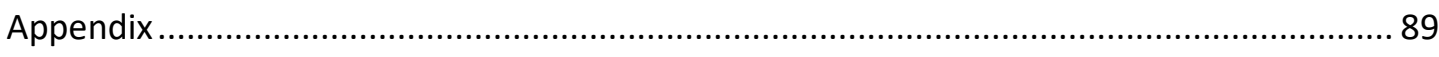

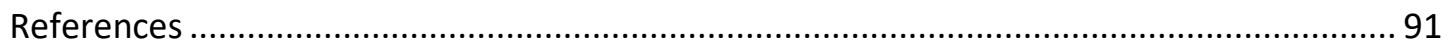




\section{List of Tables}

Table 1-1. Typical refractive indices for various organelles in a cell. (Adapted from [68])..... 44

Table 3-1. Average decorrelation times of paclitaxel-treated cells. Each trial took place on a different week and used cells from a different passage number. In brackets are the percent changes in decorrelation time from 0 hours to either 24 or 48 hours.

Table 3-2. Average decorrelation times of nutrient-deprived cells. Each trial took place on a different week and used cells from a different passage number. In brackets are the percent changes in decorrelation time from 0 hours to either 24 or 48 hours.

Table 3-3. Average AUCs the first peak of paclitaxel-treated cells. Each trial took place on a different week and used cells from a different passage number. In brackets are the percent changes in peak 1 AUC from 0 hours to either 24 or 48 hours. .76

Table 3-4. Average AUCs the third peak of paclitaxel-treated cells. Each trial took place on a different week and used cells from a different passage number. In brackets are the percent changes in peak 3 AUC from 0 hours to either 24 or 48 hours. .76

Table 3-5. Average AUCs the first peak of nutrient-deprived cells. Each trial took place on a different week and used cells from a different passage number. In brackets are the percent changes in peak 1 AUC from 0 hours to either 24 or 48 hours. .79

Table 3-6. Average AUCs the third peak of nutrient-deprived cells. Each trial took place on a different week and used cells from a different passage number. In brackets are the percent changes in peak 3 AUC from 0 hours to either 24 or 48 hours. .79

Table 3-7. Mean sum-of-squared-error of the exponential fitted curves and the CONTIN fitted curves. SSEs were calculated from ACFs containing 250 data points from time lags between $0 \mathrm{~ms}$ to $747 \mathrm{~ms}$. In brackets are the standard deviations across 30 measurements. 


\section{List of Figures}

Figure 1-1. Morphological changes associated with various methods of cell death. Apoptosis is characterized by fragmentation of the cell into membrane-bound apoptotic bodies, minimizing the release of inflammatory cellular contents. Oncosis is characterized by swelling of the cell and degradation of the plasma membrane, allowing the cell's contents to spill out into the extracellular space. In pyroptosis, the plasma membrane breaks down and cellular material spills out of the cell, accompanied by cytokines that trigger an immune response. Also shown in the image is autophagy, where parts of a cell are bound within vesicles and phagocytized. (Adapted from Fink et al $[11])$ .5

Figure 1-2. Schematic of the structural changes associated with the six stages of mitosis. First, the chromosomes are duplicated to form two identical copies. Next, the nuclear envelope degrades, and spindle fibers attach to the chromosomes and align them in the middle of the cell. The chromosomes are then separated into two identical groups and pulled to opposite sides of the cell. Finally, new nuclear envelopes form around the two chromosome clusters. After mitosis, the cell undergoes cytokinesis. (Adapted from the National Institute of General Medical Sciences [15]).......................8

Figure 1-3. Direct and indirect methods of radiation damage. Indirect action accounts for approximately $70 \%$ of DNA damage in radiation therapy. (Adapted from Nias [18]).......................10

Figure 1-4. The cell cycle. Though most healthy human cells are not dividing and exist in "out of cycle" states (G0 phase), some cell types such as bone marrow, skin, and cancer, are always proliferating. During the G1 phase, cells grow. The S phase is when DNA is replicated so that all chromosomes are doubled to form two pairs of sister chromatids. The cell continues to grow during the G2 phase, and mitotic spindles start to form. The M phase is classified by mitosis and cytokinesis. There are a number of biomarkers that can indicate which phase a cell is currently in, including Mcm2, Plk 1, Aurora A, geminin, and H3p. (Adapted from Williams and Stoeber [23]). .13

Figure 1-5. X-ray computed tomography (CT) image of a person's thoracic cavity in the transverse plane. Two cancerous lesions have been identified and measured with calipers. A large lesion is in 
the left lung and has a largest diameter shown by the A-B line, while a smaller lesion is located near the heart and has a largest diameter shown by the F-G line. RECIST only requires the largest diameter of each lesion, so the C-D line is not included in SAD calculations. (Adapted from Therasse et al [24]) 15

Figure 1-6. Resolution and penetration of various imaging modalities. Confocal microscopy and multiphoton microscopy (MPM) have very high spatial resolution but low penetration depth compared to OCT. Photoacoustic tomography (PAT), US, and MRI have better penetration than OCT but lower spatial resolution. (Adapted from UBC Biomedical Engineering [41])

Figure 1-7. Simplified schematic of an OCT instrument based on the Michelson interferometer. Light from the source is split into two paths: one to a reference mirror and the other to the sample. Then, the light reflected by the mirror recombines with the light backscattered by the sample, creating an interference signal. (Adapted from Brezinski [44]).

Figure 1-8. Schematic representation of a typical DLS setup. The source (L) illuminates the sample (S) with light, which is scattered. Some of the scattered light is captured by a photodetector (PD) which then inputs the signal to a correlator (COR). (Adapted from Goldburg [46])......

Figure 1-9. Size distributions of a latex solution generated using CONTIN. The actual solution contained $60 \mathrm{~nm}$ and $220 \mathrm{~nm}$ spheres. When a lower regularizer was used (green), CONTIN produced two distinct peaks for the $60 \mathrm{~nm}$ and $220 \mathrm{~nm}$ spheres. When higher regularizers were used (blue and red), the two populations were blended into a single peak. (Adapted from Malvern Panalytical [54])

Figure 1-10. Conceptual illustration of OCT resolution volume and light scattering contributions to DLS-OCT theory. Particles within an OCT resolution volume can be categorized into three groups at any point in time: static, moving, or entering and exiting an imaging voxel. (Adapted from Lee et al [56]) .33

Figure 1-11. The different light scattering regimes as a function of particle size and incident light wavelength. The ratio $x$ is equal to $2 \pi r / \lambda$ where $r$ is the radius of the particle and $\lambda$ is the incident light wavelength. (Adapted from Petty [61]). 
Figure 1-12. Scattering profiles of Rayleigh and Mie scattering regimes. Rayleigh scattering results in approximately the same intensity of light scattered in the forward direction and backward direction. In Mie scattering, however, significantly more light is scattered forward. (Adapted from [62]).........38

Figure 1-13. Results of DLS-OCT analysis of AML cells treated with cisplatin. (a): The intermediate scattering functions, which are the processed ACFs, from a control sample and three treated samples at different time points. (b): Decorrelation time as a function of treatment time. Error bars represent the standard deviation of 10 separate measurements from each sample. (Adapted from [43])...........40

Figure 2-1. High speed MEMS-VCSEL Swept Source OCT system from Thorlabs, Inc. (Adapted from Zam et al [74]).

Figure 2-2. The effects of various concentrations of paclitaxel on MCF-7 breast cancer cells. At concentrations higher than $20 \mathrm{ng} / \mathrm{mL}$, there is no significant increase in the percent of apoptotic cells. (Adapted from [77]).

Figure 2-3. (a): OCT B-mode image of an untreated MCF-7 cancer cell pellet. The image depicts a cross-sectional view of the cell pellet. The ROI is outlined by the red rectangle. (b): Signal intensity as a function of time for a single pixel in the ROI from (a).

Figure 2-4. CONTIN distributions of an ACF from untreated breast cancer cells. When the regularizer was set to 0 (left), there were overlapping peaks, but when the regularizer was set to 0.1 (right), all peaks were clearly defined and separated.

Figure 3-1. OCT cross-sectional images of monodisperse bead solutions. From left to right: $0.202 \mu \mathrm{m}$ diameter beads, $0.465 \mu \mathrm{m}$ diameter beads, and $0.989 \mu \mathrm{m}$ diameter beads. Image intensity is normalized on a scale from 0 (black) to 255 (white). .58

Figure 3-2. a): Average ACFs obtained from monodisperse microsphere solutions. b): Decorrelation time vs. particle diameter for DLS-OCT analysis of monodisperse microsphere solutions. Error bars represent the standard deviation from measurements at three spatial locations. The dotted line depicts the predicted decorrelation times as per the Einstein-Stokes equation. .59 
Figure 3-3. OCT cross-sectional image of the polydisperse microsphere solution containing $0.51 \mu \mathrm{m}$ and $1.93 \mu \mathrm{m}$ diameter beads mixed at a 1:1 volume ratio

Figure 3-4. Average ACFs obtained from monodisperse solutions of $0.53 \mu \mathrm{m}$ and $1.93 \mu \mathrm{m}$ diameter beads, and polydisperse solution containing both bead sizes. .60

Figure 3-5. Decorrelation time distribution functions for polystyrene bead solutions. The distributions were computed with CONTIN, using a regularizer of 0.

Figure 3-6. Decorrelation time distribution functions for polystyrene bead solutions. The distributions were computed with CONTIN, using a regularizer of 0.1

Figure 3-7. Normalized mean ACFs from cells that have been treated with paclitaxel. Each curve represents the average of $10 \mathrm{ACFs}$ from different points in a sample, which in turn represents the average ACF from 300 pixels within a ROI.

Figure 3-8. Decorrelation time as a function of paclitaxel treatment time. Each line (green, black, and magenta) represents a different trial of the same repeated experiment, all performed on separate weeks and with different cell samples. The error bars represent the standard deviation of 10 measurements.66

Figure 3-9. Decorrelation time frequency histograms for cells treated with paclitaxel. For each treatment duration, 30 decorrelation times were acquired, with 10 from each experimental trial. Bins are $5 \mathrm{~ms}$ wide .68

Figure 3-10. Normalized mean ACFs from cells that have been deprived of nutrients. Each curve represents the average of 10 ACFs from different points in a sample, which in turn represents the average ACF from 300 pixels within a ROI.

Figure 3-11. Decorrelation time as a function of nutrient deprivation time. Each line (green, black, and magenta) represents a different trial of the same repeated experiment, all performed on separate weeks and with different cell samples. The error bars represent the standard deviation of 10 measurements .70 
Figure 3-12. Decorrelation time frequency histograms for cells deprived of nutrients. For each treatment duration, 30 decorrelation times were acquired, with 10 from each experimental trial. Bins are $5 \mathrm{~ms}$ wide

Figure 3-13. Sample decorrelation time distribution functions for cell pellets made from cells treated with $20 \mathrm{ng} / \mathrm{mL}$ paclitaxel for various durations. Top: untreated cells, Centre: cells treated for 24 hours, Bottom: cells treated for 48 hours. Each of the above distributions came from one of the $30 \mathrm{ACFs}$ acquired for each treatment duration. .75

Figure 3-14. Sample decorrelation time distribution functions for cell pellets deprived of nutrients for various durations. Top: cells not deprived of nutrients, Centre: cells deprived of nutrients for 24 hours, Bottom: cells deprived of nutrients for 48 hours. Each of the above distributions came from one of the $30 \mathrm{ACF}$ acquired for each treatment duration .78

Figure 3-15. Exponential fit of a DLS-OCT ACF acquired from a pellet of untreated breast cancer cells. The experimental ACF is solid and the fitted curve is dashed. .82

Figure 3-16. CONTIN fit of a DLS-OCT ACF acquired from untreated breast cancer cells. The experimental ACF is solid and the fitted curve is dashed .83 


\section{Chapter 1: Introduction}

\subsection{Cancer}

Cancer is a group of diseases characterized by the uncontrolled growth of abnormal cells that typically form localized masses called tumors and can spread to distant parts of the body. In healthy tissues, cell division is a highly regulated process that works to maintain homeostasis through wound repair, cellular quality control, and growth; but in cancer cells, this process is unregulated [1]. Cancer can originate from many different tissues and there are physiological variations between different types of cancer. However, there are some traits shared by most cancers, six of which are described in The Hallmarks of Cancer [2] by D. Hanahan and R. Weinberg: cancer cells can (1) stimulate their own growth, (2) resist growthinhibition signals, (3) resist programmed cell death, (4) multiply indefinitely, (5) stimulate the growth of blood vessels to provide themselves with nutrients, and (6) invade local tissues and spread to distant sites, a process known as metastasis.

The symptoms experience by a cancer patient depend on the type of cancer they have and where there are tumors located in their body. As cancerous tumors grow, they cause local and systemic symptoms by applying physical pressure to their surroundings, creating an acidic extracellular microenvironment [3], and disrupting normal tissue function with hormones and intercellular signaling. Left untreated, cancer typically results in death.

It is estimated that approximately 1 in 2 Canadians will develop cancer in their lifetime and approximately 1 in 4 will die of the disease [4]. The risk of developing cancer increases with age [5]. Since the Canadian population is ageing due to an increasing average 
life expectancy and a sub-replacement fertility rate [6], the number of new incidences of cancer is expected to increase each year. 


\subsection{Cancer Treatment}

The purpose of cancer treatment is to stop the propagation of cancer cells, which can be achieved by surgically removing the cells from the body or by killing them. Surgery to remove tumors is one of the major pillars of cancer care, and it is estimated that up to $80 \%$ of all cancer cases worldwide require surgery to achieve the best patient outcome [7]. However, surgery cannot be performed on tumors that are inoperable, which means they are either too large to be removed, too complex, or embedded in sensitive organs. Furthermore, surgery does not remove undetected cancer cells that have metastasized away from a detected tumor. Treatments that induce cancer cell death attempt to overcome these limitations and a person diagnosed with cancer will typically undergo a combination of treatments that work together synergistically.

\subsubsection{Cell Death}

Cell death is the event in which a cell permanently ceases to carry out its functions. There are many ways to induce cell death, so distinct modes of cell death are categorized morphologically rather than by their method of induction. The major modes of cell death in eukaryotic cells are apoptosis, oncosis, and pyroptosis, summarized below and depicted in Figure 1-1.

Apoptosis is a term that was coined by Kerr et al [8] in 1972 and describes a form of programmed cell death that only occurs in multi-cellular organisms. Apoptosis is genetically encoded and can be initiated by a cell when it has become sufficiently damaged such that its ability to safely perform its functions have been compromised. A cell undergoing apoptosis 
experiences predictable morphological changes, and these include cellular and nuclear condensation, compression of chromatin, and fragmentation of the nucleus, mitochondria, and other organelles. The cell membrane experiences budding and eventually fragments into small, tightly-packed vesicles known as apoptotic bodies which contain cytoplasm, organelles, and nuclear fragments. Apoptotic bodies are phagocytized by macrophages or other cells. Since apoptotic cells release their contents into plasma membrane-bound bodies rather than spilling their contents out of the cell, apoptosis usually proceeds without eliciting an inflammatory response [9].

Apoptosis can be divided into three distinct steps: the initiation phase, the commitment phase, and the execution phase. The initiation phase begins when a cell responds to an internal or external signal that tells it to initiate apoptosis, typically after it has either sustained considerable damage or has accumulated substantial genetic defects. The commitment phase is when apoptosis becomes irreversible, and is highly regulated by mitochondria, which release inter-membrane space proteins that bind to inhibitors-ofapoptosis to relieve their inhibitory effects [10]. In the execution phase, the cell undergoes all the morphological changes characteristic of apoptosis from cell condensation to the formation of apoptotic bodies. 


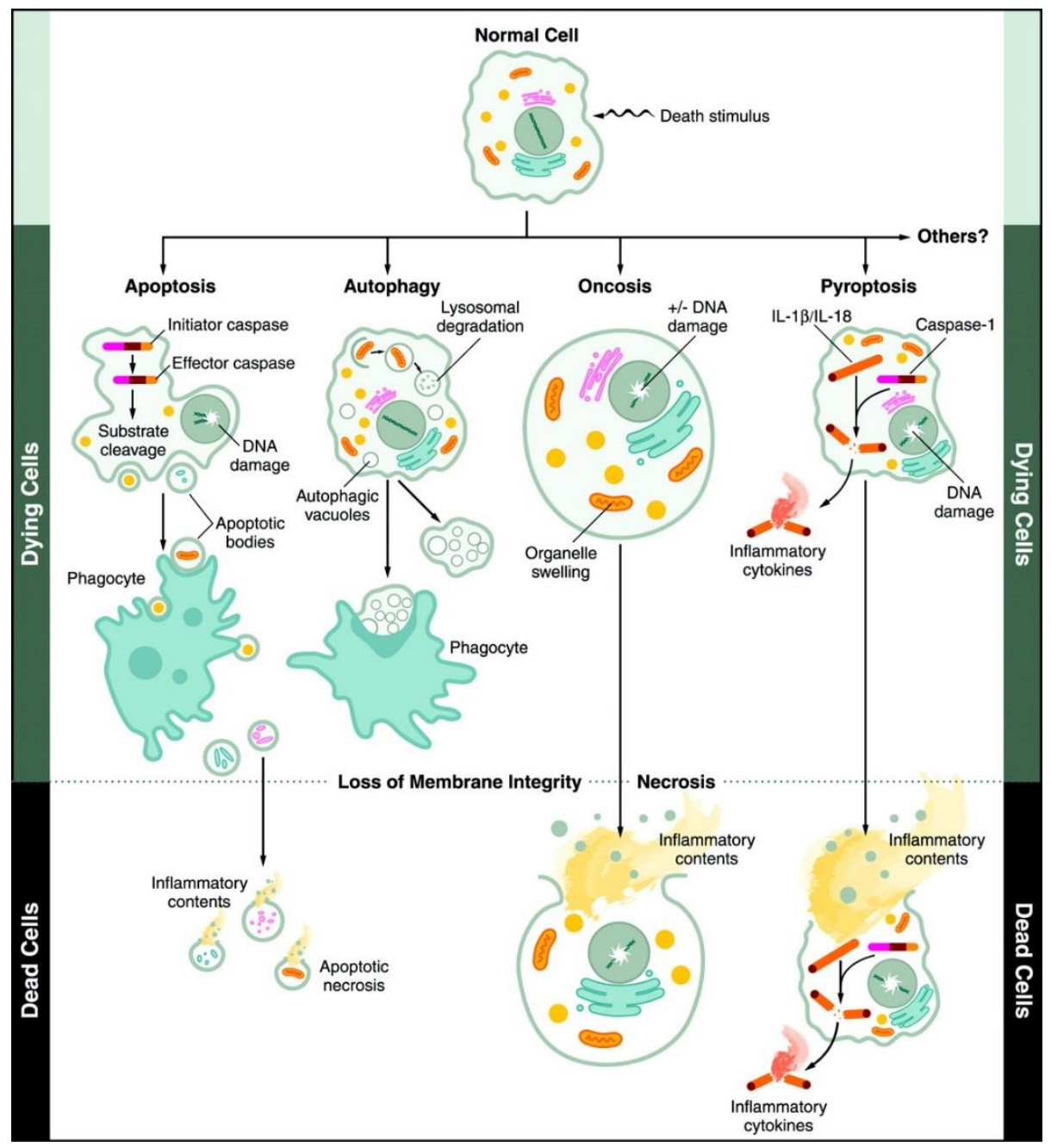

Figure 1-1. Morphological changes associated with various methods of cell death. Apoptosis is characterized by fragmentation of the cell into membrane-bound apoptotic bodies, minimizing the release of inflammatory cellular contents. Oncosis is characterized by swelling of the cell and degradation of the plasma membrane, allowing the cell's contents to spill out into the extracellular space. In pyroptosis, the plasma membrane breaks down and cellular material spills out of the cell, accompanied by cytokines that trigger an immune response. Also shown in the image is autophagy, where parts of a cell are bound within vesicles and phagocytized. (Adapted from Fink et al [11]).

Oncosis, also known as ischemic cell death, is a form of cell death largely caused by increased permeability of the plasma membrane, either due to mechanical trauma to the cell 
or energy depletion [12]. In viable cells, ion transporters use adenosine triphosphate (ATP) as an energy source to drive ions across the plasma membrane against an electro-chemical gradient. When a cell runs out of ATP, these pumps fail, which leads to increased membrane permeability. Ions can freely travel through the compromised plasma membrane and osmosis causes the cell and its organelles to swell. Eventually the cell experiences membrane blebbing and ruptures, and its contents are released into the extracellular space. Unlike apoptosis, oncosis elicits an inflammatory response.

Pyroptosis is an additional form of programmed cell death uniquely dependent on caspase-1, a protein-cleaving enzyme that initiates pyroptosis but is not involved in apoptosis or oncosis [11]. Pyroptosis can exhibit many of the same morphological features as oncosis, including cell swelling and membrane pore formation, but also results in the release of inflammatory cytokines that signal macrophages and other immune cells [11]. Pyroptosis is an important cell death mode in cells that have been infected by microbes or viruses, as the released cytokines attract immune cells to the infected area.

Mitotic arrest and mitotic catastrophe used to be classified as additional modes of cell death, but are now viewed as processes that lead to cell death by the more conventional methods of apoptosis and oncosis [13]. Both processes are related to mitosis, which is the stage in the cell cycle in which a cell's nucleus, the organelle containing a cell's deoxyribonucleic acid (DNA), is split into two separate nuclei.

During mitosis, the membrane surrounding a cell's nucleus, called the nuclear envelope, is degraded. The cell's chromosomes, which are tightly coiled DNA strands, are duplicated into sister chromatids. Structures known as mitotic spindles are formed from protein filaments and attach to the chromatids, eventually separating them into two separate 
but identical chromosome bundles. Finally, new nuclear envelopes form around the two chromosome bundles. After mitosis, the cell undergoes cytokinesis, where it divides into two daughter cells. The stages of mitosis are shown in detail in Figure 1-2.

Mitotic arrest occurs when the formation of spindle fibers in a mitotic cell is disrupted, rendering the cell unable to complete its division and leaving it in an arrested state. Cells cannot remain in mitotic arrest indefinitely and will eventually undergo apoptosis [13].

Mitotic catastrophe is an aberrant form of mitosis that typically results in the formation of nuclear envelopes around missegregated chromosomes in a cell [13]. It is associated with premature chromosome condensation and incomplete DNA synthesis during mitosis, both of which can result from radiation damage [14]. Cells that have undergone mitotic catastrophe have had their functions compromised and eventually undergo apoptosis or oncosis. 


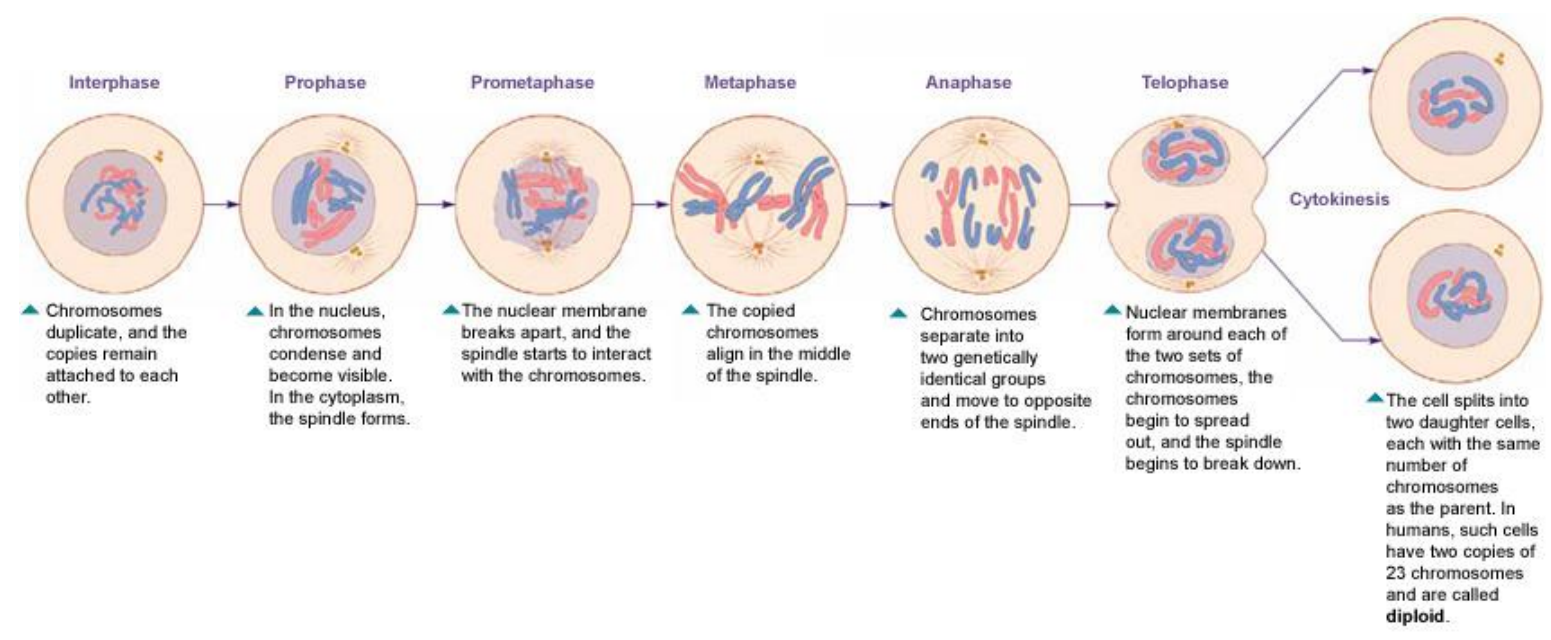

Figure 1-2. Schematic of the structural changes associated with the six stages of mitosis. First, the chromosomes are duplicated to form two identical copies. Next, the nuclear envelope degrades, and spindle fibers attach to the chromosomes and align them in the middle of the cell. The chromosomes are then separated into two identical groups and pulled to opposite sides of the cell. Finally, new nuclear envelopes form around the two chromosome clusters. After mitosis, the cell undergoes cytokinesis. (Adapted from the National Institute of General Medical Sciences [15]).

\subsubsection{Radiation Therapy}

Radiation therapy, or radiotherapy, uses ionizing radiation such as x-rays, gamma rays, or charged particles to kill pathological cells, and its use in cancer treatment traces back to the $19^{\text {th }}$ century. When x-rays were discovered in 1895 by German scientist Wilhelm Röntgen, they were initially explored for use in diagnostic imaging and little was known of their effects on biological tissue. By 1896, however, it was discovered that fluoroscopy experiments were causing biological effects such as epilation and dermatitis in subjects [16]. Though many experts believed the side effects of fluoroscopy were caused by electric discharge from x-ray tubes, Leopold Freund postulated that the x-rays themselves were 
responsible [16]. That same year, an American medical student named Emil Grubbé attempted to treat a breast tumor with an X-ray tube [17]. Since then, radiotherapy has become a staple in many cancer treatment regimens, due largely to significant scientific and technological advances that have made it both safer and more effective.

Radiotherapy kills cancer cells by damaging their DNA, either through direct or indirect actions (see Figure 1-3). Direct actions of radiation occur when the radiation interacts with DNA, causing breaks within its strands. Indirect actions of radiation occur when the radiation ionizes other molecules in the cell, such as water molecules, creating ions that in turn interact with DNA and cause breaks within its strands. The damaged strands can then be repaired by DNA polymerase, a protein that synthesizes DNA molecules, or the cell could be left with permanent irreparable damage that often leads to cell death. Since cancer cells often have faulty DNA repair mechanisms, they are more likely to experience irreparable DNA strand breaks and are therefore more likely than healthy cells to die when treated with radiation.

Radiation therapy is capable of localized geometric targeting at depths ranging from the surface to deep within the body, making it an ideal treatment for inoperable tumors. 


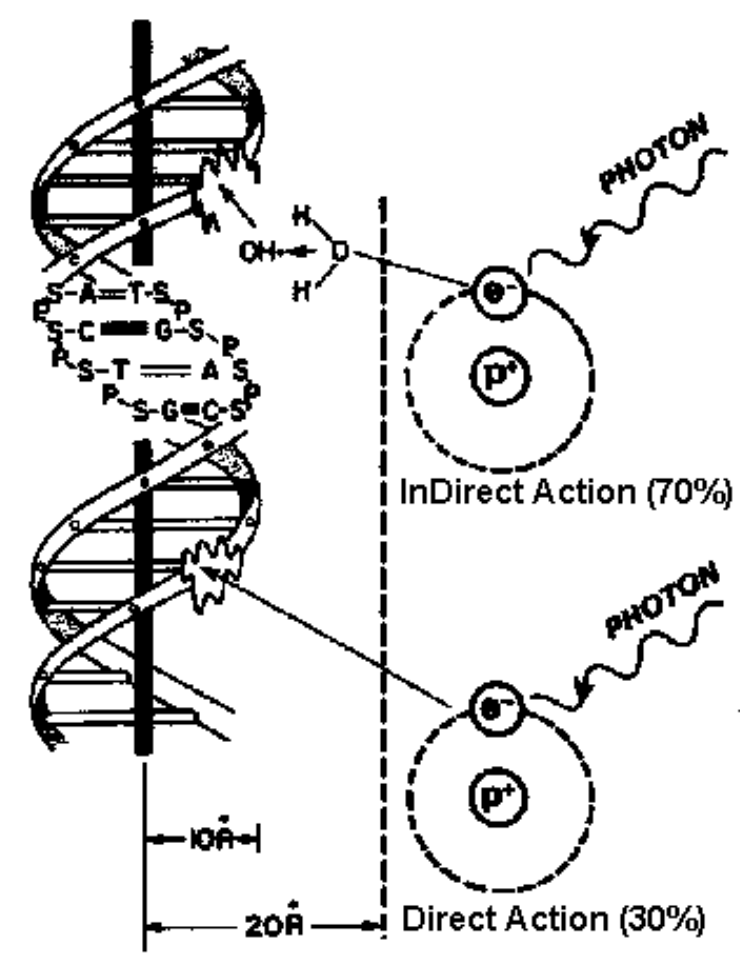

Figure 1-3. Direct and indirect methods of radiation damage. Indirect action accounts for approximately $70 \%$ of DNA damage in radiation therapy. (Adapted from Nias [18]).

\subsubsection{Chemotherapy}

Chemotherapy is a treatment that uses anti-cancer drugs, known as chemotherapeutic agents, to kill cancer cells. The history of chemotherapy traces back to the German introduction of chemical warfare during World War I, where highly devastating cytotoxic agents like mustard gas were used as weapons. Though chemical warfare was banned shortly after the conflict, the United States Department of Defense wanted to investigate possible therapeutic applications of mustard gas and other chemical warfare agents. Two American pharmacologists, Louis Goodman and Alfred Gilman, observed that mustard gas was too 
unstable to use in laboratory experiments and developed from it a more stable compound called nitrogen mustard [19]. They then carried out experiments in which they transplanted lymphoid tumors onto mice, treated them with nitrogen mustard, and observed that the tumors regressed after treatment [20]. Their colleague, a surgeon named Gustaf Lindskog, tested this treatment on human patients with Non-Hodgkin's lymphoma in 1943 and also saw significant tumor regression [20].

Chemotherapy has evolved drastically in the decades since its first use due to scientific advancements and discovery of more chemotherapeutic agents. Today, there are over three dozen unique chemotherapeutic agents used in cancer treatment regimens, though they can be classified into 5 main categories: (1) alkylating agents, (2) antimetabolites, (3) antimicrotubule agents, (4) antitumor antibiotics, and (5) topoisomerase inhibitors. Since cancer cells are generally metabolically active and undergo more frequent cell division than normal cells, many chemotherapeutic agents preferentially target cells undergoing or preparing for mitosis.

Alkylating agents are the oldest group of chemotherapeutic drugs in use today and include the aforementioned nitrogen mustard. They contain alkyl groups, which are chemical substituents that can covalently bind to proteins, DNA, and ribonucleic acid (RNA). When an alkyl group binds twice to a strand of DNA (called an intrastrand crosslink) or binds two strands of DNA together (called an interstrand crosslink), the DNA strands can break during replication. If the cell can't repair the DNA damage, it will eventually undergo apoptosis.

Antimetabolites attack cancer cells by preventing them from synthesizing DNA or RNA. They are structurally similar to naturally occurring metabolites that are involved in DNA/RNA synthesis and can compete with them to bind to key enzymes [21]. When DNA 
synthesis is impeded, a cell cannot successfully perform mitosis, eventually leading to cell death. Unlike alkylating agents, antimetabolites are cell cycle dependent as they only work when the cell's DNA is being replicated, a period in the cell cycle known as the S-phase (see Figure 1-4).

Anti-microtubule agents, or tubulin-binding drugs, bind to $\alpha$-tubulin or $\beta$-tubulin, proteins that polymerize to form microtubules. Microtubules are major components of the eukaryotic cytoskeleton and are necessary in mitosis, cellular migration, intracellular transport, angiogenesis, and other cellular functions [22]. Anti-microtubule agents can work either by preventing the formation of microtubules, inhibiting mitosis, or by preventing the disassembly of microtubules, inhibiting normal cell function [21].

Antitumor antibiotics, also called cytotoxic antibiotics, insert molecules into specific sites on DNA, inhibiting proper function and forming free radicals that cause strand breakages [21]. Though different antibiotics have varying mechanisms of action, the common theme they share is that they interrupt cell division to induce cell death.

Topoisomerase inhibitors are drugs that affect the activity of the enzymes topoisomerase I and topoisomerase II, which are responsible for uncoiling DNA during replication or translation. Inhibition of one or both enzymes interferes with these processes and ultimately leads to cell death. 


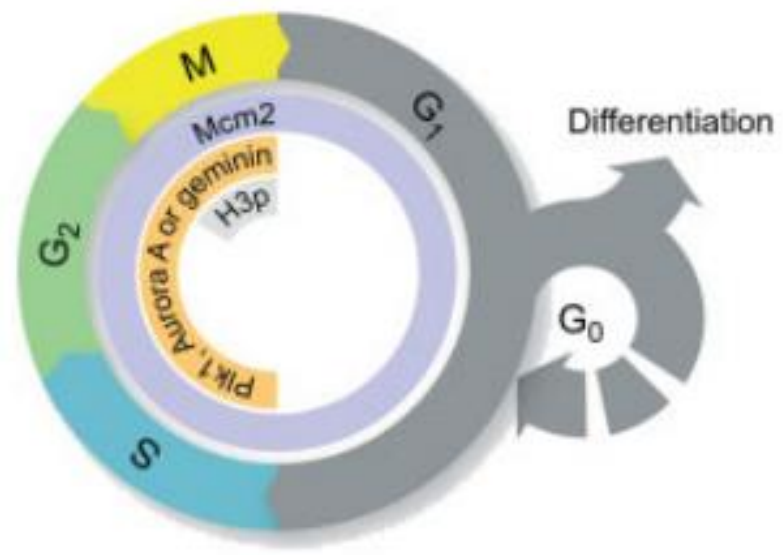

Figure 1-4. The cell cycle. Though most healthy human cells are not dividing and exist in "out of cycle" states (G0 phase), some cell types such as bone marrow, skin, and cancer, are always proliferating. During the G1 phase, cells grow. The $S$ phase is when DNA is replicated so that all chromosomes are doubled to form two pairs of sister chromatids. The cell continues to grow during the G2 phase, and mitotic spindles start to form. The M phase is classified by mitosis and cytokinesis. There are a number of biomarkers that can indicate which phase a cell is currently in, including Mcm2, Plk 1, Aurora A, geminin, and H3p. (Adapted from Williams and Stoeber [23]).

Compared to surgery and radiation therapy, chemotherapy has a distinct advantage in that it is a systemic treatment: most chemotherapeutic drugs are injected intravenously or ingested orally and can affect cancer cells all throughout the body, albeit with some limitations. 


\subsection{Cancer Treatment Monitoring}

Cancers are genetically diverse, and treatments can affect patients differently. Furthermore, tumors can develop resistance or immunity, rendering treatments less effective after repeated applications. Both chemotherapy and radiation therapy have side effects that impede a patient's quality of life, so, there is a need to regularly monitor the effectiveness of treatments to minimize patient discomfort and ensure effective clinical decision-making.

Current methods for monitoring cancer treatment are based on the Response Evaluation Criteria in Solid Tumors (RECIST), developed in 2000 [24] and later updated in version 1.1 [25]. First, images of a patient's tumors are acquired using a medical imaging modality such as x-ray imaging, ultrasound (US), or endoscopy, though this step is not necessary if all tumors are located on the surface of the skin. The largest lesions are then selected, and the sum of the largest diameters of all selected lesions (SAD) is computed as a baseline size measurement (see Figure 1-5). After treatment, the SAD is measured again, and the treatment response is evaluated based on this second measurement. A full response is defined by either a complete absence of lesions after treatment or a SAD of less than $10 \mathrm{~mm}$, a partial response is when the SAD has decreased by $30 \%$ or more, and disease progression is defined by a SAD increase of $20 \%$ or more.

The frequency of tumor re-evaluation is treatment- and patient-specific, though it is standard for patients to undergo baseline evaluation and then go through an entire round of treatment before their follow-up evaluation. This process can take 4 weeks or more, with a typical time frame of approximately 6-8 weeks between baseline evaluation and reevaluation [24]. The cost of administering an ineffective treatment can be very high both 
financially and in terms of lost quality of life for the patient resulting from treatment side effects. Furthermore, tumors can grow and metastasize in the time between treatment reevaluations, negatively impacting the patient's likelihood of survival. There is thus a desire for early treatment monitoring methods that can determine whether a cancer is responding within a few days of starting a treatment.

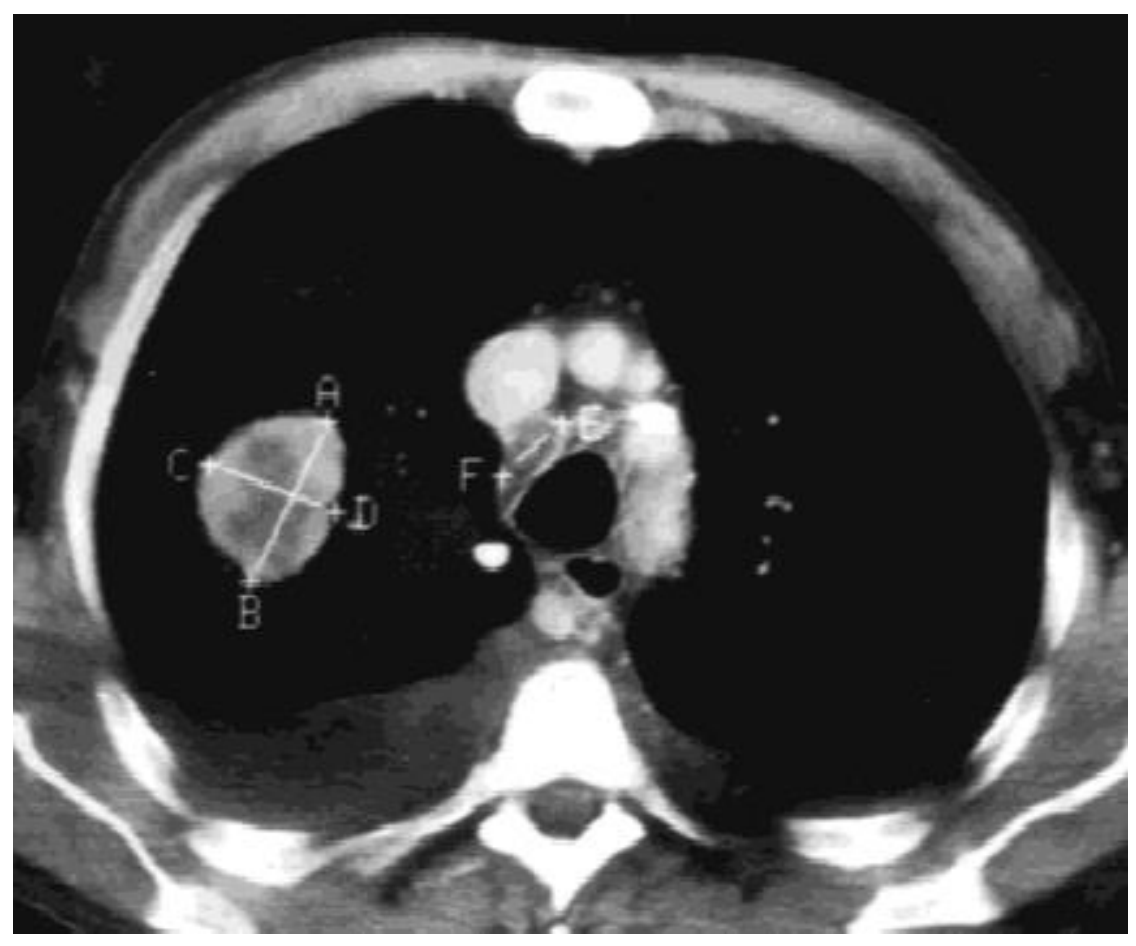

Figure 1-5. X-ray computed tomography (CT) image of a person's thoracic cavity in the transverse plane. Two cancerous lesions have been identified and measured with calipers. A large lesion is in the left lung and has a largest diameter shown by the A-B line, while a smaller lesion is located near the heart and has a largest diameter shown by the F-G line. RECIST only requires the largest diameter of each lesion, so the C-D line is not included in SAD calculations. (Adapted from Therasse et al [24]). 


\subsection{Early Treatment Monitoring}

Early treatment monitoring is based on the premise that tumors experience biological changes well before they undergo noticeable changes in size. Detectable tumor shrinkage only occurs after a significant number of cells within a tumor have died and been cleared out by immune cells, so in the early stages of treatment there is often little or no change in tumor size. Early treatment monitoring methods attempt to provide insights into the efficacy of treatments shortly after treatment initiation by monitoring cancer metabolism, vascular function, or cell death.

\subsubsection{Monitoring Cancer Metabolism}

Cancer cells generally have high energy demands due to their increased and unregulated proliferation. The primary energy source in eukaryotic cells that drives functions such as cell division and migration is ATP, and it's typically produced through processes that use glucose. In normal cells, ATP is generated primarily through oxidative phosphorylation, which is a process that yields approximately 36 ATP molecules per molecule of glucose but also requires oxygen. In many cancer cells, ATP is generated instead through aerobic glycolysis, which only yields approximately 4 ATP molecules per molecule of glucose but doesn't require oxygen [26]. The combination of high metabolic demands and an inefficient method of converting glucose to ATP leads cancer cells to consume a lot more glucose than regular cells, which can be detected with certain imaging modalities. Changes in the amount of glucose consumed by a tumor over time can indicate whether a treatment is inducing a response and can be monitored using positron emission tomography. 
Positron emission tomography (PET) is a biomedical imaging modality that can provide information about tumor metabolism. PET detects gamma rays that are emitted indirectly by a positron-emitting radionuclide, which is introduced into the human body by attaching it to a biologically active molecule. Fluorodeoxyglucose (FDG) is a radiolabeled positron-emitting molecule that is chemically analogous to glucose and accumulates in specific regions that consume a lot of glucose, such as in tumors and the brain, when injected into the bloodstream [27]. These highly metabolic sites show up as bright areas in a PET image. If a cancer treatment is effective, a significant portion of the tumor cells will have died, and the overall amount of glucose consumed by the tumors will decrease, which will manifest in the PET image. PET can also detect if new metastases form.

As in PET, single photon emission computed tomography (SPECT) involves administering radioisotopes to the patient and generating images by detecting the emitted radiation. Instead of positron emitters, SPECT uses gamma ray emitters such as 99mTechnetium and 131-Iodine. Studies have found a direct correlation between amino acid uptake and glucose metabolism in brain tumors, so SPECT imaging with radiolabeled amino acids has been proposed as an alternative to FDG-PET [28]. SPECT using iodine-123- $\alpha$ methyl tyrosine as a tracer was shown to be just as effective as FDG-PET in evaluating primary brain tumors [28].

A major limitation of PET and SPECT in terms of their potential for early treatment monitoring is that they use harmful ionizing radiation. To minimize patient exposure and reduce the chance of causing side effects, PET/SPECT imaging frequency must be limited.

Nuclear magnetic resonance spectroscopy (NMR), also known as magnetic resonance imaging (MRI), is another imaging technique capable of providing functional information 
about tumor metabolism. Standard NMR generates signals from the nuclei of hydrogen atoms, which are highly abundant in the human body, but it is also possible to generate signals from less abundant atoms like carbon-13 (13-C) [29]. When 13-C enriched glucose is administered to a patient, NMR later shows high concentrations of the molecule in organs with high glucose uptake, such as the brain [30]. Though this technique does not use ionizing radiation, it has poor sensitivity and low spatial resolution due to the relatively low abundance of 13-C molecules in the human body [29].

\subsubsection{Monitoring Hypoxia}

Hypoxia is a condition in which a region of the body is deprived of adequate oxygen supply. When tumors or sections of tumors are hypoxic, they do not respond as well to radiation therapy or chemotherapy, as oxygen plays a role in indirect DNA damage by radiation, and blood perfusion into a tumor is needed for chemotherapeutic agents to reach the cancer cells. Furthermore, tumor oxygenation levels have been linked to treatment effectiveness, as hypoxia in tumors post-treatment is indicative of treatment failure [31]. Thus, methods to monitor hypoxia in tumors during treatment can provide an indication of tumor response to treatment.

Hypoxia can be assessed indirectly by monitoring vascular function, since blood perfusion is required for a tumor to receive oxygen. High-resolution biomedical imaging modalities such as NMR, CT, and US can image blood flow with the use of contrast agents such as iron nanoparticles [32], iodine or gadolinium [33], or microbubbles [34], respectively. When these contrast agents are injected into the bloodstream, they either perfuse into areas of the tumor, indicative of adequate blood supply, or they remain out of areas of the tumor, 
indicative of hypoxia. Doppler US is another method that can measure blood flow speed and direction, albeit without the need for contrast agents [35].

\subsubsection{Monitoring Cell Death}

The main goal of cancer treatment is to kill cancer cells, and techniques that attempt to monitor cancer metabolism or hypoxia are only indirect assessments of cell death. Though hypoxia is correlated with poor treatment response, adequate tumor oxygenation does not necessarily indicate a good response as it provides no measure of cell death. Attempts to test treatment effectiveness by monitoring glucose metabolism are also imperfect, as it has been proven that some tumors can utilize ketone bodies instead of glucose as fuel, especially in conditions of glucose-deprivation [36]. Treatment monitoring methods that look at direct indicators of cell death are likely the best predictors of cancer treatment outcome as they assess tumor cell viability.

Cell death can be monitored by observing the predictable morphological and biochemical changes that occur in cells undergoing apoptosis. Since programmed cell death is generally repressed in cancer cells, it is rare for untreated or non-responding cancer cells to experience apoptosis [2]. Monitoring apoptosis can therefore be an effective way to monitor cancer treatment overall.

A cell undergoing apoptosis uses protein signals such as phosphatidylserine (PS) to attract macrophages, which then phagocytize the apoptotic bodies produced during the process. Within 90-120 minutes of the initiation of apoptosis, and before membrane blebbing occurs, PS is externalized on the outer surface of the cell membrane [37]. Annexin V, a human protein found in the cytoplasm of many cells, has a high affinity for cells with exposed 
PS and can be labeled with the radioactive tracer $99 \mathrm{~m}$-Technetium to form 99m-Tc-Annexin $\mathrm{V}$ and imaged with SPECT. In a study on 13 cancer patients, none of the patients showed uptake of 99m-Tc-Annexin V uptake in their tumors before chemotherapy treatment, but 7 showed increased uptake in as little as 4 hours after treatment and this correlated with a partial or complete response to treatment [37].

Quantitative ultrasound (QUS) is an US-based imaging modality in which backscatter intensity and spectral parameters are extracted from raw ultrasound signals and can be related to physical features of the imaged sample. QUS has been used to monitor the responses of cancer cells to chemotherapeutic treatments in vitro by detecting apoptosis [38]. Specifically, apoptosis was shown to be linked to a significant increase in ultrasound backscatter. More recently, it has been shown that an increase in cellular size variance, which can result from cell death processes like apoptosis and oncosis, correlates with an increase in ultrasound backscatter intensity, which could explain the previous results [39]. QUS has also been demonstrated effective at monitoring the response of breast cancer to treatment in vivo with cross-validated accuracies ranging from $82-86 \%$ [40]. 


\subsection{Detecting Cell Death with Optical Coherence Tomography}

Optical coherence tomography (OCT) is an imaging modality that uses backscattered light to generate cross-sectional sagittal plane images that are analogous to US B-mode images. Compared to commonly used clinical imaging modalities, such as US and MRI, OCT has very high spatial resolution on the order of microns. However, OCT has relatively low imaging penetration compared to other modalities due to the high optical absorption of biological tissues (see Figure 1-6).

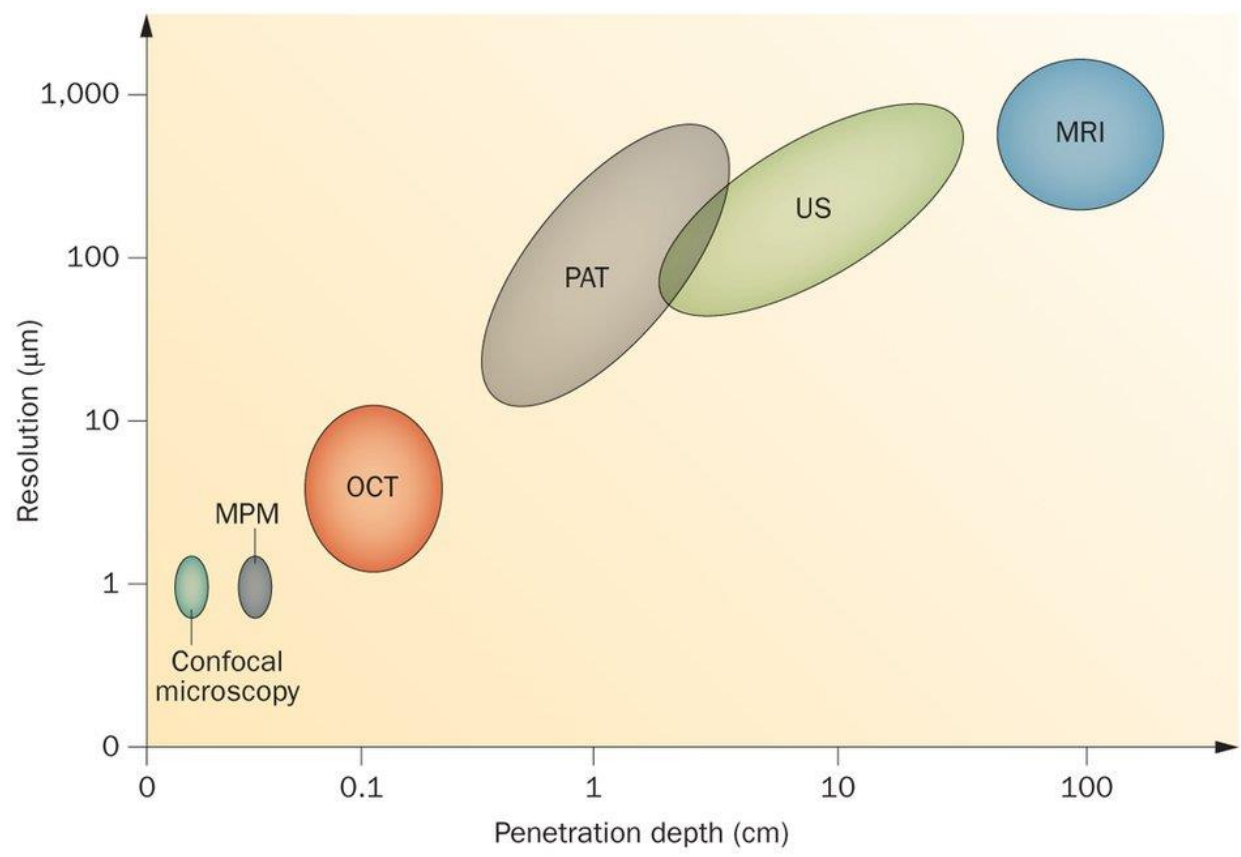

Figure 1-6. Resolution and penetration of various imaging modalities. Confocal microscopy and multiphoton microscopy (MPM) have very high spatial resolution but low penetration depth compared to OCT. Photoacoustic tomography (PAT), US, and MRI have better penetration than OCT but lower spatial resolution. (Adapted from UBC Biomedical Engineering [41]). 
Previously, Farhat et al demonstrated that OCT could be used to detect cell death in acute myeloid leukemia (AML) cancer cell populations in vitro [42], [43]. The aim of this thesis, discussed in detail in Section 1.6, is to further develop some of the methods used in those studies.

This chapter summarizes the principles of OCT, the techniques used to detect cell death with OCT, and the relevant previous study that is a precursor to this work.

\subsubsection{Principles of OCT}

The most common configuration used in OCT is based on the Michelson interferometer (see Figure 1-7). A beam of light from a laser source is split into two distinct paths known as the reference arm and the sample arm, respectively. The reference arm leads to a mirror that reflects light back to the detector while the sample arm leads to the sample that is being imaged, where some of the light is backscattered to the detector while the rest is either absorbed or scattered off in other directions. If there is complete or partial coherence between the beams from the two arms, they recombine and interfere at the detector.

Coherence can be envisioned as the degree of correlations that exist between the light fluctuations of two interfering beams [44], and two overlapping waves of light are said to be perfectly coherent if they have a constant phase difference, frequency and waveform. In OCT, the degree of coherence between light from the reference arm and light from the sample arm depends on the reference arm path length: light that is reflected by the mirror is most coherent with light from the sample that has traveled the same distance. The resulting

interference signal therefore carries information about sample reflectivity only within a specific depth range in the sample. 


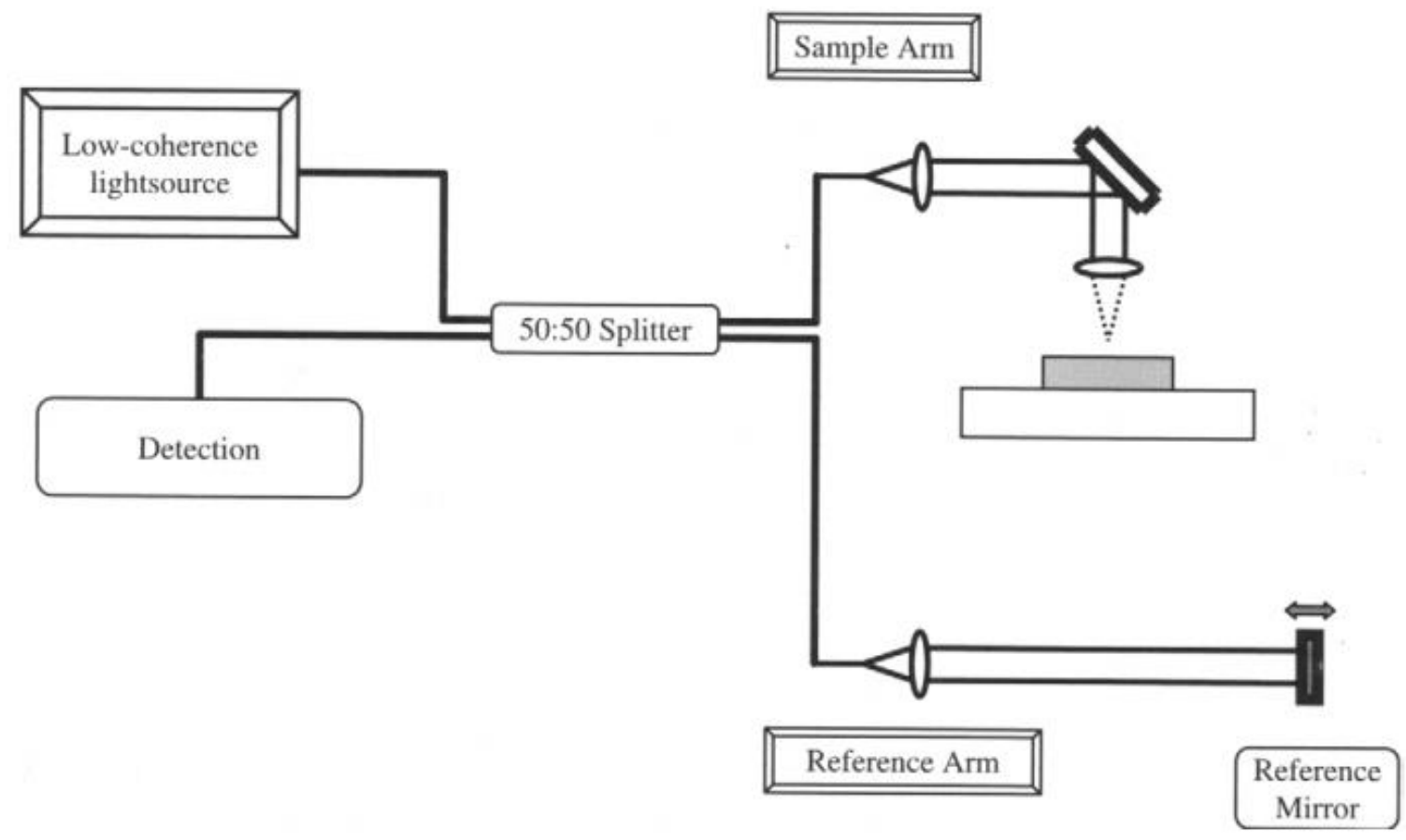

Figure 1-7. Simplified schematic of an OCT instrument based on the Michelson interferometer. Light from the source is split into two paths: one to a reference mirror and the other to the sample. Then, the light reflected by the mirror recombines with the light backscattered by the sample, creating an interference signal. (Adapted from Brezinski [44]).

Axial resolution in OCT is equal to the round-trip coherence length, which is the propagated distance over which light from the source maintains its coherence. The coherence length is related to the central wavelength and bandwidth of the light source, and is given by [44]:

$$
L_{c}=\frac{2(\ln 2) \lambda_{0}{ }^{2}}{\pi \Delta_{\lambda}}
$$


where $L_{c}$ is the coherence length or axial resolution, $\lambda_{0}$ is the central wavelength of the light source, and $\Delta_{\lambda}$ is the full width at half maximum bandwidth of the light source.

OCT lateral resolution is dependent on the objective lens used to focus light, and is given by:

$$
\Delta x=\frac{4 \lambda_{0} f}{\pi \mathrm{d}}
$$

where $\Delta x$ is the lateral resolution, $\lambda_{0}$ is the central wavelength of the light source, $f$ is the focal length of the objective lens, and $d$ is the focal spot width of the objective lens. Lateral and axial resolutions are decoupled in OCT, therefore high depth resolution can be achieved even in the absence of high numerical aperture (NA) lenses, and high lateral resolution can be achieved in the absence of a broad bandwidth source.

There are multiple methods to acquire depth information in OCT: earlier systems used a method known as time domain OCT (TD-OCT) to acquire interference signals at different depths whereas systems today typically use spectral domain OCT (SD-OCT) methods. In TD-OCT, the length of the reference path is manipulated by moving the reference mirror, allowing acquisition of interference signals from varying path lengths [44]. When the reference arm is shortened, the interference signal comes from light backscattered closer to the surface of the sample, whereas when the reference arm is lengthened, the interference signal comes from light backscattered deeper within the sample. To produce a full sagittal cross-sectional image in TD-OCT, many signals from different depths have to be acquired at each position in the sample, and either the sample arm or the sample itself has to be shifted 
laterally to acquire signals at different transverse positions, which limits imaging speed. Furthermore, the movement of the reference mirror creates motion noise.

In SD-OCT methods, the reference mirror does not move, so the reference arm has a fixed path length throughout the entire imaging process. Depth information is acquired in the form of a spectral interferogram, which is a signal that relates interference intensity to frequency instead of relating intensity to depth. An image with depth information can be formed using the Fourier relationship between depth and frequency [45]:

$$
F_{S}(z) \propto F T\left\{A_{S}(k)\right\}
$$

where $F_{S}(z)$ is the reflectivity profile as a function of depth, and $A_{S}(k)$ is the backscattered amplitude as a function of frequency, also called the source spectrum. The reflectivity profile at a lateral position in the sample depicts the intensity of light backscattered by the sample and captured by the OCT device convolved with the system point spread function. It is expressed as a function of sample depth, and is estimated as the inverse Fourier transform of the source spectrum. Typical OCT reflectivity profiles depict a decay in signal with increasing depth due to light attenuation within the sample.

\subsubsection{Dynamic Light Scattering}

Dynamic light scattering (DLS) is a technique that involves analyzing the temporal fluctuations that occur in the light-scattering profile of a sample by means of intensity or photon autocorrelation function (ACF) analysis. DLS can be used to acquire information about the size, speed, and shape of scatterers based on temporal changes in their light 
scattering profile [46], and is commonly used to determine the size of particles in a solution by studying their Brownian motion [47].

A typical setup for DLS is shown in Figure 1-8. A laser directs a beam of light onto the sample, where some of it is absorbed and the rest is scattered. A photodetector converts the scattered light into an intensity time-signal that is input into a correlator that computes the signal ACF. An ACF is a measure of the correlation between a signal and a delayed copy of itself as a function of the delay, and in DLS the ACF typically has a maximum value when time lag is zero, and decays with increasing time lag to a minimum value. For monodisperse spherical particles experiencing Brownian motion in a solution, the ACF can be represented by an exponential decay function given by [48]:

$$
g(\tau)=C e^{-\frac{-32 \pi^{2} D}{\lambda^{2}} \sin ^{2}\left(\frac{\Theta}{2}\right) \tau}
$$

where $\tau$ is the time lag, $g(\tau)$ is the ACF as a function of time lag, $\lambda$ is the wavelength of light in the medium, $C$ is a constant related to the total amount of light backscattered across all frames, $\Theta$ is the scattering angle and $D$ is the diffusion coefficient of the particles given by [48]:

$$
D=\frac{k_{B} T}{6 \pi \eta a}
$$

where $k_{B}$ is Boltzmann's constant, $T$ is the absolute temperature of the solution, $\eta$ is the viscosity of the solvent, and $a$ is the radius of the particles in the solution. Equation (5) is also known as the Einstein-Stokes relationship. 


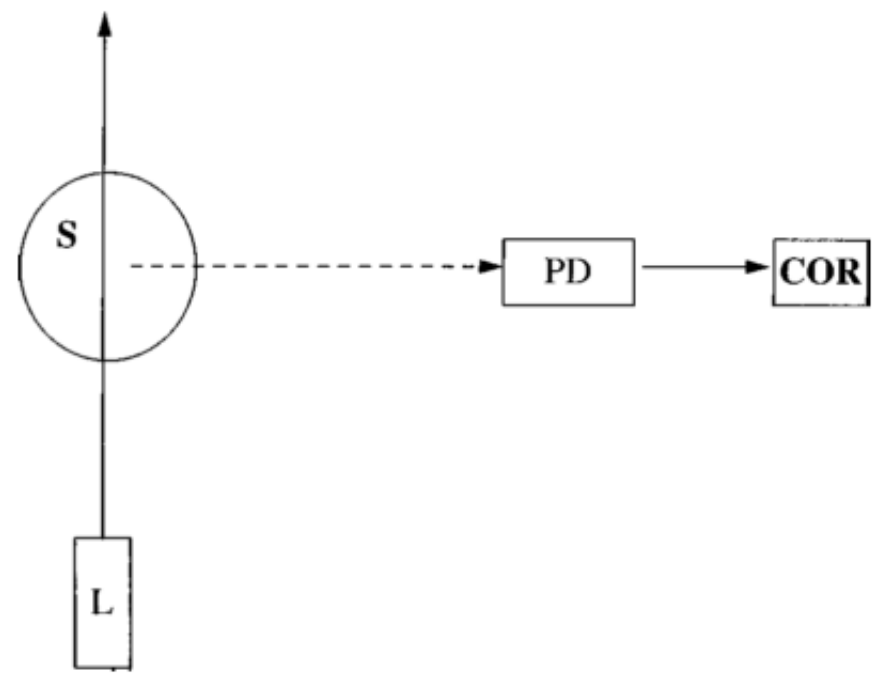

Figure 1-8. Schematic representation of a typical DLS setup. The source $(L)$ illuminates the sample $(S)$ with light, which is scattered. Some of the scattered light is captured by a photodetector (PD) which then inputs the signal to a correlator (COR). (Adapted from Goldburg [46]).

The exponential decay of an ACF can be expressed in terms of the decorrelation time, also known as the relaxation time, which is the time for an exponential variable to decay from a maximum value to $1 / e$ of that maximum value:

$$
g(\tau)=C e^{\frac{-\tau}{T_{C}}}
$$

where $T_{C}$ is the decorrelation time. The decorrelation time can be thought of as a variable that directly describes how rapidly an ACF decays; a large decorrelation time indicates a slowly decaying exponential function, while a small decorrelation time represents a rapidly 
decaying function. Comparing Equations (4), (5), and (6) reveals that the decorrelation time of an ACF from a solution of monodisperse spheres is inversely related to the diffusion coefficient of the spheres and directly related to their radii. Thus, if all other variables are known, DLS techniques can be used to determine the mean size or diffusion coefficient of particles in a monodisperse solution.

In the case of a polydisperse solution, the ACF produced with DLS techniques no longer resembles a single exponential decay function, and can instead be represented by a sum or distribution of exponentials with a broad distribution of decorrelation times [49], given by:

$$
g(\tau)=\int_{0}^{\infty} G\left(T_{C}\right) e^{\frac{-\tau}{T_{C}}} d T_{C}
$$

where $G\left(T_{C}\right)$ is the distribution function of the decorrelation times. The objective of data analysis of an ACF from a polydisperse solution is often to characterize $G\left(T_{C}\right)$ to provide information about the size distribution of particles in the solution.

\subsubsection{Decorrelation Time Distribution Analysis}

One of the methods commonly used in DLS to obtain information about the size distribution of polydisperse particles in a solution is known as the cumulants method, first introduced by Koppel [49] in 1972. In probability theory, cumulants are a set of quantities that provide information about a distribution. In DLS, the first three cumulants of an ACF are given by [50]: 


$$
\begin{gathered}
\kappa_{1}=\int_{0}^{\infty} G\left(T_{C}\right) T_{C} d T_{C}=\overline{T_{C}} \\
\kappa_{2}=\mu_{2} \\
\kappa_{3}=\mu_{3}
\end{gathered}
$$

where $\kappa_{i}$ is the $i^{\text {th }}$ cumulant, $\overline{T_{C}}$ is the mean decorrelation time of the distribution, and $\mu_{i}$ is the $i^{\text {th }}$ moment about the mean, defined as:

$$
\mu_{m}=\int_{0}^{\infty} G\left(T_{C}\right)\left(T_{C}-\overline{T_{C}}\right)^{m} d T_{C}
$$

The second cumulant is the variance of the distribution and provides an indication of the polydispersity of the solution. A solution can be said to be monodisperse if the second cumulant is close to zero, and polydisperse otherwise. The third cumulant provides information about the skewness or asymmetry of the decorrelation time distribution [50].

The basis of cumulant analysis lies in expanding the logarithm of $g(\tau)$ as follows [50]:

$$
\ln [g(\tau)]=-T_{C} \tau+\frac{\kappa_{2}}{2 !} \tau^{2}+\frac{\kappa_{3}}{3 !} \tau^{3} \ldots
$$

Least squares methods can be used to fit the ACF data from DLS measurements to Equation 10 to solve for the first three cumulants of the distribution.

Though cumulant analysis provides information about the polydispersity of a solution, it only provides a mean decorrelation time. The CONTIN algorithm, developed by Stephen 
Provencher in 1981 [51] and first implemented in the Fortran programming language [52], can be used to distinguish between different particle populations.

CONTIN is a constrained regularization method used in DLS to determine $G\left(T_{C}\right)$, the distribution function of decorrelation times, from an ACF. In mathematics, regularization is a process in which additional information is introduced in order to solve an ill-posed problem, which is a problem that either has no solution, has multiple possible solutions, or does not continuously depend on the initial conditions. By making use of a regularization parameter, CONTIN produces a unique solution to the distribution function of an ACF for that particular regularization parameter.

The regularization parameter in CONTIN, denoted $\alpha$ and also referred to as the regularizer, is a number greater than or equal to 0 that is entered into the algorithm as an input by the user. The regularizer controls the preferred level of "spikiness" in the distribution, with smaller $\alpha$ values producing spikier distributions and larger $\alpha$ values producing smoother and less resolved distributions (see Figure 1-9). Choosing a regularizer that is too low can produce phantom peaks in the resulting distribution, which are peaks that do not represent physical phenomena and instead can be thought of as artifacts in the distribution. On the other hand, choosing a regularizer that is too high can blend true peaks together. There have been attempts in recent years to develop a method to determine the optimal regularizer to use in CONTIN for a specific set of data [53]. 


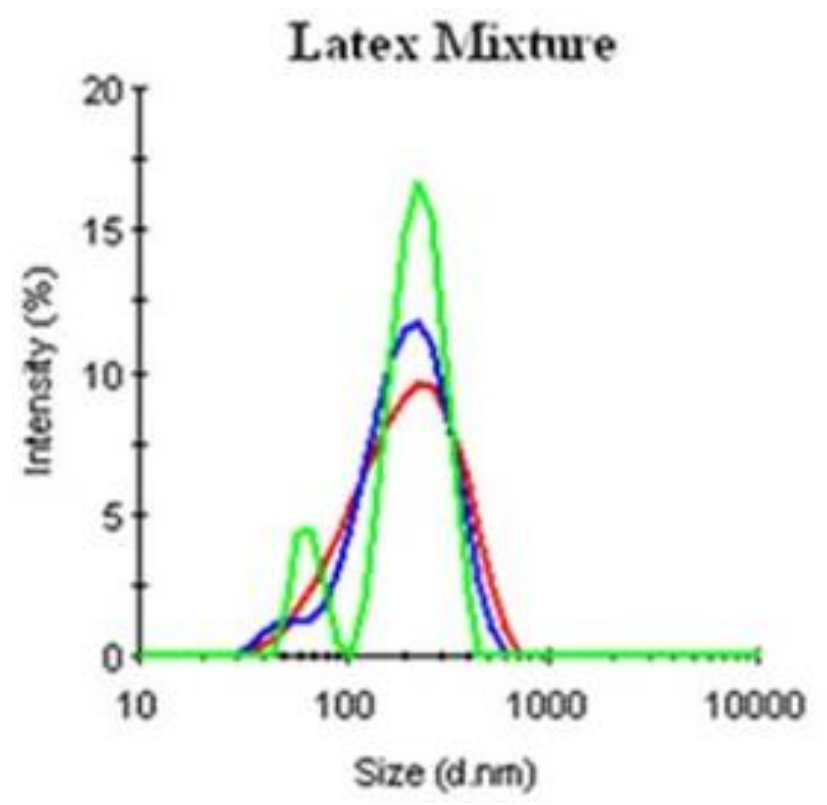

Figure 1-9. Size distributions of a latex solution generated using CONTIN. The actual solution contained 60 $\mathrm{nm}$ and $220 \mathrm{~nm}$ spheres. When a lower regularizer was used (green), CONTIN produced two distinct peaks for the $60 \mathrm{~nm}$ and $220 \mathrm{~nm}$ spheres. When higher regularizers were used (blue and red), the two populations were blended into a single peak. (Adapted from Malvern Panalytical [54]).

CONTIN also uses other a priori data to produce an optimal distribution for an ACF. The user inputs the range of all possible decorrelation times that act as limits on the distribution. Optionally, the user can specify the number of peaks in the distribution if it is known. A detailed description of the CONTIN algorithm and how it works can be found in the two original papers by Provencher [51], [52]. In more recent years, the algorithm was implemented in Matlab by Iari-Gabriel Marino [55]. 


\subsubsection{Dynamic Light Scattering Optical Coherence Tomography}

DLS techniques have successfully been applied to OCT intensity-time signals, despite the differences between the standard DLS setup (Figure 1-8) and the OCT interferometric setup (Figure 1-7). The integration of DLS with OCT is called dynamic light scattering OCT (DLS-OCT). Since OCT only captures backscattered light, which is light that has been scattered $\sim 180^{\circ}$ from the sample, the sinusoid term in Equation (4) simplifies to a value of 1. Lee et al [56] derived a formula to describe the ACF produced in a resolution volume in DLS-OCT, which takes into consideration the finite size of an OCT resolution volume, the presence of static scatterers, and the different types of motion that a particle can experience:

$$
\begin{gathered}
g(\tau)=M_{S}+M_{F} e^{-h_{t}^{2} v_{t}^{2} \tau^{2}} e^{-h^{2} v_{Z}^{2} \tau^{2}} e^{-\frac{-32 \pi^{2}}{\lambda^{2}} D \tau} \\
+\left(1-M_{S}-M_{F}\right) \delta(\tau)
\end{gathered}
$$

where $g(\tau)$ is the magnitude of the ACF normalized to a maximum value of $1, h_{t}$ is the inverse of $1 / \mathrm{e}$ of the transverse resolution, $h$ is the inverse of $1 / \mathrm{e}$ of the axial resolution, $v_{t}$ is the transverse speed of moving scatterers, $v_{z}$ is the axial speed of moving scatterers, $M_{S}$ is the intensity contribution from static scatterers, $M_{F}$ is the intensity contribution from the moving scatterers, and $\left(1-M_{S}-M_{F}\right)$ is the intensity contribution from particles entering or exiting the resolution volume and from noise or system defects. Static scatterers produce a backscattered intensity signal that does not fluctuate in time, so their contribution to the ACF is a constant value. Particles experiencing flow or diffusion contribute to an exponential 
decay in the ACF since their backscattering profile changes with time. Particles that enter or exit the resolution volume contribute to a Dirac delta function in the ACF. The different types of scattering are depicted in Figure 1-10Figure 1-10.

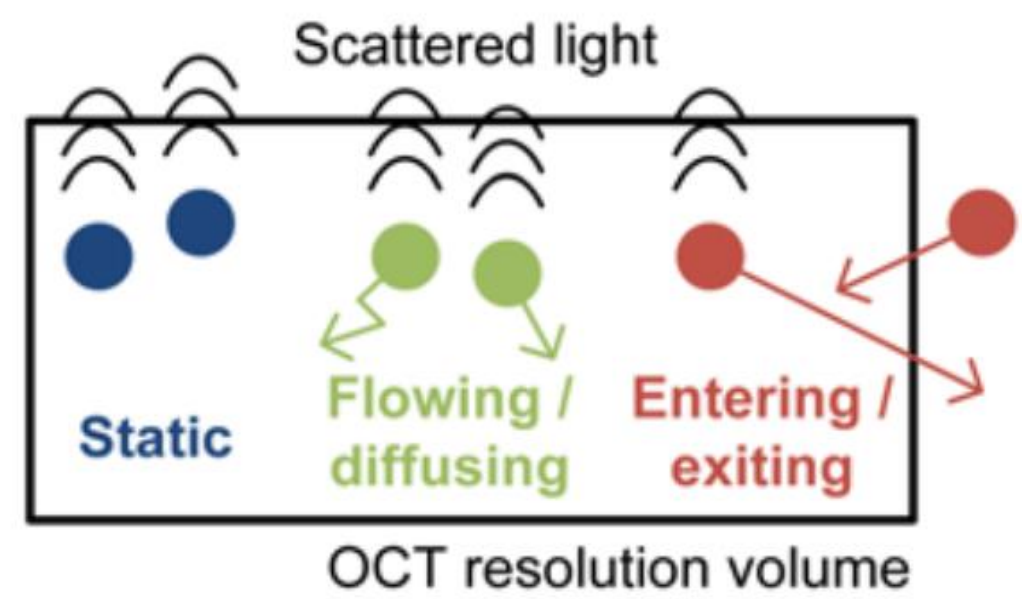

Figure 1-10. Conceptual illustration of OCT resolution volume and light scattering contributions to DLS-OCT theory. Particles within an OCT resolution volume can be categorized into three groups at any point in time: static, moving, or entering and exiting an imaging voxel. (Adapted from Lee et al [56]).

DLS-OCT has been demonstrated to be an effective tool for observing numerous biological phenomena such as imaging blood flow and intracellular motility in the brain [57], imaging extracellular matrix remodeling in vitro [58], and measuring the growth of biofilms [59]. In these biomedical applications and others, DLS-OCT examines the signal fluctuations 
from cells and subcellular components, whose light scattering properties are governed by the theory of light scattering by small particles.

\subsubsection{Light Scattering by Small Particles}

The classic text Light Scattering by Small Particles by H. C. van de Hulst [60] covers in detail the theory behind the light scattering properties of particles on the scale of cells and organelles. When an object is illuminated by light, charges within the atoms of the object oscillate as a result of interactions with the electric field of the incident electromagnetic wave. Some of the energy of the incident wave can be converted into thermal energy in a process known as absorption, while the rest is radiated from the object in a process called scattering. The light scattering and absorption properties of a medium are related to the degree of heterogeneity in the medium, which can be quantified by local changes in its optical refractive index (which is given by the ratio of the speed of light in air to the speed of light in the medium). A greater mismatch of refractive indices between different objects or materials results in more light scattering.

OCT interference signals result from the superposition of scattered photons from many different particles within a resolution volume. When many scatterers exist within a single resolution volume, the system can quickly become too complex to define mathematically as one would have to consider not only the light directly scattered by each particle after illumination from the source, but also collisions between particles and light that is scattered from particle to particle, known as multiple scattering. Thus, it is simpler to consider how a single particle scatters light to estimate the scattering profile. To further 
simplify the calculations, the particle is often assumed to be a homogeneous sphere, for which there exists an exact analytical solution to the scattering problem.

There are different models to describe how light is scattered by a spherical object, and the ratio between the size of the particle and the wavelength of light incident upon it determines which model fits best (see Figure 1-11). The two most extreme cases occur when the illuminated object has a diameter that is significantly smaller than the incident light wavelength, and when the object has a diameter that is significantly larger than the incident light wavelength. In the first case, a very small fraction of incident light is scattered, and most is transmitted through the object. In the latter case, light propagation can best be described by assuming that all light travels in a straight path and is reflected, transmitted, or refracted. 


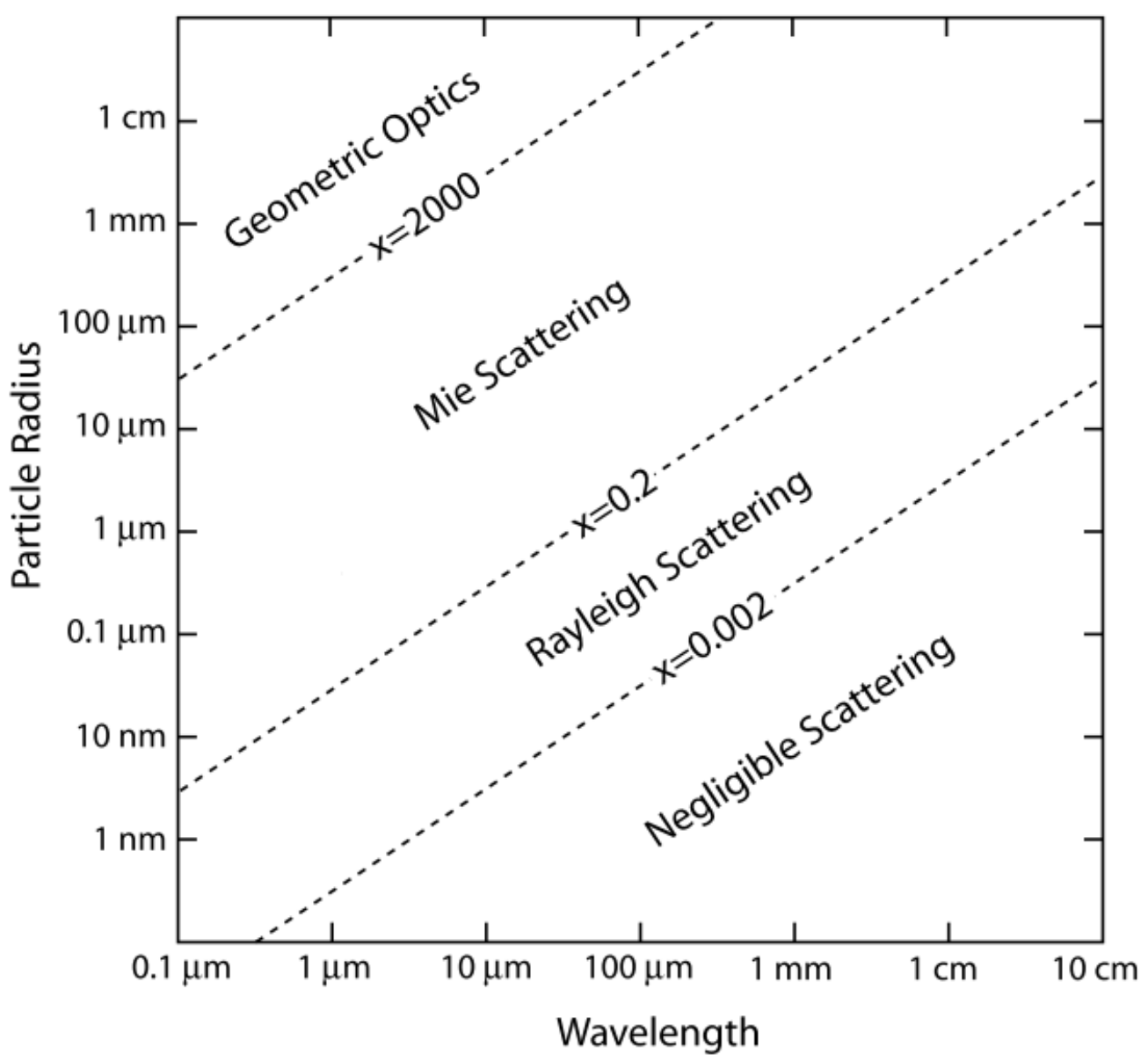

Figure 1-11. The different light scattering regimes as a function of particle size and incident light wavelength. The ratio $x$ is equal to $2 \pi r / \lambda$ where $r$ is the radius of the particle and $\lambda$ is the incident light wavelength. (Adapted from Petty [61]).

Rayleigh scattering is the elastic scattering of light by particles much smaller than the wavelength of incident light such that the particle's radius is approximately $0.001 / \pi$ to $0.1 / \pi$ 
times the wavelength. The intensity of light scattered by particles within the Rayleigh scattering regime is given by:

$$
I=\frac{I_{0} 8 \pi^{4} \alpha^{2}}{\lambda^{4} R^{2}}\left(1+\cos (\theta)^{2}\right)
$$

where $I$ is the scattered light intensity at a scattering angle $\theta, I_{0}$ is the incident light intensity, $\alpha$ is the molecular polarizability of the scatterer, $\lambda$ is the wavelength of the incident light, and $R$ is the distance between the light source and the particle. In the Rayleigh scattering regime, the intensity of forward-scattered light is approximately the same as the intensity of backscattered light and double the intensity of perpendicularly scattered light (see Figure $1-12)$.

The Mie scattering regime describes the scattering of light by a sphere of approximately the same size as or slightly larger than the incident light wavelength. The Mie solution takes the form of an infinite series, so computational methods have been used to approximate solutions instead. In general, Mie scattering predicts that most light is scattered in the forward direction (see Figure 1-12). 


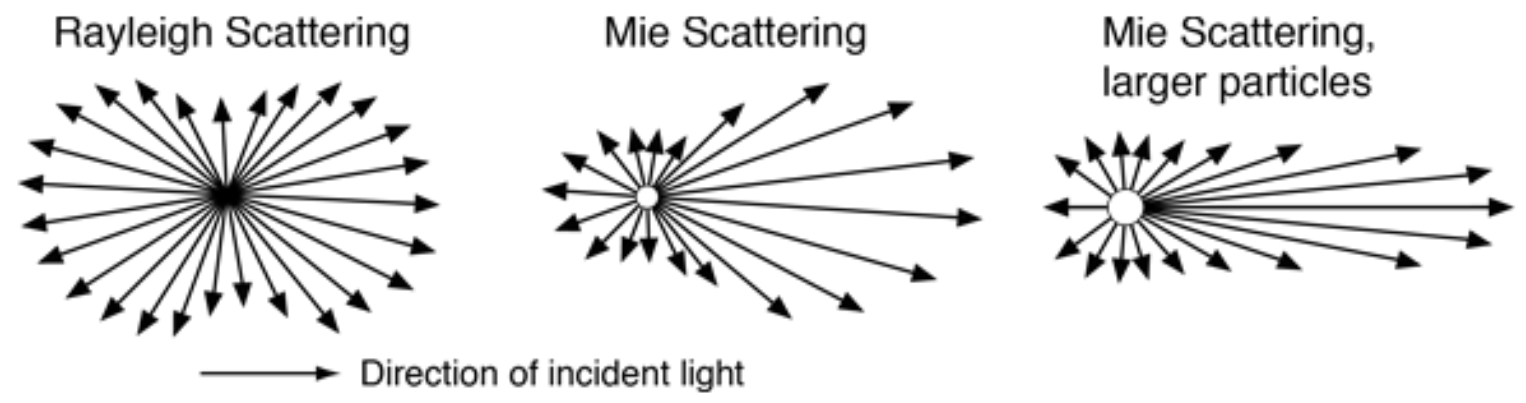

Figure 1-12. Scattering profiles of Rayleigh and Mie scattering regimes. Rayleigh scattering results in approximately the same intensity of light scattered in the forward direction and backward direction. In Mie scattering, however, significantly more light is scattered forward. (Adapted from [62]).

When using OCT to observe tissues, the most appropriate scattering regime can be determined by comparing the wavelength of the OCT laser source to the sizes of cells and their organelles. OCT systems typically use laser sources with wavelengths in the nearinfrared (NIR) range, which consists of wavelengths between 0.75-1.4 $\mu \mathrm{m}$. Eukaryotic cells are generally between $10-100 \mu \mathrm{m}$ in diameter [63]. Organelles can be even smaller than that, with mitochondria typically being approximately $1 \mu \mathrm{m}$ wide at their thinnest point [64]. For these size and wavelength ranges, Mie scattering offers the most accurate solution to the scattering problem. It should be noted, however, that scatterers in tissues are not always spherical, so solutions to the light scattering problem within cells are only approximations. 


\subsubsection{Detecting Cell Death with DLS-OCT}

The use of OCT to detect cancer cell death is based on the theory that cells experience changes in morphology and intracellular motion while undergoing cell death processes like apoptosis, and that these alterations are detectable with DLS techniques. Many of the changes that occur in apoptotic cells, including cellular and organelle fragmentation and dismantling of the cytoskeleton, contribute to an overall net increase in intracellular motion, which is hypothesized to manifest in DLS-OCT as a more rapidly-decaying ACF.

Farhat et al [43] were the first to use DLS-OCT to detect cell death in vitro. They treated acute myeloid leukemia (AML) cells with cisplatin, a chemotherapeutic agent that interferes with DNA replication to initiate apoptosis, and then centrifuged the cells into a tightly-packed pellet. OCT signals were acquired at different time points from 0 to 48 hours after treatment and ACFs were generated from specific regions of interest (ROIs). They processed the ACFs using the heterodyne method [65] to remove the static scattering contribution, and the decorrelation time was defined as the half width at half maximum of the modified ACF. They found that there was a significant drop in the decorrelation time 24 hours after the start of treatment, indicative of increased intracellular motion. The modified ACFs and decorrelation times from the study are shown in Figure 1-13. 

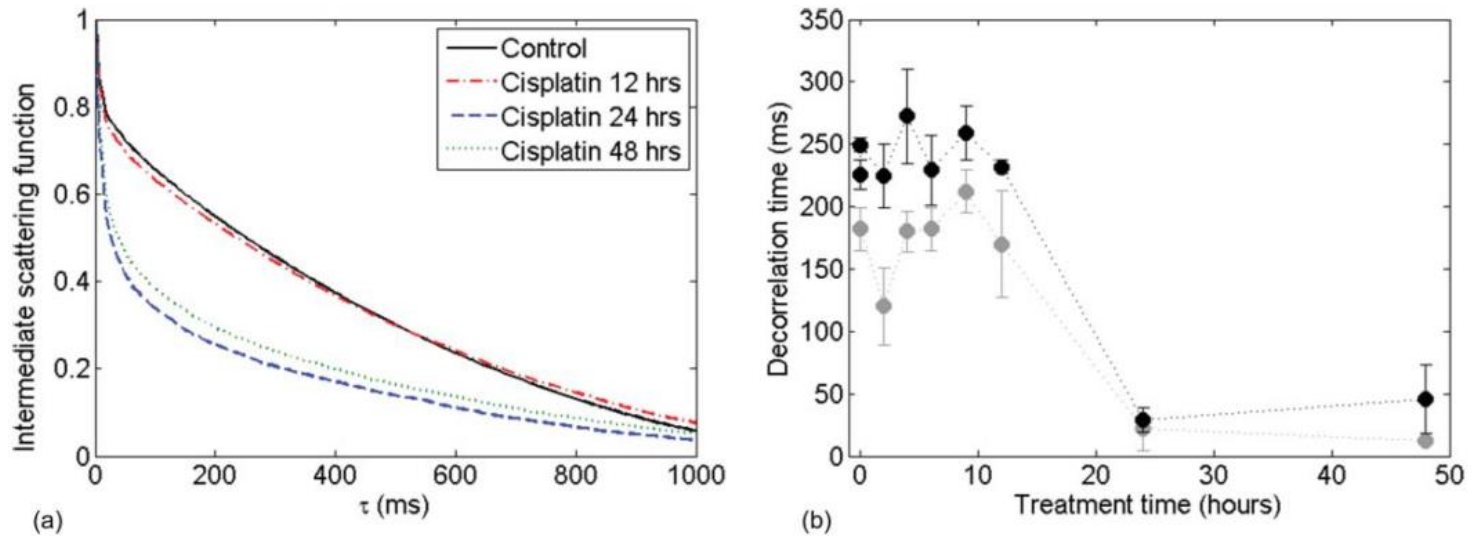

Figure 1-13. Results of DLS-OCT analysis of AML cells treated with cisplatin. (a): The intermediate scattering functions, which are the processed ACFs, from a control sample and three treated samples at different time points. (b): Decorrelation time as a function of treatment time. Error bars represent the standard deviation of 10 separate measurements from each sample. (Adapted from [43]).

AML cells proliferate rapidly and are not adherent, which are traits that make them suitable for research experiments that require the formation of in vitro cell pellets. Adherent cells require a surface to grow on, and attach to each other and the surface via plasma membrane proteins. As a result, they form monolayers and their growth is limited by the surface area of the flasks in which they are cultured. In contrast, non-adherent cells can be grown in suspension without being attached to any surface, which allows them to be grown to a higher density than adherent conditions would allow. The combination of rapid proliferation and non-adherence allows large AML cell populations to be cultured in short periods of time.

A major limitation of AML cells in in vivo research studies is that they very rarely form solid tumors [66], and instead typically flow freely in the bloodstream or are confined 
within the bone marrow of the affected person or animal. Thus, AML cells are not a good model for in vivo studies of solid tumors. 


\subsection{Motivation, Hypothesis and Specific Aims}

In this thesis, we describe a method to use DLS-OCT to detect death in breast cancer cells by observing changes in intracellular motion. We predict that the average speed of intracellular motion in apoptotic cells will be higher than in viable cells, even when using an adherent breast cancer cell line instead of a non-adherent blood cancer cell line, as was done in past studies. This is because all apoptotic cells undergo similar processes, and there are not significant differences in the key apoptotic morphological changes between different cell types. Furthermore, we hypothesize that cells experiencing oncosis because of nutrient deprivation will also exhibit higher rates of intracellular motion because oncotic cells swell with fluid, creating more space in the otherwise tightly packed cells for organelles such as mitochondria and lysosomes to diffuse.

Pixel intensity in an OCT image depends on the optical properties, distribution, and quantity of scatterers within a resolution volume. When there is movement within a resolution volume, pixel intensity changes as a function of time due to changes in the net light scattering profile. DLS techniques can be used to detect and quantify motion in consecutive OCT images by autocorrelation analysis of fluctuating pixel intensities. When OCT is used to image living cells in vitro, pixel intensity fluctuates primarily due to intracellular motion since there is no significant extracellular motion, such as blood flow. As such, DLS measurements can provide an indirect measure of the intracellular motion in in vitro cell samples.

DLS-OCT has been used to detect cell death in AML cells in vitro by quantifying changes in intracellular motion that occur during apoptosis [43], which is a common mode 
of cell death for cancer cells that have been treated with chemotherapy or radiotherapy. Apoptosis is characterized by predictable changes in cellular morphology such as rounding and shrinking of the cell, fragmentation of the organelles, and disintegration of the cell into membrane-bound apoptotic bodies. The net result of these changes is that there is an overall increase in intracellular motion.

Cell death by oncosis is also characterized by predictable morphological changes, such as swelling of the cell and its organelles followed by rupturing of the cell. Oncosis is the primary mode of cell death for cells that are depleted of ATP, which is an important factor in cancer treatments that attempt to deprive tumors of nutrients.

Light scattering within cells is caused by differences in the local refractive index between organelles and the cytosol, which is the liquid found inside cells and the medium in which the organelles are situated. Table 1-1 displays typical refractive indices of the cytosol and some of the most significant light scatterers within cells: the nucleus, mitochondria, and lysosomes. Nuclei are the largest organelles in cells, ranging from 2-10 $\mu \mathrm{m}$ in diameter and housing the cell's DNA. Though they do not have a large refractive index mismatch with cytosol, nuclei scatter a significant portion of the incident light due to their large size. Mitochondria are organelles with many functions, including the production of ATP for the cell, and they range in size drastically because they can link together to form chains via a process called mitochondrial fusion. While most animal cells typically only have one nucleus, they can have many mitochondria, though this depends on the cell type [64]. A single cell can have as little as zero mitochondria, as in the case of blood cells, or it can have hundreds, as in the case of hepatocytes. Finally, lysosomes are membrane-bound organelles 
found in animal cells that contain enzymes. Though lysosomes are small, ranging from 0.1$1.2 \mu \mathrm{m}$ in diameter [67], they have a significant refractive index mismatch with the cytosol.

Table 1-1. Typical refractive indices for various organelles in a cell. (Adapted from [68]).

\begin{tabular}{l|cc} 
Organelle/Cellular component & Refractive Index & Reference \\
\hline Cytosol & $1.36-1.39$ & {$[69]$} \\
Nucleus & $1.355-1.365$ & {$[69]$} \\
Mitochondrion & $1.40-1.42$ & {$[70]$} \\
Lysosome & 1.60 & {$[71]$}
\end{tabular}

It has been shown using optical imaging methods that optical scatter changes occurring at the onset of apoptosis are spatially associated with mitochondria [72]. This is likely due to the roles that mitochondria play during apoptosis and the physical changes they undergo. Mitochondria break down into smaller fragments in a process known as mitochondrial fission, and though this is a normal process that occurs in viable cells, it is believed to be enhanced in cells experiencing apoptosis [64]. These smaller mitochondria likely experience enhanced diffusion throughout the cell, since an object's rate of diffusion is inversely proportional to its size. Though cellular nuclei also experience fragmentation during apoptosis, mitochondria likely play the most significant role in DLS measurements of apoptotic cells.

The main objectives of this thesis are to apply DLS-OCT techniques to detect cell death in an adherent cancer cell line in vitro and to develop a fitting model for the ACFs of cancer cell pellets. Breast adenocarcinoma cells were chosen for in vitro experiments because 
they are adherent and also because breast cancer is one of the most common forms of cancer in Canada, with over 25\% of Canadian women expected to develop it in their lives [4].

The desire to use DLS-OCT techniques to detect cell death in adherent cancer cells stems from the fact that such cells could in the future be adapted to in vivo studies, as they can be grown into tumors on small animals. For DLS-OCT to be used to monitor cancer treatment clinically or pre-clinically, it must be adapted from in vitro cell pellets to in vivo tumors to demonstrate its efficacy in real living organisms. This study, therefore, provides the next step towards that goal by testing the feasibility of DLS-OCT to detect cell death in adherent cells in vitro.

This study also aims to develop a model to fit to the ACFs generated by DLS-OCT of cell pellets using the CONTIN algorithm. Though previous work demonstrated that DLSOCT could detect cell death merely by observing how rapidly the ACFs decayed, I aspire develop a model that directly relates the ACFs from DLS-OCT measurements to biophysical phenomena within the cell pellets. Since cells contain many different organelles of different sizes, we predict the ACFs from cell pellets to be comprised of a superposition of many different exponential decays with different decorrelation times, as well as a DC signal component due to static scatterers and a delta function component corresponding to particles moving in and out of a resolution volume (see Figure 1-10).

The methods developed in this thesis could be used in principle for clinical monitoring of cell death during cancer treatments, either for superficial tumors for which tumor depths are accessible by current OCT technology, or by designing the appropriate probes that can be inserted interstitially to image the tissue pathology. The main application envisioned is for preclinical studies to assess the effectiveness of various drugs or treatments on cancerous 
masses in small animals. Since OCT has limited depth penetration due to the high optical absorption of biological tissues, clinical applications of DLS-OCT would likely be limited to superficial tumors that are located near or on the surface of the body, such as skin or mouth tumors. However, the methods will likely find use as a preclinical tool for monitoring treatment effectiveness in small animal models. Of particular interest in studies that use OCT to image tumors in small animals is the dorsal skinfold window chamber model, in which the dorsal skin of a mouse or other small animal is stretched between two plates, some of the superficial layers of the skin and muscle are removed, and the exposed region is covered with glass [73]. Tumors originating from many different organs or tissues can be implanted into the dorsal region of a mouse, and the window chamber model allows for easy imaging with OCT.

The main hypothesis of this thesis is that the DLS-OCT techniques used to detect cell death in AML cells can be applied to adherent breast cancer cells to detect responses to chemotherapy treatment and nutrient deprivation.

The specific aims of this thesis are to:

1. Develop a fitting model for the ACFs produced by DLS-OCT of cancer cell pellets using the CONTIN algorithm.

2. Use DLS-OCT to detect cell death in breast adenocarcinoma cells that have undergone various treatments:
a. No treatment/control
b. Nutrient deprivation for 24 or 48 hours
c. Treatment with chemotherapy for 24 or 48 hours 


\section{Chapter 2: Methods}

\subsection{Bead Validation Experiments}

Prior to performing in vitro experiments, DLS-OCT methods were validated by measuring the Brownian motion in monodisperse and polydisperse microsphere solutions.

\subsubsection{Brownian Motion in Monodisperse Sphere Solutions}

Polystyrene microspheres (Polysciences, Warrington, PA) with mean diameters of $0.202 \mu \mathrm{m}, 0.465 \mu \mathrm{m}$, and $0.989 \mu \mathrm{m}$ (all with a coefficient of variation of $3 \%$ ) were made into solutions with volume fractions of $2.56 \%, 1.25 \%$, and $0.53 \%$, respectively. The solutions were left for 24 hours at room temperature prior to OCT imaging to ensure uniform temperature.

OCT imaging was performed using a MEMS-VCSEL swept source OCT system from Thorlabs Inc. (see Figure 2-1) with a central wavelength of $1310 \mathrm{~nm}$, a bandwidth of $100 \mathrm{~nm}$, and an acquisition rate of 100,000 A-scans per second. The lateral and axial resolutions were approximately $25 \mu \mathrm{m}$ and $12 \mu \mathrm{m}$, respectively. Each time a solution was imaged, 100 consecutive B-mode frames were acquired at a framerate of $333 \mathrm{~Hz}$. Pixels in the image were approximately $10 \times 10 \mu \mathrm{m}$ in size. 


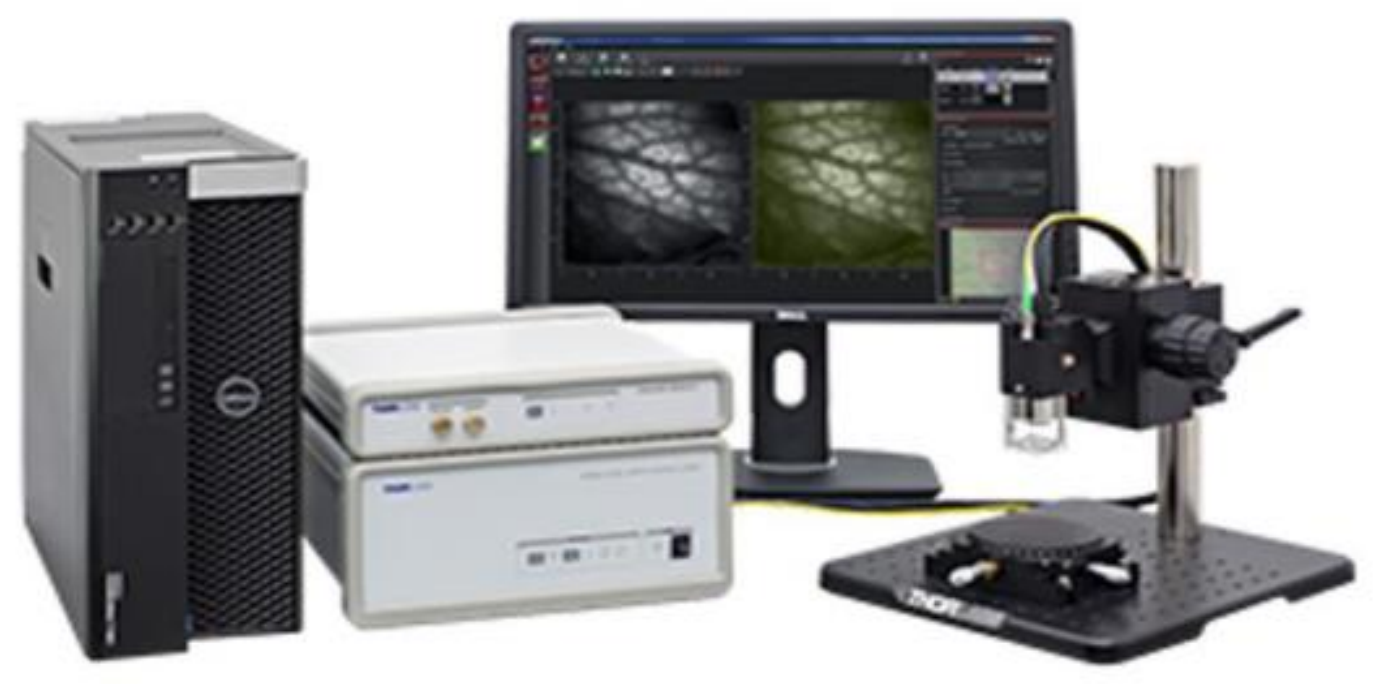

Figure 2-1. High speed MEMS-VCSEL Swept Source OCT system from Thorlabs, Inc. (Adapted from Zam et al [74]).

For each set of 100 consecutive OCT B-mode images, a region of interest (ROI) was selected for analysis. The size of the ROI was 100 pixels in the lateral direction and 3 pixels in the axial direction, corresponding to distances of about $1 \mathrm{~mm}$ and $30 \mu \mathrm{m}$, respectively. The top of the ROI was $30 \mu \mathrm{m}$ below the top surface of the sample to avoid any specular reflections.

For each of the 300 pixels in the ROI, the pixel intensity across all acquired frames was plotted and stored as a time-intensity signal. An ACF was generated from each signal by taking the inverse Fourier transform of the signal power spectrum. The mean ACF was then computed from the 300 individual ACFs to represent the average autocorrelation of the entire ROI. The mean ACF was normalized to have a maximum value of 1 for analysis. 
The ACFs were fitted to exponential decay functions to compute the decorrelation time, defined in this experiment as the time for an exponential to decay to $1 / e$ of its maximum value.

\subsubsection{Brownian Motion in Polydisperse Sphere Solutions}

Polystyrene microspheres (Polysciences, Warrington, PA) with mean diameters of $0.51 \mu \mathrm{m}$, and $1.93 \mu \mathrm{m}$ (each with a coefficient of variation of $3 \%$ ) were made into solutions with volume fractions of $0.53 \%$ each. A third solution was prepared with both bead sizes mixed into the same solution at volume fractions of $0.26 \%$ each. The solutions were left for 24 hours at room temperature prior to OCT imaging to ensure a steady state temperature.

The methods used to image the beads and compute ACFs are described in Section 2.1.1. After the ACF acquisition, the CONTIN algorithm was used to compute the decorrelation time distributions from each of the three samples. The range of decorrelation times used as input for the CONTIN algorithm consisted of 200 points evenly spaced in logspace between 0.1 and $100 \mathrm{~ms}$. The regularization parameter was first set to 0 to maximize the peak-separating resolution of the algorithm. Since the sizes of the beads were known $a$ priori, true peaks could easily be distinguished from phantom peaks. The ACFs were fed as input into the CONTIN algorithm a second time, using a regularizer of 0.1 to remove phantom peaks. 


\subsection{Detecting Cell Death using DLS-OCT}

Each set of in vitro experiments was completed over a course of four days, excluding the time it took to grow the cells to the initial starting population size. In total, there were three experimental repetitions of the process described in 2.2.1.

\subsubsection{Biological Sample Preparation and Imaging Timeline}

Day 1

Cell samples were prepared from breast ductal carcinoma cells (MCF-7). Approximately $2 \times 10^{7}$ cells were grown at $37^{\circ} \mathrm{C}$ in a $75 \mathrm{~cm}^{2}$ cell culture flask containing $10 \mathrm{~mL}$ of Dulbecco's Modified Eagle Medium (DMEM) with $4.5 \mathrm{~g} / \mathrm{L}$ of glucose and supplemented with $10 \%$ fetal bovine serum, $1 \%$ penicillin and streptomycin, and $1 \%$ insulin. 24 hours prior to the start of any treatments, the cells were split into three samples of approximately $7 \times 10^{6}$ cells in three separate $75 \mathrm{~cm}^{2}$ cell culture flasks, each containing the same volume and concentration of media and supplements as the initial flask, but without insulin ${ }^{1}$.

Day 2

After 24 hours, the cells had completely adhered to the bottom surface of the flasks and to each other, forming monolayers in each of the three flasks. Two of the samples were

\footnotetext{
${ }^{1}$ Miglietta et al [67] demonstrated that insulin inhibits MCF-7 cell sensitivity to paclitaxel. They observed that approximately $50 \%$ of cells in a sample were viable after being treated wit $10 \mathrm{nM}$ paclitaxel for 24 hours, but as many as $75 \%$ of cells were viable after being treated with both $10 \mathrm{nM}$ paclitaxel and $20 \mathrm{ng} / \mathrm{mL}$ of insulin simultaneously. In our experiment, we removed insulin for 24 hours before treating cells with paclitaxel to mitigate the paclitaxel resistance-enhancing effects of insulin.
} 
treated with $20 \mathrm{ng} / \mathrm{mL}$ of paclitaxel, a chemotherapeutic drug that is commonly used to treat breast cancer and is classified as an anti-microtubule agent. Paclitaxel activates adenosine monophosphate-activated protein kinase (AMPK), which plays a role in suppressing breast tumor growth via the regulation of downstream pathways [76]. Cells treated with paclitaxel often have defects in mitotic spindle assembly and cell division, which triggers mitotic arrest and leads to apoptosis. Saunders et al [77] found that at a concentration of $20 \mathrm{ng} / \mathrm{mL}$, paclitaxel inhibited MCF-7 cell growth in vitro by approximately $75 \%$, and that after such a treatment approximately $43 \%$ of cells were apoptotic, but concentrations higher than 20 $\mathrm{ng} / \mathrm{mL}$ did not further increase apoptosis (see Figure 2-2). After adding the treatment, cell samples were incubated at $37^{\circ} \mathrm{C}$.

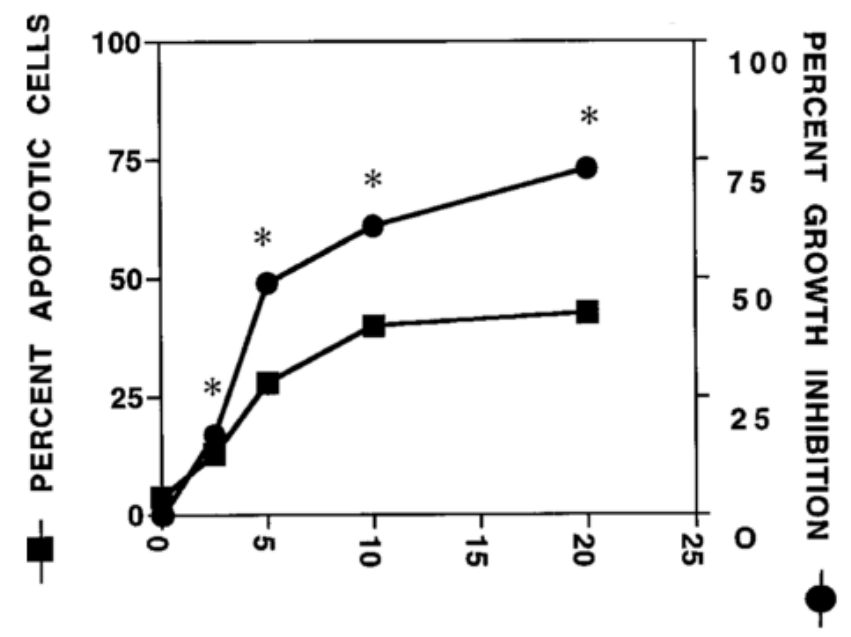

PACLITAXEL $(\mathrm{ng} / \mathrm{ml})$

Figure 2-2. The effects of various concentrations of paclitaxel on MCF-7 breast cancer cells. At concentrations higher than $20 \mathrm{ng} / \mathrm{mL}$, there is no significant increase in the percent of apoptotic cells. (Adapted from [77]). 
The untreated cells were formed into a cell pellet and imaged with OCT. To form a cell pellet, the sample was first washed with 1x phosphate buffered saline (PBS) and treated with $0.25 \%$ trypsin and ethylenediaminetetraacetic acid (EDTA) for 5 minutes. Trypsin is a digestive enzyme that is formed in the small intestine and cleaves proteins. In cell culture, it is used to suspend cells that are adherent to the surface of a flask by cleaving the proteins that attach the cells to the dish. EDTA is a chemical that binds to cadherins, which are proteins that link cells to one another. It is used to separate cells that adhere to each other. Once the cells were suspended, DMEM was added to them to neutralize the Trypsin, and then they were centrifuged at $200 \mathrm{G}$ for 3 minutes to separate them from the media. They were then placed into a small pellet container and spun at $500 \mathrm{G}$ for 10 minutes to form a tightly-packed cylindrical cell pellet with a height of approximately $0.6-0.8 \mathrm{~mm}$ and a diameter of $8 \mathrm{~mm}$.

After imaging the untreated cells, a thin layer of PBS was added on top of the cells to prevent them from drying out, and the cells were placed in the incubator at $37^{\circ} \mathrm{C}$.

Day 3

The untreated cells, already in pellet form, were imaged with OCT a second time after 24 hours of nutrient deprivation. Prior to imaging, the PBS on top of the cell pellet was carefully removed with a micropipette. After imaging, the cell pellet was covered with a thin layer of PBS again and placed in the incubator at $37^{\circ} \mathrm{C}$.

One of the paclitaxel-treated cell samples was made into a pellet and imaged with OCT after 24 hours of treatment. The protocol for pellet formation was the same as it was for the untreated cells. After imaging, the paclitaxel treated cell pellet was discarded. 
Day 4

The untreated cells, still in pellet form, were imaged with OCT after 48 hours of nutrient deprivation. Prior to imaging, the PBS on top of the cells were once again removed with a micropipette. After imaging, the cell pellet was discarded.

The remaining paclitaxel-treated cell sample was made into a pellet and imaged with OCT after 48 hours of treatment. After imaging, the pellet was discarded.

\subsubsection{OCT System and Acquisition Methods}

The same OCT system was used in in vitro experiments as in the validation experiments. Each time a cell pellet was imaged, 500 consecutive B-mode frames were acquired at a framerate of $333 \mathrm{~Hz}$. Furthermore, each pellet was imaged at 10 different spatial locations to account for possible heterogeneity in the pellet. Pixels in the image were approximately $10 \times 10 \mu \mathrm{m}$ in size.

\subsubsection{Data Processing and Analysis}

For each set of 500 consecutive OCT B-mode images, a region of interest (ROI) was selected for analysis (see Error! Reference source not found.). The size of the ROI was 100 pixels in the lateral direction and 3 pixels in the axial direction, corresponding to distances of about $1 \mathrm{~mm}$ and $30 \mu \mathrm{m}$, respectively. The top of the ROI was $30 \mu \mathrm{m}$ below the top surface of the sample to avoid any specular reflections from the surface.

For each of the 300 pixels in the ROI, the pixel intensity across all acquired frames was plotted and stored as a time-intensity signal (see Error! Reference source not found.b). 
An ACF was generated from each signal by taking the inverse Fourier transform of the signal power spectrum. The mean ACF was then computed from the 300 individual ACFs to represent the average autocorrelation of the entire ROI. The mean ACF was normalized to have a maximum value of 1 for simplicity and normalized to decay to a minimum value of 0 to remove the contribution from static scatterers, which are known to contribute a constant value to the ACF.

The mean ACFs decayed to $50 \%$ or less of their maximum value within one $3 \mathrm{~ms}$ time step (see Section Error! Reference source not found.), so the decorrelation time was not defined as the time for the ACF to decay to half its maximum value, as was done by Farhat et al [43]. Instead, decorrelation times were calculated for each set of data by fitting the mean ACFs to exponential curves (see Equation 6), as was previously done by our lab [74]. The decorrelation times calculated in this study therefore represent the times for fitted exponentials to decay to $1 / e$ of their respective maximum values. 

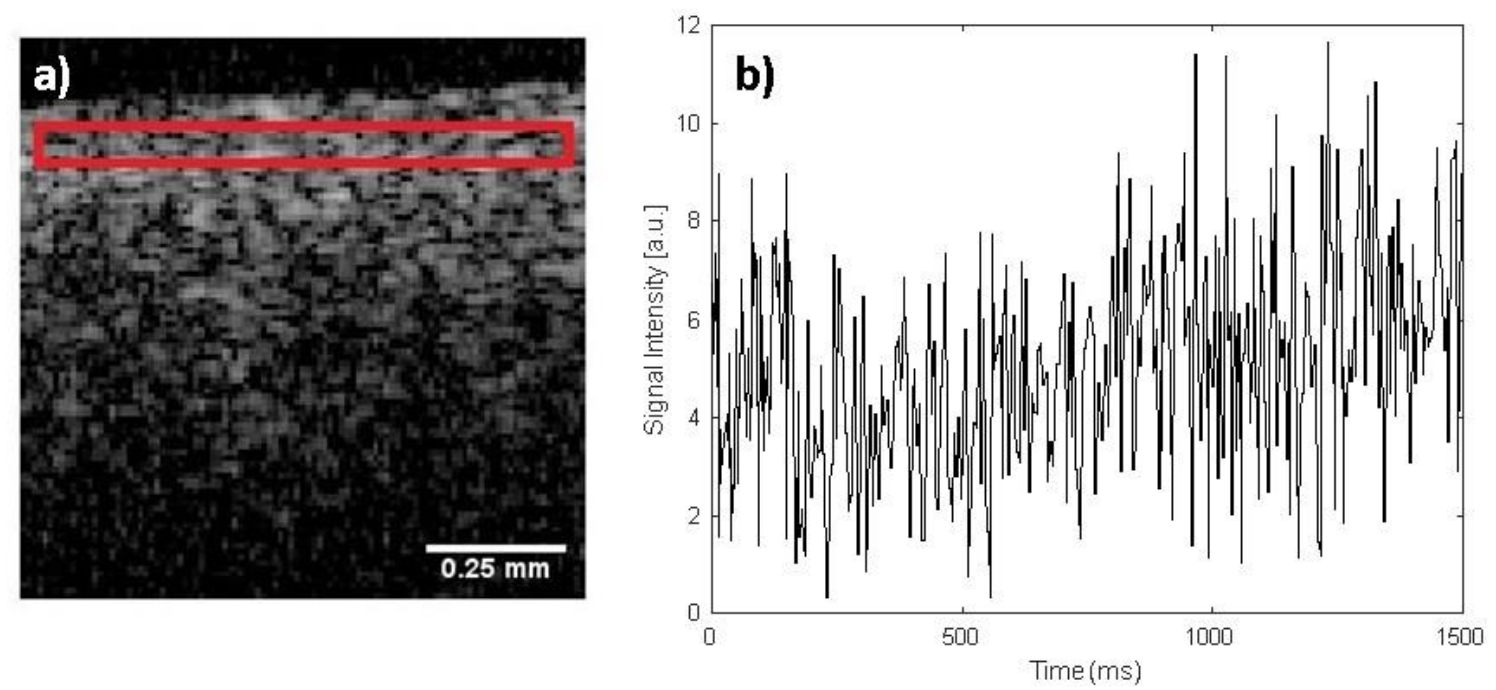

Figure 2-3. (a): OCT B-mode image of an untreated MCF-7 cancer cell pellet. The image depicts a crosssectional view of the cell pellet. The ROI is outlined by the red rectangle. (b): Signal intensity as a function of time for a single pixel in the ROI from (a). 


\subsection{Using CONTIN to Determine Decorrelation Time Distributions}

CONTIN was used to generate decorrelation time distributions for each of the final mean ACFs acquired in the experiments discussed in section 2.2. In total, there were 150 ACFs: 30 from untreated cells, 30 each from cells deprived of nutrients for 24 and 48 hours, and 30 each from cells treated with $20 \mathrm{ng} / \mathrm{mL}$ paclitaxel for 24 and 48 hours.

Cells contain many different scatterers of different sizes and movement speeds, such as mitochondria, lysosomes, cellular nuclei, and cytoskeletal components. As such, cells have very complex scattering profiles, and the number of peaks that would appear in the decorrelation time distributions was not known a priori and was not entered as a parameter into CONTIN.

To determine the regularization parameter used in the CONTIN simulations, the following criteria was used:

1. An initial CONTIN run was performed on five randomly selected ACFs using a regularization parameter of 0 . A regularizer of 0 gives the best resolved distribution at the expense of potential phantom peaks.

2. If any of the peaks in the five output decorrelation time distributions were not fully resolved ${ }^{2}$ (see Figure 2-4), the regularization parameter was increased by 0.01 and CONTIN was performed again.

3. Step 2 was repeated until only full resolvable peaks remained.

\footnotetext{
2 In this study, we defined the term fully resolvable as follows: two consecutive peaks are fully resolvable if they do not overlap. In other words, there exists a decorrelation time between the two peaks with an intensity contribution of $0 \%$.
} 
By having distributions only containing fully resolved peaks, it is easier to compare the distributions resulting from different cell populations, such as untreated cells and nutrientdeprived cells. When peaks are not fully resolvable and overlap, it becomes difficult to calculate the center of each peak and the area under the curve (AUC) of each peak, both of which are metrics that are used to compare the peaks from different distributions.

The regularizer chosen for the CONTIN fitting was 0.1 and all 150 decorrelation time distributions were computed using this value to maintain consistency. The range of possible decorrelation times was set to be 0.1 to $1000 \mathrm{~ms}$.
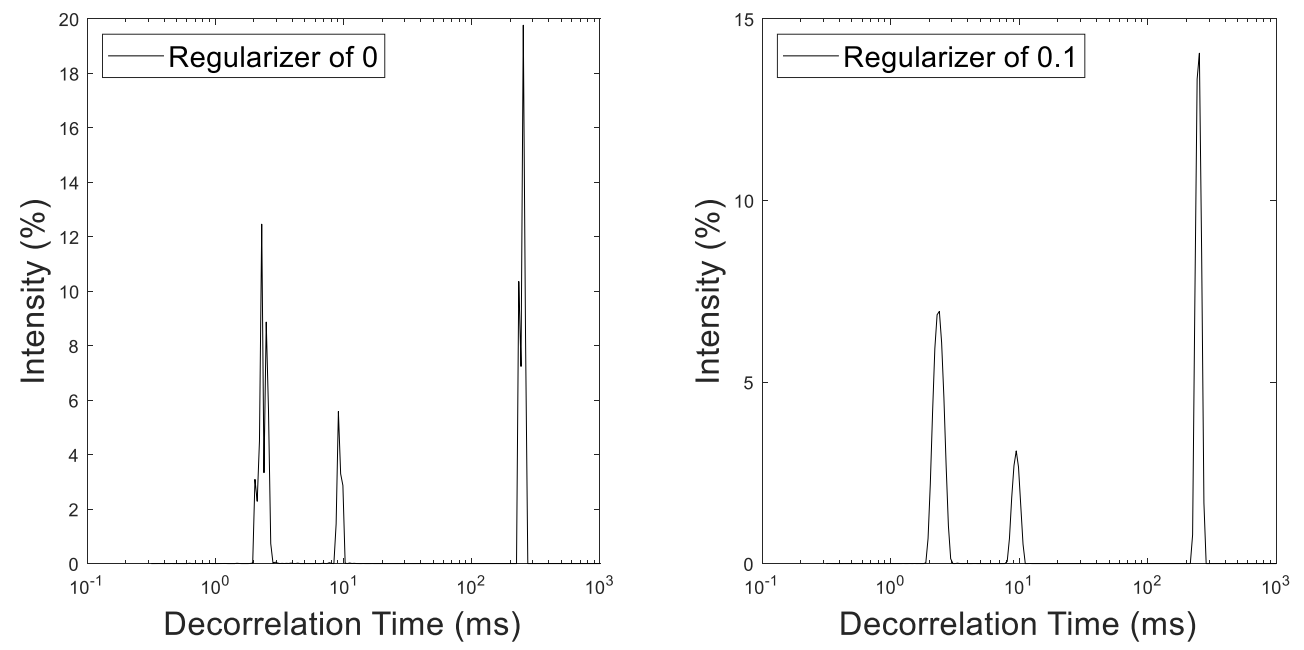

Figure 2-4. CONTIN distributions of an ACF from untreated breast cancer cells. When the regularizer was set to 0 (left), there were overlapping peaks, but when the regularizer was set to 0.1 (right), all peaks were clearly defined and separated. 


\section{Chapter 3: Results}

\subsection{Bead Validation Experiments}

OCT images of the three different monodisperse microbead solutions are shown in Figure 3-1. The ACFs and decorrelation times from the three samples are shown in Figure 3-2. Each sample was imaged three times, at three different spatial locations. The ACFs decay more rapidly the smaller the particles are. The $0.465 \mu \mathrm{m}$ and $0.989 \mu \mathrm{m}$ bead solutions produced decorrelation times that matched the predicted values of the Einstein-Stokes equation, but the $0.202 \mu \mathrm{m}$ beads produced a higher value than expected. This deviation from the expected value is likely an aliasing artifact from under-sampling. The frame rate of the OCT device used in this experiment was $333 \mathrm{~Hz}$, which corresponds to an acquisition time of $3 \mathrm{~ms}$ per frame. The $0.202 \mu \mathrm{m}$ bead solution has a predicted decorrelation time less of than $3 \mathrm{~ms}$, which means it is possible that an OCT system with a higher frame rate could more accurately resolve the ACF for the bead solution.
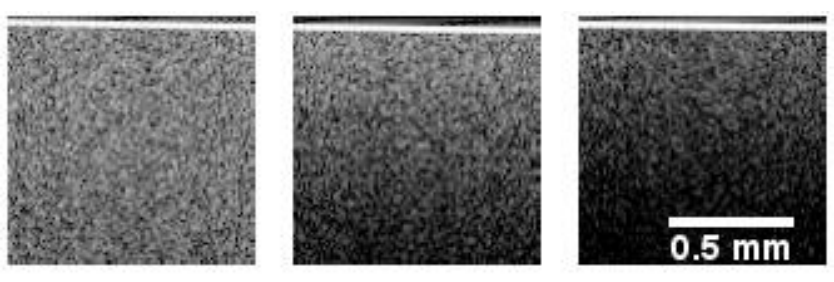

Figure 3-1. OCT cross-sectional images of monodisperse bead solutions. From left to right: $0.202 \mu$ m diameter beads, $0.465 \mu \mathrm{m}$ diameter beads, and $0.989 \mu \mathrm{m}$ diameter beads. Image intensity is normalized on a scale from 0 (black) to 255 (white). 

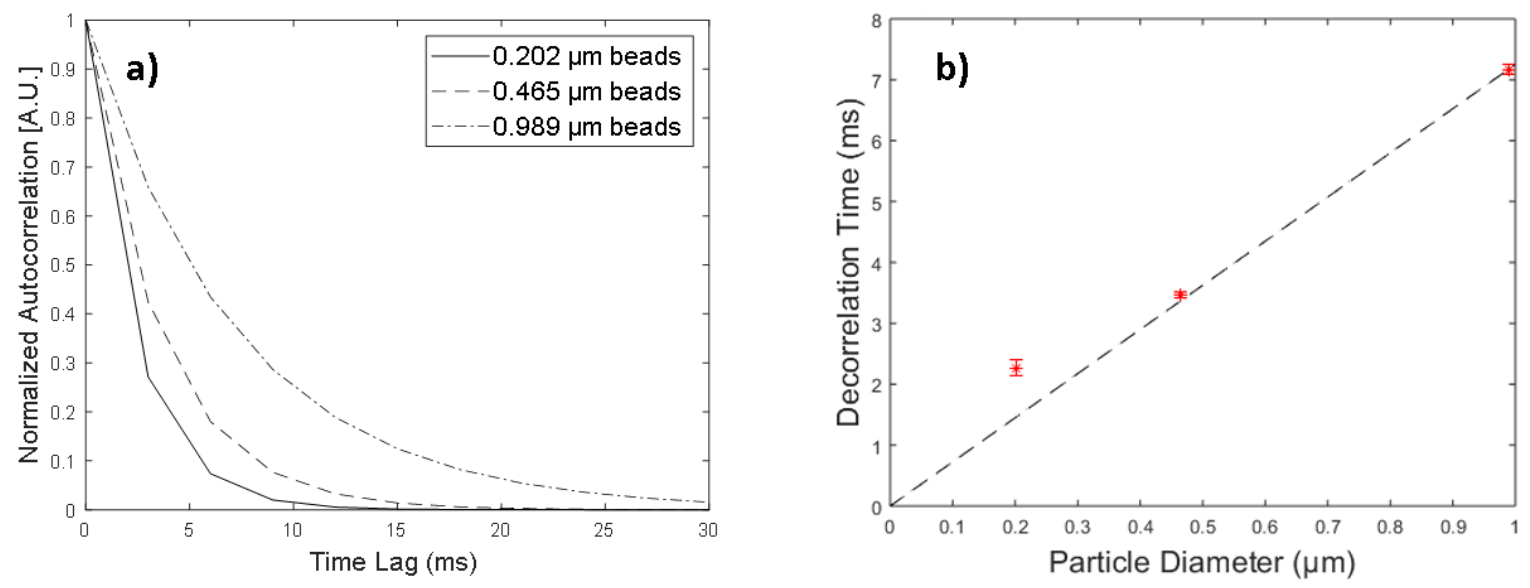

Figure 3-2. a): Average ACFs obtained from monodisperse microsphere solutions. b): Decorrelation time vs. particle diameter for DLS-OCT analysis of monodisperse microsphere solutions. Error bars represent the standard deviation from measurements at three spatial locations. The dotted line depicts the predicted decorrelation times as per the Einstein-Stokes equation.

An OCT image of the polydisperse bead solution is shown in Figure 3-3. Each sample was imaged three times. The mean ACFs from the polydisperse solution and from two monodisperse solutions containing $0.51 \mu \mathrm{m}$ beads and $0.93 \mu \mathrm{m}$ beads, respectively, are displayed in Figure 3-4. As expected, the ACF from the polydisperse solution decays faster than the ACF from the monodisperse $1.93 \mu \mathrm{m}$ bead solution, but slower than the ACF from the monodisperse $0.51 \mu \mathrm{m}$ bead solution. 


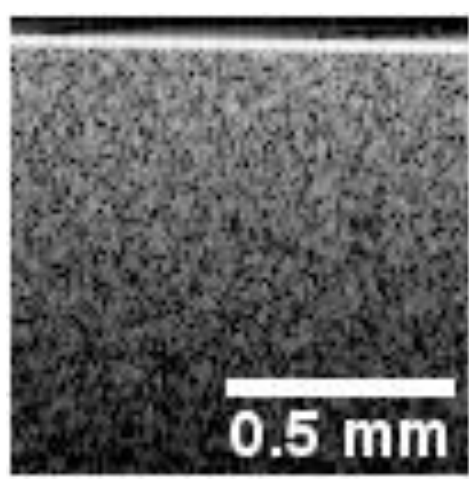

Figure 3-3. OCT cross-sectional image of the polydisperse microsphere solution containing $0.51 \mu \mathrm{m}$ and 1.93 $\mu \mathrm{m}$ diameter beads mixed at a 1:1 volume ratio.

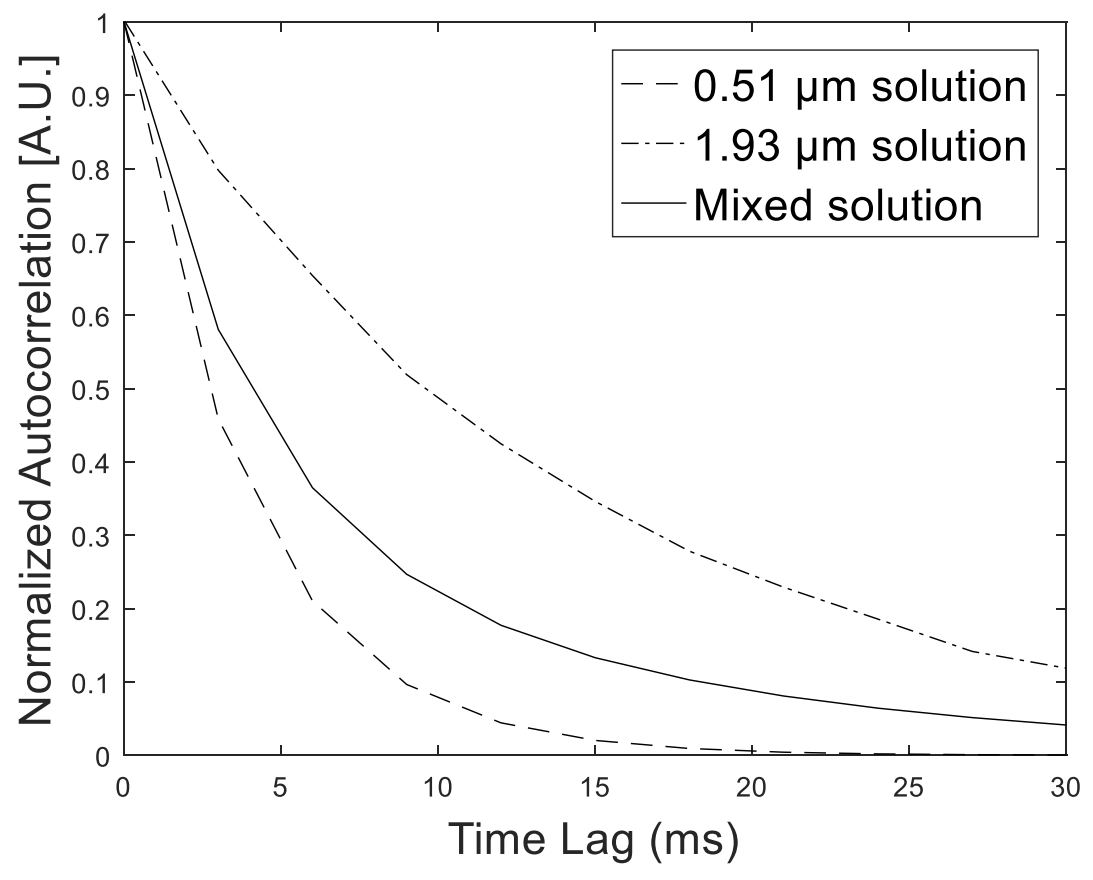

Figure 3-4. Average ACFs obtained from monodisperse solutions of $0.53 \mu \mathrm{m}$ and $1.93 \mu \mathrm{m}$ diameter beads, and polydisperse solution containing both bead sizes. 
The results from performing the CONTIN algorithm on the three ACFs are displayed in Figure 3-5 and Figure 3-6. The decorrelation time distribution functions computed using CONTIN for the monodisperse solutions each have a single cluster of peaks, located between 3-5 ms for the $0.51 \mu \mathrm{m}$ beads and between $10-20 \mathrm{~ms}$ for the $1.93 \mu \mathrm{m}$ beads. These match the expected values, computed using the Einstein-Stokes equation. Using the CONTIN algorithm we could accurately determine that the ACFs came from monodisperse samples with no $a$ priori information about the number of peaks that should appear in the distribution, and we were able to accurately determine the decorrelation times.

CONTIN produced two peak clusters for the ACF data acquired from the polydisperse solution, again located between 3-5 ms and 10-20 ms. The CONTIN algorithm was able to determine the number of different particle sizes in the solution and the approximate decorrelation times for each of those groups. These results indicate that CONTIN can be used to compute the decorrelation time distribution from an ACF acquired via DLS-OCT with good accuracy, but with limited precision. 

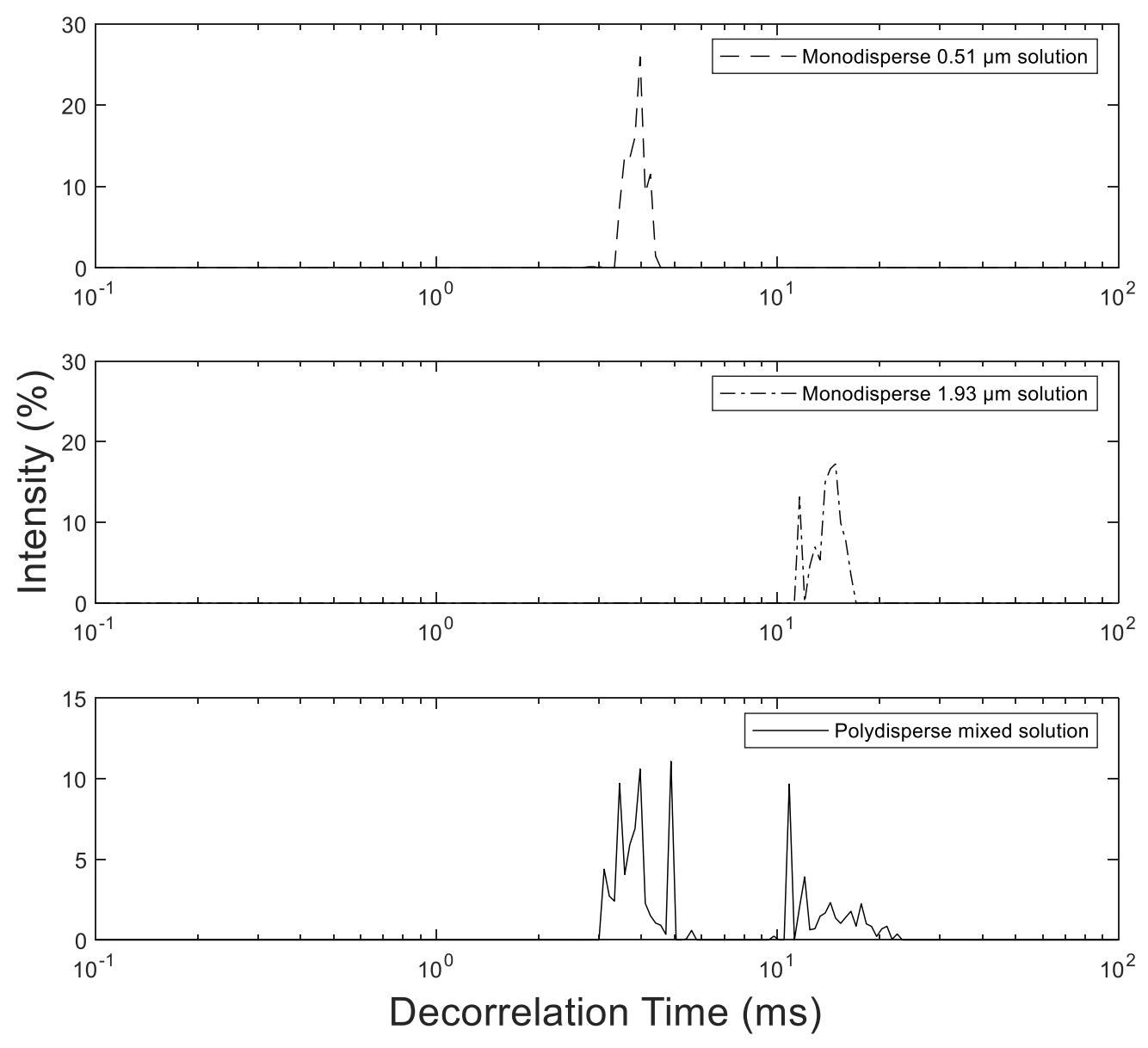

Figure 3-5. Decorrelation time distribution functions for polystyrene bead solutions. The distributions were computed with CONTIN, using a regularizer of 0. 

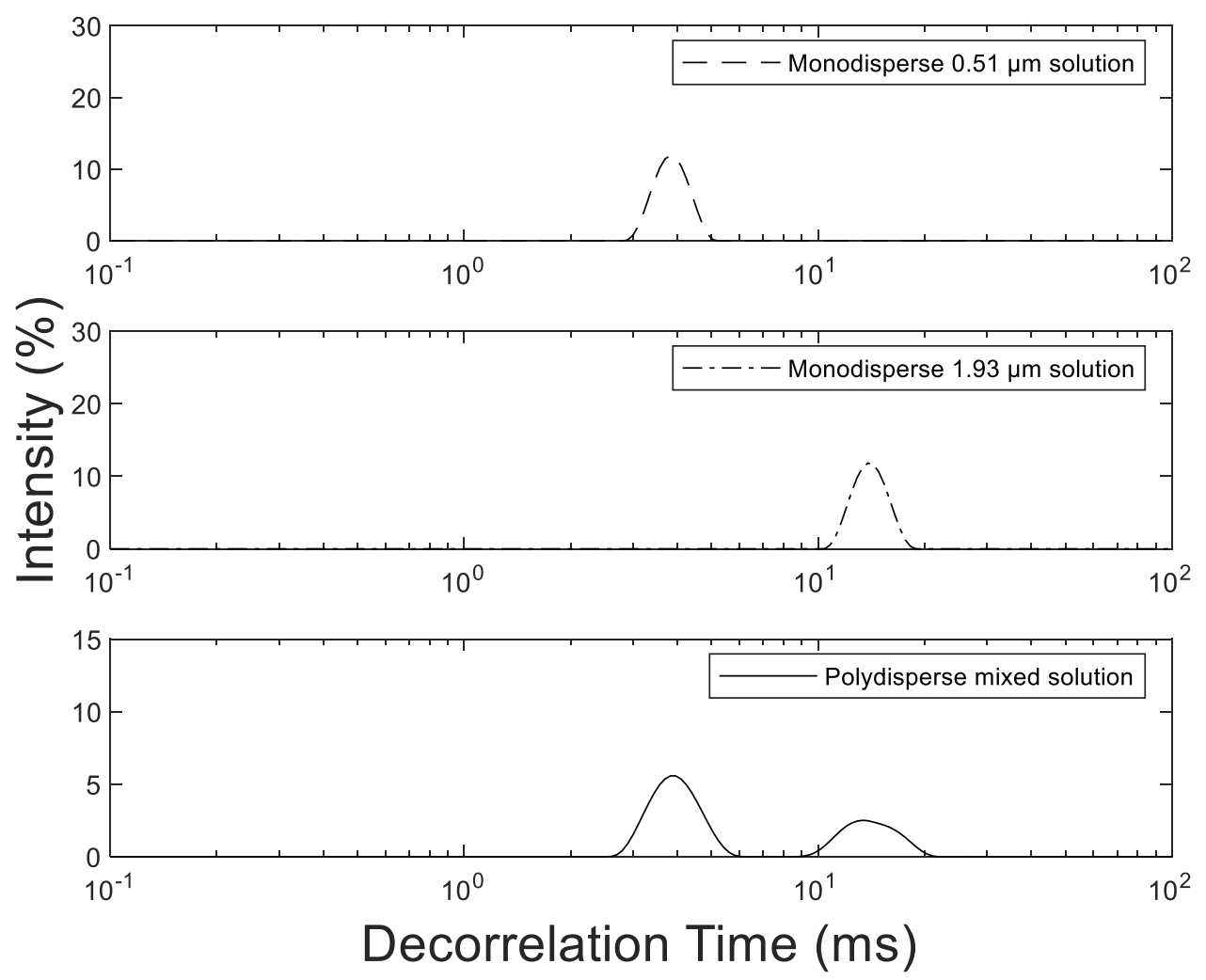

Figure 3-6. Decorrelation time distribution functions for polystyrene bead solutions. The distributions were computed with CONTIN, using a regularizer of 0.1. 


\subsection{Detecting Cell Death Using DLS-OCT}

The results of this study are divided into two sections: the results of treating breast cancer cells with paclitaxel, and the results of depriving breast cancer cells of nutrients for various periods of time.

\subsubsection{Paclitaxel-Treated Breast Cancer Cells}

The average ACFs of cancer cell pellets from one experimental trial are displayed in Figure 3-7. Average decorrelation time as a function of treatment duration for all three trials is plotted in Figure 3-8 and listed in Table 3-1. A large decrease in decorrelation time was observed in the cells after 24 hours of paclitaxel exposure, and a further drop was observed after 48 hours of paclitaxel exposure. T-tests revealed the drop from untreated to 24 hour treated cells and from untreated to 48 hour treated cells to be statistically significant across all trials $(\mathrm{p} \leq 0.002)$. However, the decrease in decorrelation time from 24 hours of treatment to 48 hours of treatment was not statistically significant in all trials $(\mathrm{p}>0.05)$. Decorrelation time frequency distributions for each treatment duration are shown in Figure 3-9.

We hypothesize that the change in decorrelation time observed in the paclitaxeltreated breast cancer cells is a result of increased intracellular motion in the cells undergoing apoptosis. During apoptosis, a cell experiences ATP-driven processes that reorganize the cytoskeleton and cause organelles to fragment into small pieces, resulting in an overall increase in motion. After apoptosis, the cell and its organelles have been reduced to small fragments, which can experience faster diffusive motion. Though paclitaxel is known to induce apoptosis in MCF-7 breast cancer cells, it is possible that other modes of cell death, 
such as oncosis, or other processes like mitotic arrest contributed to the DLS-OCT measurements. In future work, histological confirmation or flow cytometry measurements can provide an indication of the relative frequency of modes of cell death.

The results from all three trials demonstrated very good repeatability of this experiment despite the biological variations inherent in cell samples. In each repetition of the experiment, a decrease in decorrelation time was observed after 24 hours of treatment and persisted after 48 hours of treatment.

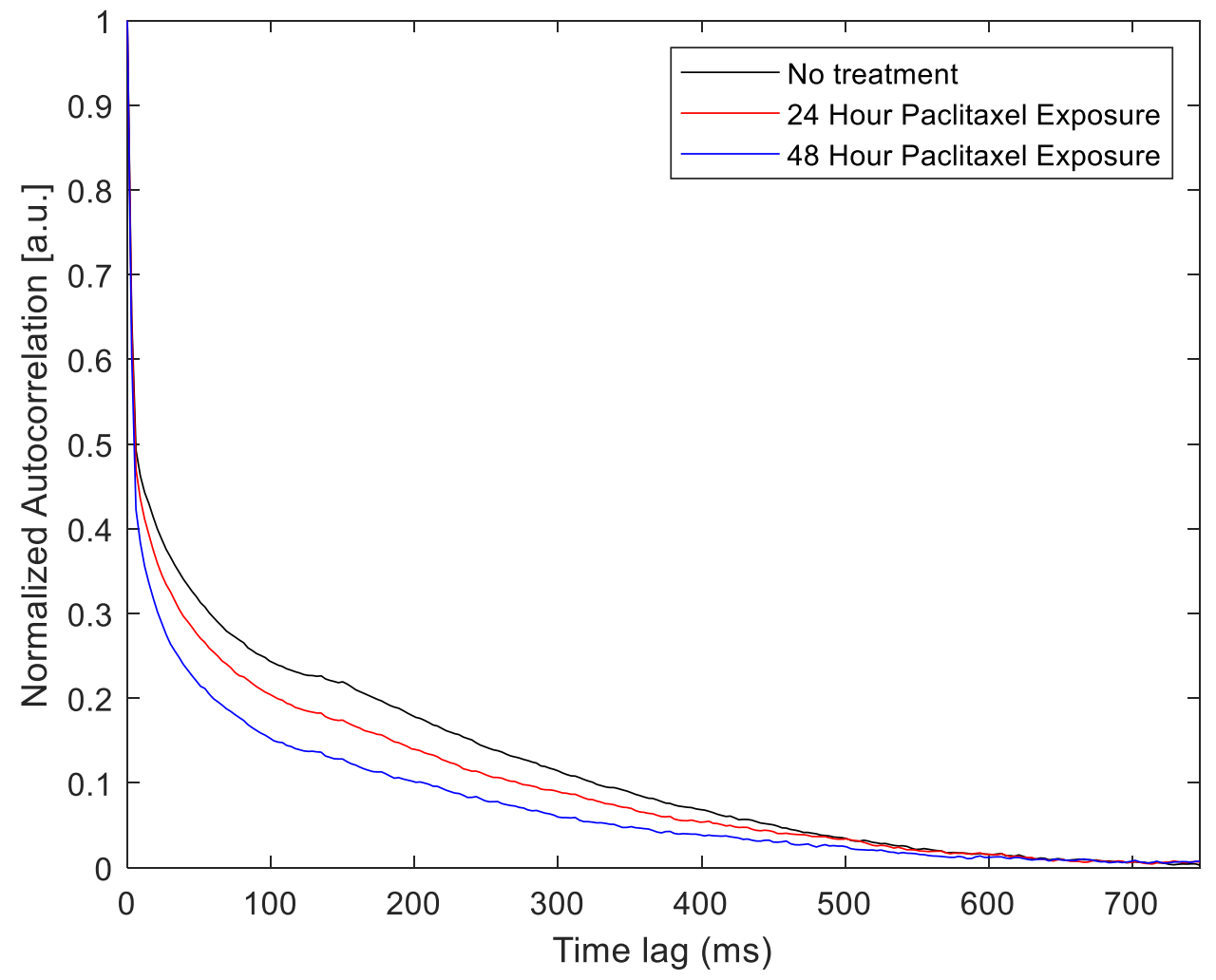


Figure 3-7. Normalized mean ACFs from cells that have been treated with paclitaxel. Each curve represents the average of 10 ACFs from different points in a sample, which in turn represents the average ACF from 300 pixels within a ROI.

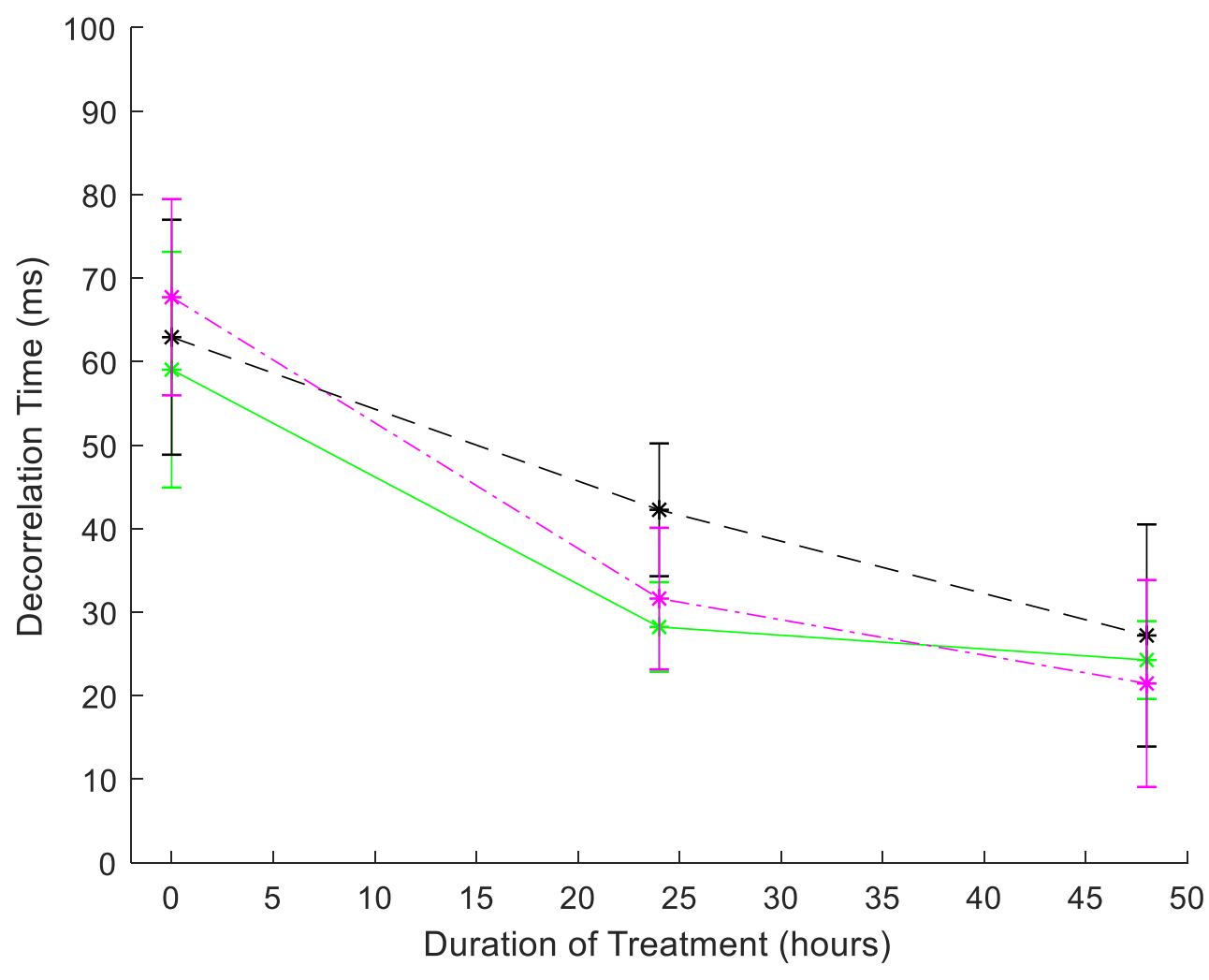

Figure 3-8. Decorrelation time as a function of paclitaxel treatment time. Each line (green, black, and magenta) represents a different trial of the same repeated experiment, all performed on separate weeks and with different cell samples. The error bars represent the standard deviation of 10 measurements. 
Table 3-1. Average decorrelation times of paclitaxel-treated cells. Each trial took place on a different week and used cells from a different passage number. In brackets are the percent changes in decorrelation time from 0 hours to either 24 or 48 hours.

\begin{tabular}{|c|c|c|c|}
\hline & Untreated & 24 hours & 48 hours \\
\hline Trial 1 & $59.03 \pm 14.11 \mathrm{~ms}$ & $\begin{array}{l}28.22 \pm 6.08 \mathrm{~ms} \\
(-52.2 \%)\end{array}$ & $\begin{array}{l}24.26 \pm 4.66 \mathrm{~ms} \\
(-58.9 \%)\end{array}$ \\
\hline Trial 2 & $62.93 \pm 14.06 \mathrm{~ms}$ & $\begin{array}{l}42.25 \pm 7.95 \mathrm{~ms} \\
(-32.9 \%)\end{array}$ & $\begin{array}{l}27.20 \pm 13.30 \mathrm{~ms} \\
(-56.8 \%)\end{array}$ \\
\hline Trial 3 & $67.71 \pm 11.75 \mathrm{~ms}$ & $\begin{array}{l}31.62 \pm 8.48 \mathrm{~ms} \\
(-53.3 \%)\end{array}$ & $\begin{array}{l}21.45 \pm 12.39 \mathrm{~ms} \\
(-68.3 \%)\end{array}$ \\
\hline
\end{tabular}
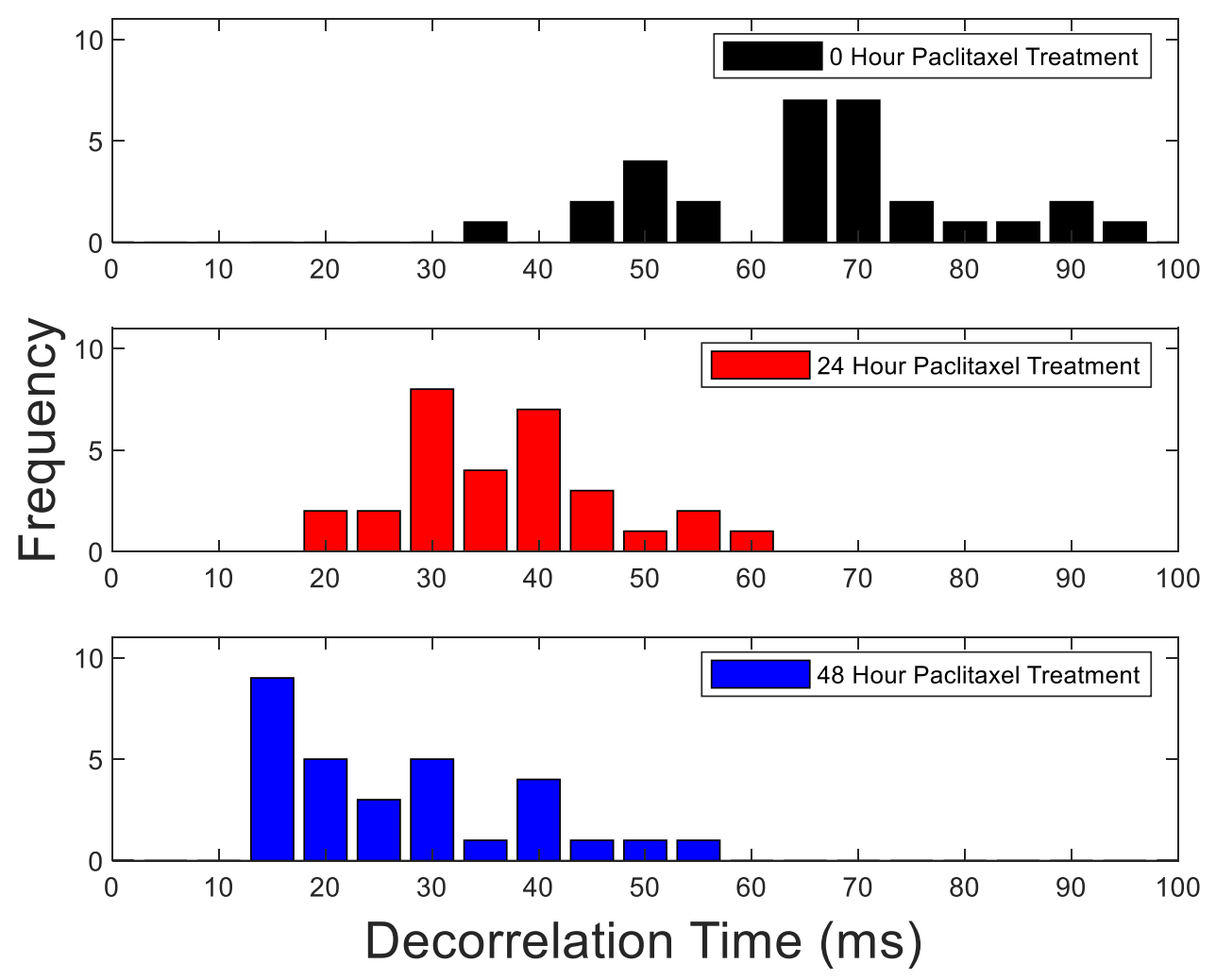
Figure 3-9. Decorrelation time frequency histograms for cells treated with paclitaxel. For each treatment duration, 30 decorrelation times were acquired, with 10 from each experimental trial. Bins are 5 ms wide.

\subsubsection{Nutrient-Deprived Breast Cancer Cells}

Average ACFs from one experimental trial of cancer cell pellet nutrient deprivation are displayed in Figure 3-10 and average decorrelation time as a function of nutrient deprivation time for all three trials is plotted in Figure 3-11 and listed in Table 3-2. As with the paclitaxel-treated cells, a large decrease in decorrelation time was observed in the nutrient-deprived cell pellets after 24 and 48 hours. The decrease in the decorrelation time from 0 hours to 24 hours and from 0 hours to 48 hours were statistically significant in all trials ( $\mathrm{p} \leq 0.0001)$, but the decrease from 24 to 48 hours was not $(\mathrm{p}>0.05)$. Decorrelation time frequency distributions for each nutrient-deprivation duration are shown in Figure 3-12.

We hypothesize that the change in decorrelation time observed in the nutrientdeprived cells is the result of the cells experiencing morphological changes due to oncosis. Unlike apoptosis, oncosis is not a programmed form of cell death and isn't accompanied by deliberate ATP-driven actions. However, oncotic cells experience swelling due to an increase in the volume of intracellular fluid. Within these expanded cells, there could be an increase in diffusive motion, since organelles and other small structures are less tightly contained. Furthermore, when the cells rupture, their contents are no longer contained within a membrane and therefore can experience even greater rates of diffusive motion.

Once again, the results of the three trials demonstrated good repeatability, with a decrease in decorrelation time being observed in all three repetitions of the experiment. 


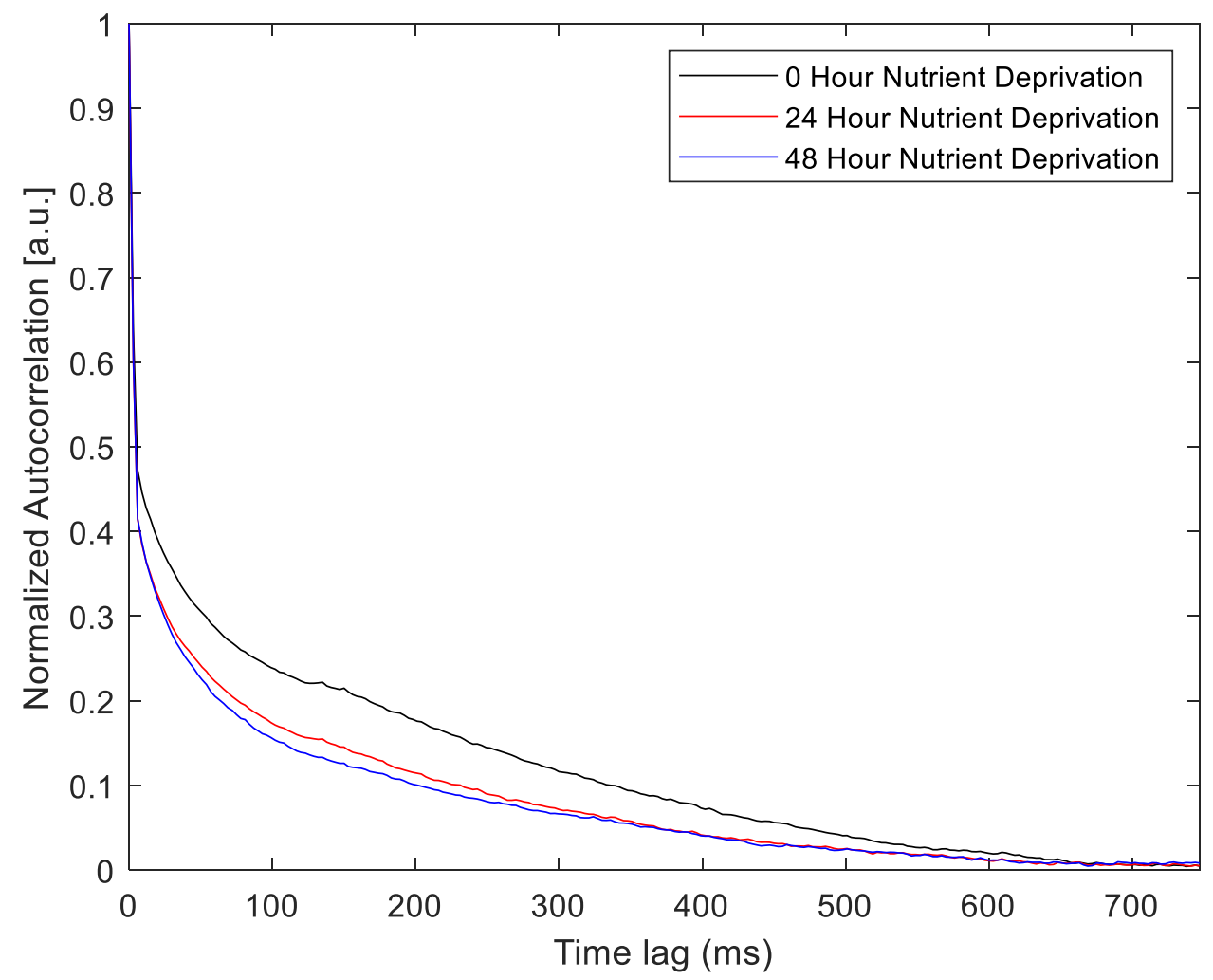

Figure 3-10. Normalized mean ACFs from cells that have been deprived of nutrients. Each curve represents the average of 10 ACFs from different points in a sample, which in turn represents the average ACF from 300 pixels within a ROI. 


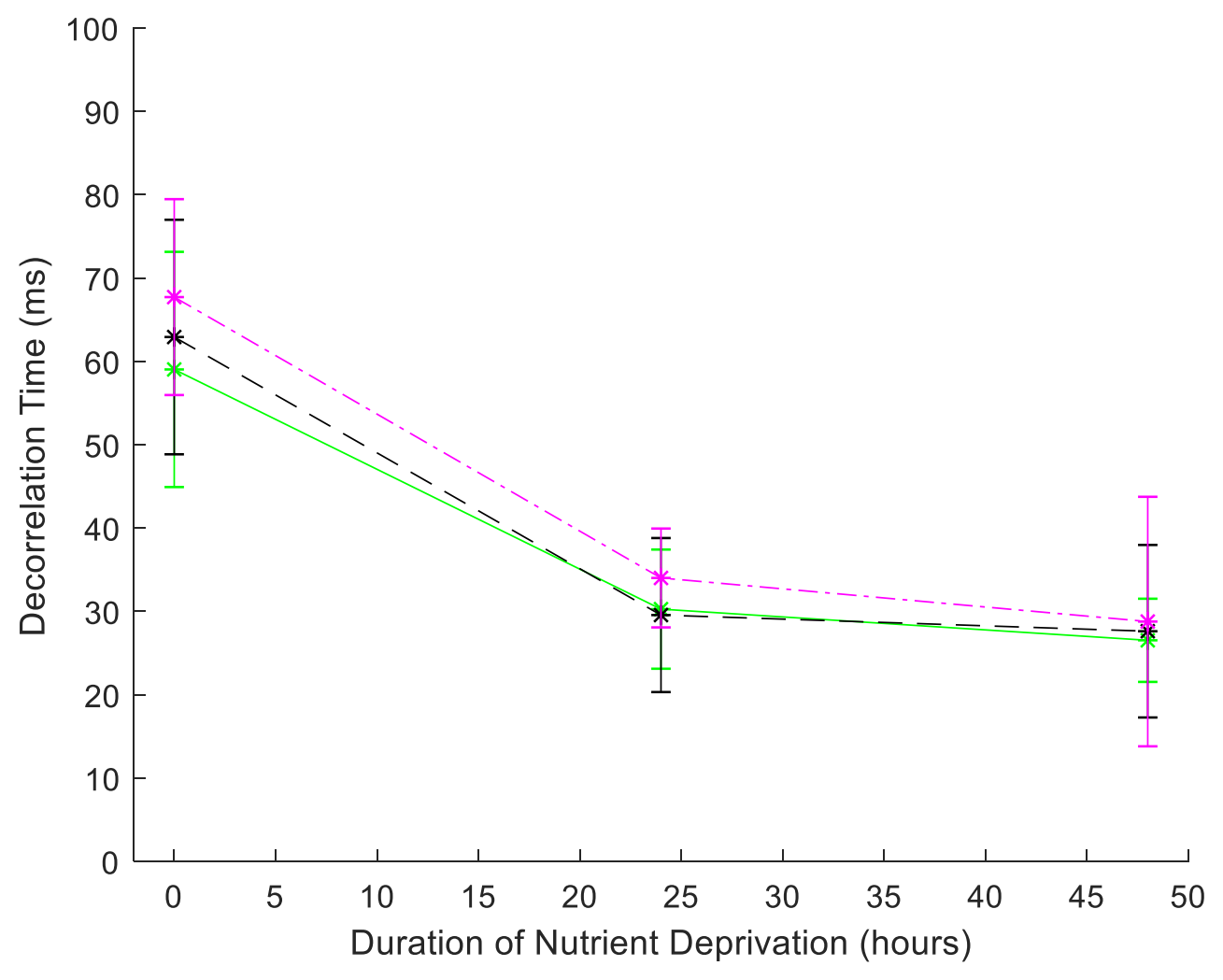

Figure 3-11. Decorrelation time as a function of nutrient deprivation time. Each line (green, black, and magenta) represents a different trial of the same repeated experiment, all performed on separate weeks and with different cell samples. The error bars represent the standard deviation of 10 measurements. 
Table 3-2. Average decorrelation times of nutrient-deprived cells. Each trial took place on a different week and used cells from a different passage number. In brackets are the percent changes in decorrelation time from 0 hours to either 24 or 48 hours.

\begin{tabular}{|l|l|l|l|}
\hline & 0 hours & 24 hours & 48 hours \\
\hline Trial 1 & $59.03 \pm 14.11 \mathrm{~ms}$ & $\begin{array}{l}30.27 \pm 7.15 \mathrm{~ms} \\
(-48.7 \%)\end{array}$ & $\begin{array}{l}26.53 \pm 4.99 \mathrm{~ms} \\
(-55.1 \%)\end{array}$ \\
\hline Trial 2 & $62.93 \pm 14.06 \mathrm{~ms}$ & $\begin{array}{l}29.56 \pm 9.24 \mathrm{~ms} \\
(-53.0 \%)\end{array}$ & $\begin{array}{l}27.62 \pm 10.35 \mathrm{~ms} \\
(-56.1 \%)\end{array}$ \\
\hline Trial 3 & $67.71 \pm 11.75 \mathrm{~ms}$ & $\begin{array}{l}34.01 \pm 5.93 \mathrm{~ms} \\
(-49.8 \%)\end{array}$ & $\begin{array}{l}28.78 \pm 14.97 \mathrm{~ms} \\
(-57.5 \%)\end{array}$ \\
\hline
\end{tabular}
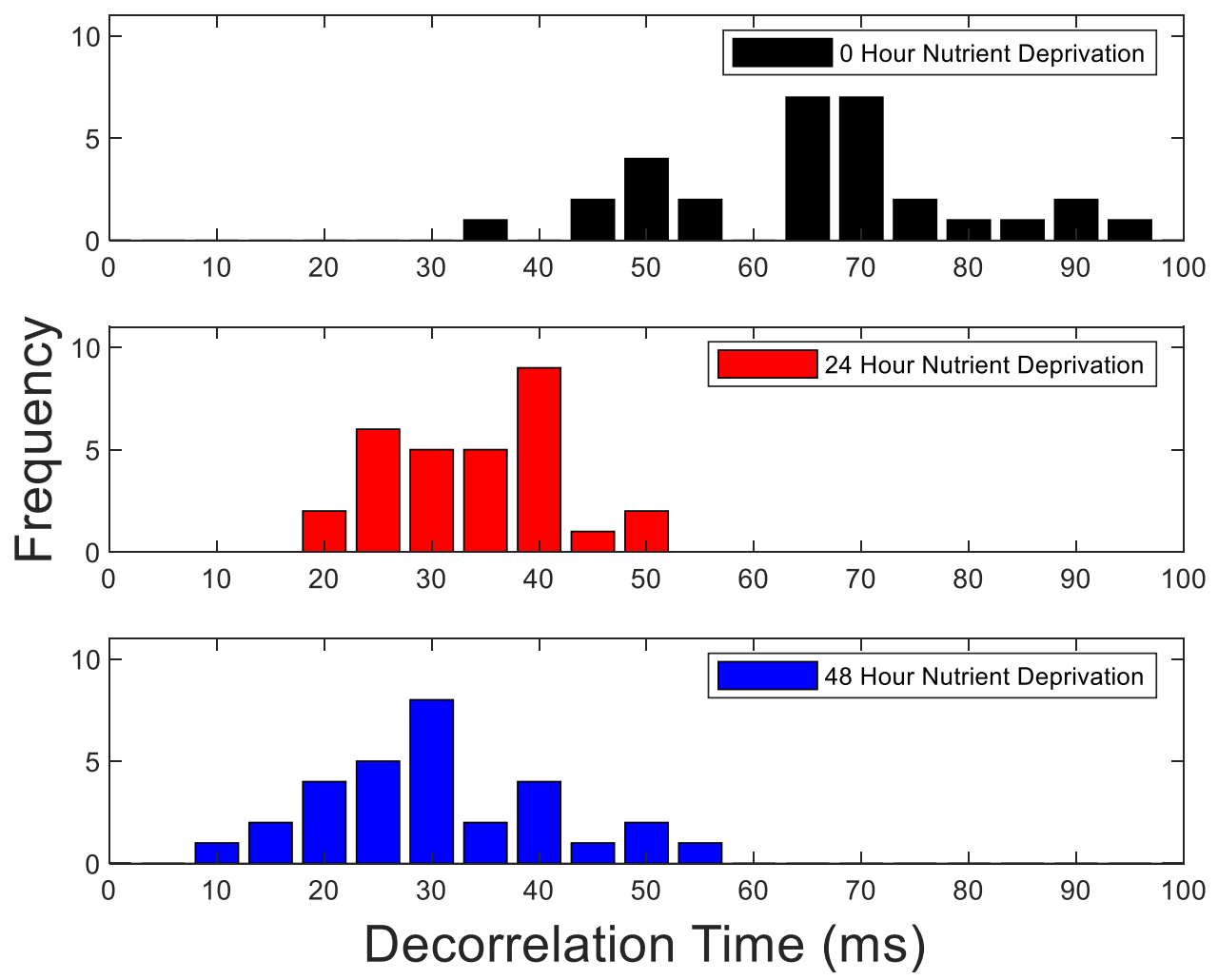

Figure 3-12. Decorrelation time frequency histograms for cells deprived of nutrients. For each treatment duration, 30 decorrelation times were acquired, with 10 from each experimental trial. Bins are 5 ms wide. 


\subsubsection{Cross-experiment Comparisons}

The results from the 24- and 48-hour paclitaxel-treated cells and the 24- and 48-hour nutrient-deprived cells were all compared to each other and it was observed that the differences in decorrelation time between the groups was not statistically significant in all cases $(\mathrm{p}>0.05)$. The DLS-OCT techniques used in this chapter could differentiate between untreated cells and treated cells but could not distinguish between the different types of treatment and also could not distinguish between treatment durations of 24 and 48 hours. 


\subsection{Using CONTIN to Determine Decorrelation Time Distributions}

\subsubsection{Paclitaxel-Treated Breast Cancer Cells}

Sample decorrelation time distributions from paclitaxel-treated cell pellets are displayed in Figure 3-13. The CONTIN algorithm produced distributions with three distinct peaks from all the ACFs, with no exceptions. Six metrics were used to compare the results between the untreated, 24 hour, and 48 hour treated groups: the area-under-the-curve (AUC) of each of the three peaks, and the mean decorrelation time $(\overline{D T})$ of each of the three peaks. The peaks are numbered 1-3 based on their position on the independent axis, from left to right.

The AUC of a peak is the intensity contribution from scatterers with decorrelation times within said peak expressed as a percentage of the overall fluctuating light intensity. Since the decorrelation time distributions are expressed in terms of the percentage contribution to intensity from each decorrelation time, the following condition holds true:

$$
A U C(\text { peak 1) }+A U C(\text { peak } 2)+A U C(\text { peak } 3)=100 \%
$$

The mean decorrelation time of each peak is a measure of the peak's position on the independent axis, and was calculated as follows:

$$
\overline{D T(\text { peak } i)}=\sum^{n} \frac{G(\tau)_{n} \tau_{n}}{A U C(\text { peak } i)} \text { for all } \tau_{n} \text { in peak } i
$$


The AUCs and $\overline{D T}$ s of each of the three peaks were compared between the 0 hour and 24 hour treated cells, and between the 0 hour and 48 hour treated cells from all ACFs from all experimental trials. Only two of the six metrics showed statistically significant changes $(\mathrm{p}<0.05)$ across all experimental trials: the AUC of the first peak, and the AUC of the third peak.

A significant increase was observed in the AUC of the first peak (see Table 3-3) from 0 hours to 24 and 48 hours, and a significant decrease was observed in the AUC of the third peak (see Table 3-4) from 0 hours to 24 and 48 hours. No statistically significant and repeatable changes were observed in any of the AUCs or $\overline{D T}$ s between the 24 and 48 hour treated groups. 

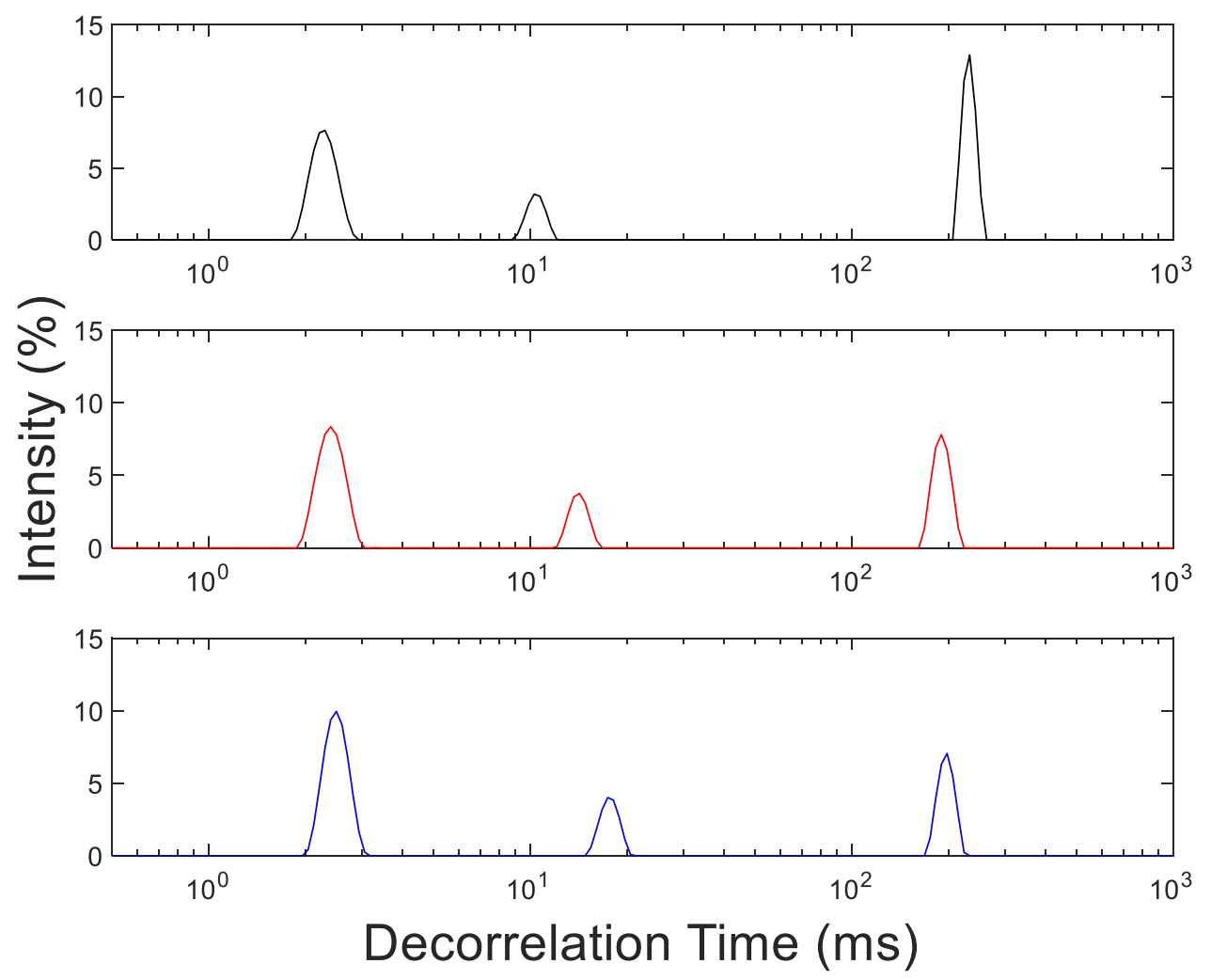

Figure 3-13. Sample decorrelation time distribution functions for cell pellets made from cells treated with 20 $\mathrm{ng} / \mathrm{mL}$ paclitaxel for various durations. Top: untreated cells, Centre: cells treated for 24 hours, Bottom: cells treated for 48 hours. Each of the above distributions came from one of the $30 \mathrm{ACF}$ acquired for each treatment duration. 
Table 3-3. Average AUCs the first peak of paclitaxel-treated cells. Each trial took place on a different week and used cells from a different passage number. In brackets are the percent changes in peak 1 AUC from 0 hours to either 24 or 48 hours.

\begin{tabular}{|l|l|l|l|}
\hline & Untreated & $\mathbf{2 4}$ hours & $\mathbf{4 8}$ hours \\
\hline Trial 1 & $48 \pm 3 \%$ & $\begin{array}{l}58 \pm 4 \% \\
(+20.0 \%)\end{array}$ & $\begin{array}{l}57 \pm 3 \% \\
(+18.2 \%)\end{array}$ \\
\hline Trial 2 & $46 \pm 3 \%$ & $\begin{array}{l}49 \pm 4 \% \\
(+7.0 \%)\end{array}$ & $\begin{array}{l}55 \pm 7 \% \\
(+20.9 \%)\end{array}$ \\
\hline Trial 3 & $41 \pm 5 \%$ & $\begin{array}{l}53 \pm 4 \% \\
(+30.0 \%)\end{array}$ & $\begin{array}{l}59 \pm 9 \% \\
(+45.2 \%)\end{array}$ \\
\hline
\end{tabular}

Table 3-4. Average AUCs the third peak of paclitaxel-treated cells. Each trial took place on a different week and used cells from a different passage number. In brackets are the percent changes in peak 3 AUC from 0 hours to either 24 or 48 hours.

\begin{tabular}{|c|c|l|l|}
\hline & Untreated & 24 hours & $\mathbf{4 8}$ hours \\
\hline Trial 1 & $38 \pm 3 \%$ & $29 \pm 3 \%$ & $24 \pm 4 \%$ \\
& & $(-22.8 \%)$ & $(-37.0 \%)$ \\
\hline Trial 2 & $40 \pm 4 \%$ & $33 \pm 3 \%$ & $27 \pm 7 \%$ \\
& & $(-16.8 \%)$ & $(-32.7 \%)$ \\
\hline Trial 3 & $42 \pm 4 \%$ & $29 \pm 3 \%$ & $25 \pm 5 \%$ \\
& & $(-31.6 \%)$ & $(-38.9 \%)$ \\
\hline
\end{tabular}




\subsubsection{Nutrient-Deprived Breast Cancer Cells}

Sample decorrelation time distributions from nutrient-deprived cell pellets are shown in Figure 3-14. As with the paclitaxel-treated cells, the CONTIN algorithm produced distributions with three distinct peaks from all the ACFs, with no exceptions. The AUC of each of the three peaks, and the $\overline{D T}$ of each of the three peaks were again compared between the 0 hour, 24 hour, and 48 hour nutrient-deprived cells.

The AUCs and $\overline{D T}$ s of each of the three peaks were compared between the 0 hour and 24 hour nutrient-deprived cells, and between the 0 hour and 48 hour nutrient-deprived cells from all ACFs from all experimental trials. Again, only two of the six metrics showed statistically significant changes $(\mathrm{p}<0.05)$ across all experimental trials: the AUC of the first peak, and the AUC of the third peak.

A significant increase was observed in the AUC of the first peak (see Table 3-5) from 0 hours to 24 and 48 hours, and a significant decrease was observed in the AUC of the third peak (see Table 3-6) from 0 hours to 24 and 48 hours. Again, no statistically significant and repeatable changes were observed in any of the AUCs or $\overline{D T}$ s between the 24 and 48 hour treated groups. 


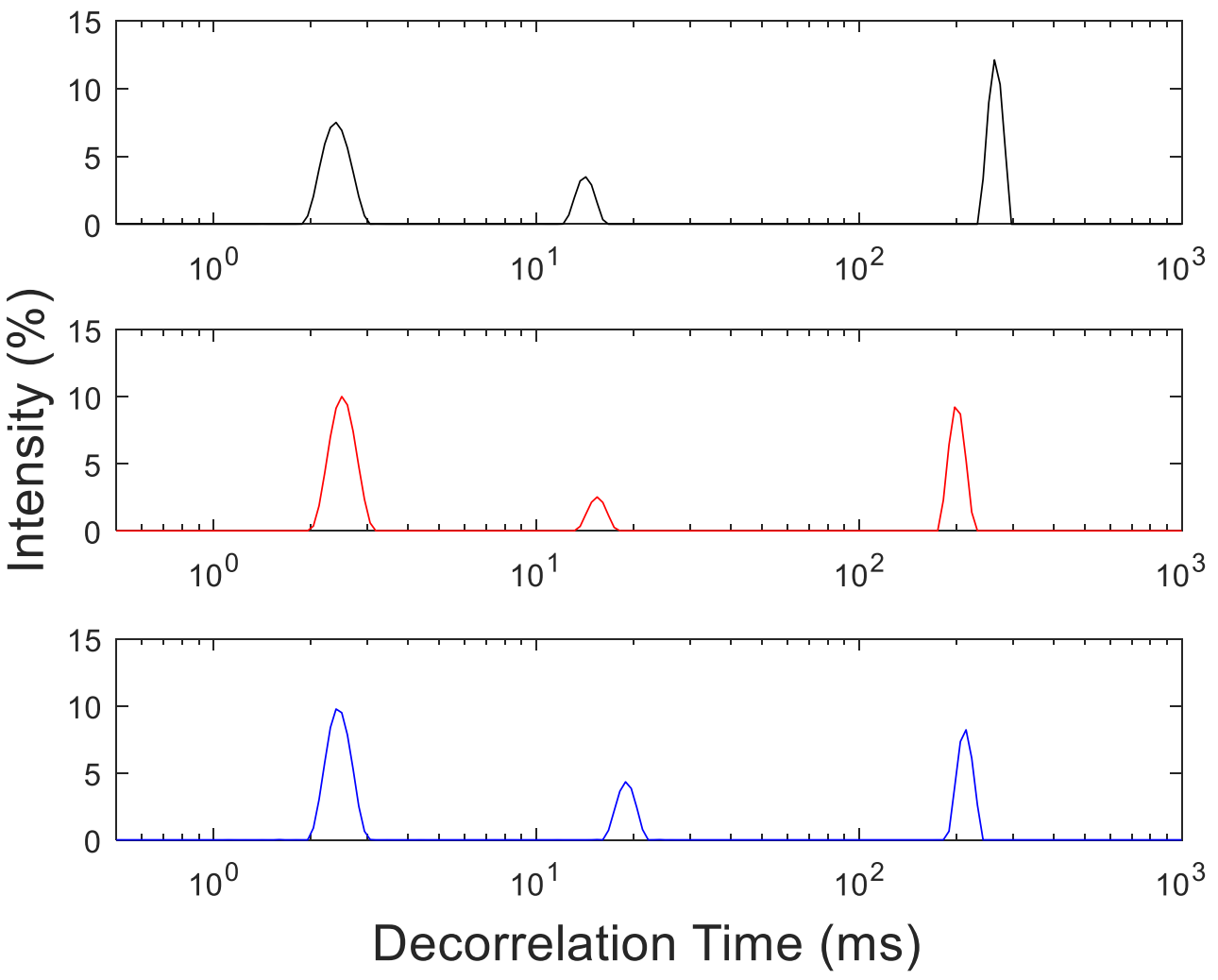

Figure 3-14. Sample decorrelation time distribution functions for cell pellets deprived of nutrients for various durations. Top: cells not deprived of nutrients, Centre: cells deprived of nutrients for 24 hours, Bottom: cells deprived of nutrients for 48 hours. Each of the above distributions came from one of the 30 ACFs acquired for each treatment duration. 
Table 3-5. Average AUCs the first peak of nutrient-deprived cells. Each trial took place on a different week and used cells from a different passage number. In brackets are the percent changes in peak 1 AUC from 0 hours to either 24 or 48 hours.

\begin{tabular}{|c|l|l|l|}
\hline & Untreated & $\mathbf{2 4}$ hours & $\mathbf{4 8}$ hours \\
\hline Trial 1 & $48 \pm 3 \%$ & $\begin{array}{l}57 \pm 4 \% \\
(+18.0 \%)\end{array}$ & $\begin{array}{l}56 \pm 3 \% \\
(+16.4 \%)\end{array}$ \\
\hline Trial 2 & $46 \pm 3 \%$ & $\begin{array}{l}62 \pm 3 \% \\
(+36.5 \%)\end{array}$ & $\begin{array}{l}59 \pm 3 \% \\
(+30.3 \%)\end{array}$ \\
\hline Trial 3 & $41 \pm 5 \%$ & $\begin{array}{l}48 \pm 3 \% \\
(+17.5 \%)\end{array}$ & $\begin{array}{l}60 \pm 10 \% \\
(+46.4 \%)\end{array}$ \\
\hline
\end{tabular}

Table 3-6. Average AUCs the third peak of nutrient-deprived cells. Each trial took place on a different week and used cells from a different passage number. In brackets are the percent changes in peak 3 AUC from 0 hours to either 24 or 48 hours.

\begin{tabular}{|c|c|l|l|}
\hline & Untreated & 24 hours & $\mathbf{4 8}$ hours \\
\hline Trial 1 & $38 \pm 3 \%$ & $\begin{array}{l}30 \pm 3 \% \\
(-22.7 \%)\end{array}$ & $\begin{array}{l}27 \pm 5 \% \\
(-30.4 \%)\end{array}$ \\
\hline Trial 2 & $40 \pm 4 \%$ & $\begin{array}{l}31 \pm 3 \% \\
(-23.4 \%)\end{array}$ & $\begin{array}{l}28 \pm 4 \% \\
(-30.7 \%)\end{array}$ \\
\hline Trial 3 & $42 \pm 4 \%$ & $30 \pm 4 \%$ & $28 \pm 6 \%$ \\
& & $(-27.7 \%)$ & $(-32.3 \%)$ \\
\hline
\end{tabular}




\subsubsection{Cross-experiment Comparisons}

The AUCs and $\overline{D T}$ s of each of the three peaks from the 24 - and 48-hour paclitaxeltreated cells and the 24- and 48-hour nutrient-deprived cells were all compared to each other. The differences in these metrics between the groups were either inconsistent or not statistically significant $(\mathrm{p}>0.05)$ in all trials. Once again, the techniques used in this chapter could be used to differentiate between untreated cells and treated cells but could not distinguish between the different types of treatment and could not distinguish between treatment durations of 24 and 48 hours.

\subsubsection{DLS-OCT Autocorrelation Function Curve Fitting}

In Chapter 3.2, the ACFs generated from OCT images of breast cancer cells were fitted to exponential decay functions. In Chapter 3.3, the CONTIN algorithm was used to generate decorrelation time distributions, which represent a series of multiple decorrelation times and their relative contributions to an overall ACF. This overall ACF can be expressed as a sum of multiple exponential decay functions. The sum of squared errors (SSEs) between the exponential fit curves and the experimental ACFs, and the SSEs between the CONTIN fit curves and the experimental ACFs, for all 150 acquired ACFs, are displayed in Table 3-7.

The curves generated using the CONTIN algorithm fitted the experimental data much better than the single exponential decay curves, with the prior having an SSE that was approximately 264 times lower than the latter, on average. CONTIN produced a better fit than the single exponential decay curve for all $150 \mathrm{ACFs}$, and the minimum ratio of the exponential decay SSE to the CONTIN SSE for a single ACF was approximately 74. An 
example of an ACF fitted to a single exponential decay fit is shown in Figure 3-15, and an example of that same ACF fitted to multiple exponential decay curves using CONTIN is shown in Figure 3-16.

Table 3-7. Mean sum-of-squared-error of the exponential fitted curves and the CONTIN fitted curves. SSEs were calculated from ACFs containing 250 data points from time lags between $0 \mathrm{~ms}$ to $747 \mathrm{~ms}$. In brackets are the standard deviations across 30 measurements.

\begin{tabular}{r|rr}
\multicolumn{1}{c}{ Treatment } & Mean SSE of Exponential Fit & Mean SSE of CONTIN Fit \\
\hline No treatment & $2.770(0.301)$ & $0.037(0.015)$ \\
24-hour Paclitaxel Exposure & $2.122(0.298)$ & $0.015(0.006)$ \\
48-hour Paclitaxel Exposure & $1.735(0.484)$ & $0.012(0.006)$ \\
24-hour Nutrient Deprivation & $2.131(0.435)$ & $0.017(0.009)$ \\
48-hour Nutrient Deprivation & $1.978(0.484)$ & $0.015(0.008)$
\end{tabular}




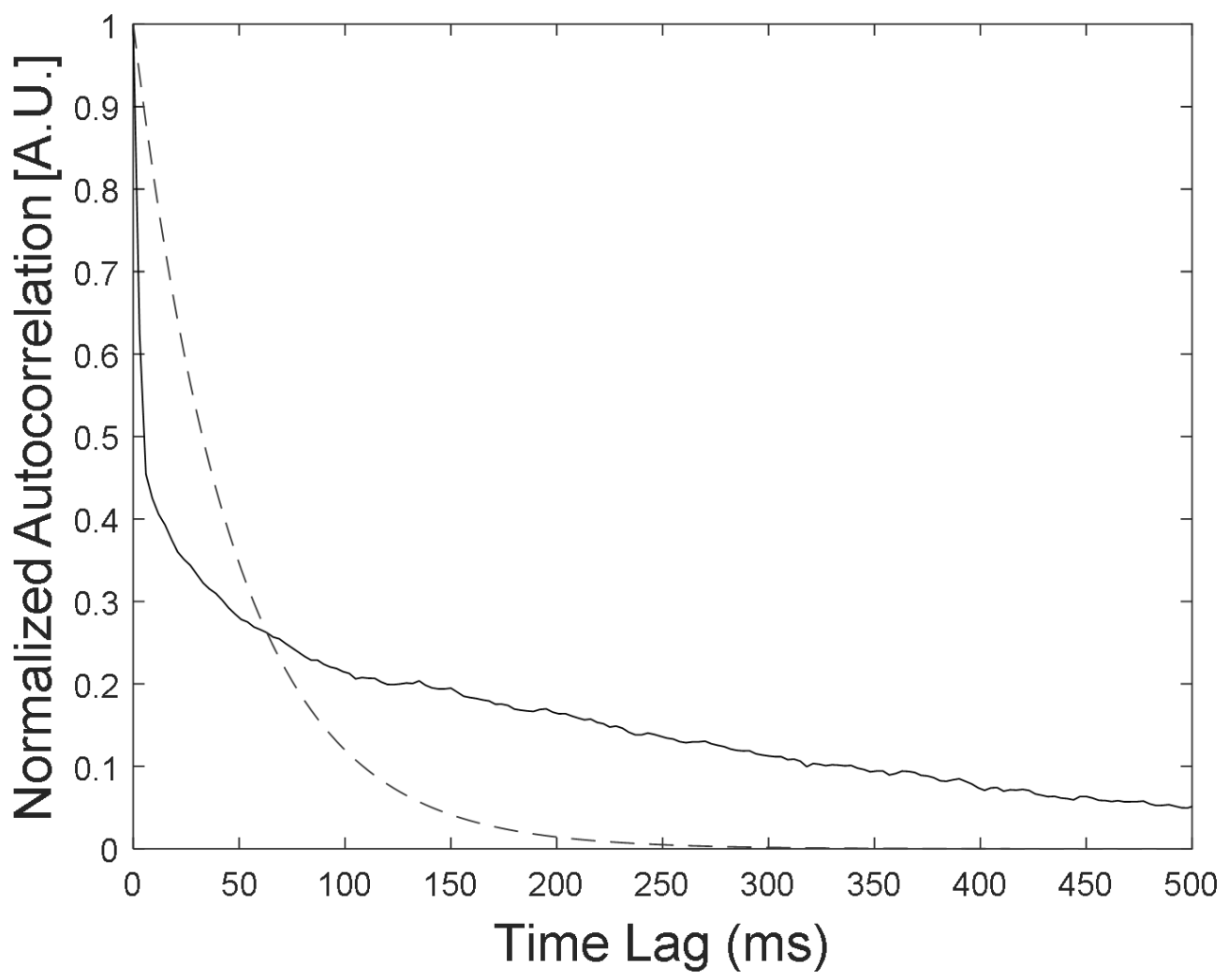

Figure 3-15. Exponential fit of a DLS-OCT ACF acquired from a pellet of untreated breast cancer cells. The experimental ACF is solid and the fitted curve is dashed. 


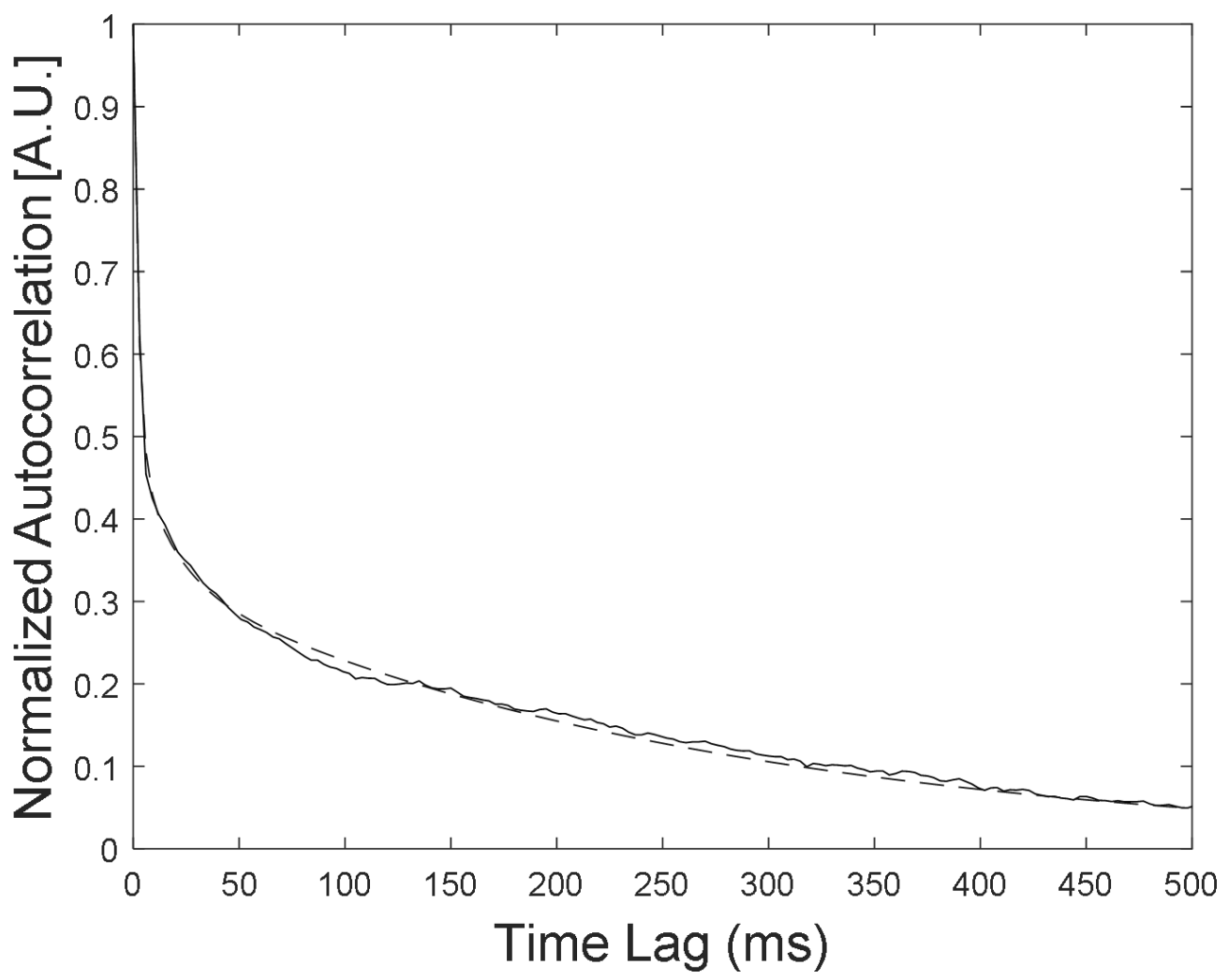

Figure 3-16. CONTIN fit of a DLS-OCT ACF acquired from untreated breast cancer cells. The experimental $A C F$ is solid and the fitted curve is dashed. 


\section{Chapter 4: Discussion}

The objectives of this thesis were to use DLS-OCT methods to detect cell death in adherent breast cancer cells and to use the CONTIN algorithm to produce a model that quantitatively describes the ACFs acquired from cell pellets. The hypothesis was that DLS-OCT could differentiate between untreated breast cancer cell populations and those treated with either chemotherapy or were deprived of media. The results of the experiments support the hypothesis and further validate the DLS-OCT methods first investigated by Farhat et al [43] by extending them to a new cell line and to different types of treatments and analysis methods.

In Chapter 3.2, it was shown that the DLS-OCT techniques previously used to detect cell death in AML cells could also differentiate between untreated and paclitaxel-treated breast cancer cells, and between untreated and media-deprived breast cancer cells. A significant drop in mean decorrelation time was observed in cell samples as early as 24 hours after the initiation of either paclitaxel treatment (Figure 3-8) or nutrient-deprivation (Figure 3-11). These results suggest an increase in intracellular motion in the chemotherapy-treated and nutrient-deprived groups, which we believe is indicative of the characteristic morphological changes that occur in apoptotic and oncotic cells.

Though the mean decorrelation time further decreased from 24 hours to 48 hours in all paclitaxel-treated and nutrient-deprived cell populations, this change was not statistically significant. Individual decorrelation times from the 24- and 48-hour treated cell populations varied, so even though the groups had different mean decorrelation times, there was still significant overlap in the decorrelation time frequency histograms of 24-hour and 48-hour 
treated cells (Figure 3-9 and Figure 3-12). We speculate that most of the cells treated with paclitaxel or deprived of media experienced the morphological changes associated with cell death within the first 24 hours of treatment, and that only a small number of cells underwent these changes between 24 and 48 hours after the start of treatment. Thus, the capability of the DLS-OCT methods investigated in this thesis to differentiate between dying and untreated cells could be subject to diminishing returns with increasing time between treatment and treatment monitoring.

The DLS-OCT methods were unable to differentiate between the nutrient-deprived and paclitaxel-treated populations as a similar decrease in decorrelation time was observed in both groups after 24 and 48 hours (see Table 3-1 and Table 3-2). In these experiments, the chemotherapy-treated cells were exposed to $20 \mathrm{ng} / \mathrm{mL}$ paclitaxel, and we predict that using a different concentration would affect the decorrelation times at 24 and 48 hours after the start of treatment. It has been shown that the concentration of paclitaxel administered to MCF-7 breast cancer cells is proportional to the fraction of cells that undergo apoptosis, albeit with diminishing returns [77], and we speculate that MCF-7 cells treated with lower concentrations of paclitaxel than $20 \mathrm{ng} / \mathrm{mL}$ would experience a smaller drop in decorrelation time on average.

In Chapter 3.3, the CONTIN algorithm was investigated as a potential tool for developing an equation to fit to DLS-OCT ACFs acquired from cancer cell pellets. The decorrelation time distribution functions computed by CONTIN for all cell pellets measured had exactly three peaks (examples in Figure 3-13 and Figure 3-14), and changes were observed between untreated and either nutrient-deprived or paclitaxel-treated cell samples: the AUC of the first peak was greater in treated cells than in untreated cells (Table 3-3 and 
Table 3-5), and the AUC of the third peak was smaller in treated cells than in untreated cells (Table 3-4 and Table 3-6). The fits to the experimental data using the CONTIN algorithm were just as sensitive as the standard exponential decay fit at differentiating between treated and untreated cell populations.

To our knowledge, we are the first to express the ACFs acquired from OCT imaging of in vitro cell pellets as superpositions of multiple exponential decay curves. Our model fits the experimental data significantly better than the single exponential decay model (see Figure 3-15 and Figure 3-16), suggesting that the ACFs arise from the superposition of light scattered by different structures within the cells. We speculate that the three peaks produced could correspond to decorrelation times from different organelles that have various sizes and movement speeds. For example, the third peak in the distribution, which corresponds to the largest decorrelation times, could result from light backscattered by the relatively stationary and large cellular nuclei, the second peak could result from mitochondria, and the first peak could be a result of smaller organelles such as lysosomes or even from scatterers moving in and out of the resolution volume.

This work is also significant because we have shown that DLS-OCT methods can differentiate between paclitaxel-treated and untreated cells, as well as between nutrientdeprived and untreated cells, in an adherent breast cancer cell line. In previous studies that used DLS-OCT to measure cell death it was speculated that decreases in decorrelation time after treatment resulted from increased intracellular motion due to apoptosis [43]. Apoptosis requires ATP, and cells that have been deprived of nutrients become depleted of ATP. By demonstrating that DLS-OCT methods can differentiate between untreated and nutrient- 
deprived cells, we have provided evidence suggesting that DLS-OCT can detect not only apoptosis, but also other modes of cell death.

To form pellets from adherent cells, the cells were treated with trypsin and EDTA to prevent cell adherence. We speculate that treating the cells with trypsin could have had an effect on the DLS-OCT measurements since trypsin cleaves proteins, and could have cleaved cytoskeletal fibers or other important intracellular proteins. A fractured cytoskeleton could affect intracellular motion, as mitochondria use cytoskeletal elements such as microtubules to move. We have demonstrated in this thesis that DLS-OCT could detect cell death in adherent cells despite all cell groups being treated with trypsin and EDTA prior to pellet formation.

There are some challenges unique to in vivo models that make it difficult to adapt DLS-OCT from in vitro to in vivo use for cell death monitoring. As discussed in Chapter 1.6, OCT cannot be used to non-invasively image tumors located deep within an organism due to the limited penetration depth of light; only tumors located on surface regions of an animal model or human patient would be suitable targets for DLS-OCT monitoring. The flow of blood, which is present in in vivo models but not in the in vitro cell populations, can have a large effect on DLS measurements as blood cells are rapidly moving light scatterers. Mariampillai et al were able to generate images of vasculature using OCT, and it may be possible use image processing techniques to separate blood vessels from cellular regions. Farhat et al investigated the use of DLS-OCT to detect cancer cell death in mice implanted with bladder cancer tumors and found that the average decorrelation time increased after 24 and 48 hour chemotherapy exposure, the opposite of what was observed in the in vitro experiments in which the decorrelation time dropped after chemotherapy exposure, though 
concluded that the results were inconclusive as only three mice were used in the experiments [78].

Future experiments involving the use of DLS-OCT to detect cell death should aim to validate the results of DLS-OCT measurements by quantifying the percentage of dead or dying cells in a population after a treatment is administered. Furthermore, attempts should be made to classify the mechanisms of cell death within cell populations to further investigate whether DLS-OCT can differentiate between apoptotic and oncotic cells. Some examples of methods for identifying cell death are histological staining, which can be used to estimate the percentage of dead cells in a population, and flow cytometry, which can detect specific forms of cell death such as apoptosis, in large quantities of cells [79].

Techniques such as confocal microscopy can be used to track the movement speed of organelles such as mitochondria or nuclei using various staining agents [80]. Studying the movement of organelles in untreated and treated cells can lead to a better understanding of the biological processes that contribute to the decrease in decorrelation time observed in treated cells. If the movement speeds of intracellular scatterers is quantified, the decorrelation times can be estimated using Equation 11, providing insights about the three peaks found in each CONTIN decorrelation time distribution.

Future studies should also aim to compare the results of DLS-OCT analysis of cell samples across different cell lines and OCT devices. The decorrelation time distributions found in this study consisted of three distinct peaks, but it is unknown if this is universal or if a different cell line, or a different OCT device would produce slightly different ACFs with different decorrelation times. 


\section{Appendix}

\section{Light Microscopy Images of In Vitro Cells}

During the third repetition of the in vitro experiments, approximately $3 * 10^{6}$ breast cancer cells not used to make pellets were equally divided into three $25 \mathrm{~cm}^{2}$ flasks and incubated for 24 hours. After this period, the flasks were imaged with a standard light microscope. The media in one of the flasks was replaced with 1x PBS to emulate the effects of nutrient-deprivation, and another flask was treated with $20 \mathrm{ng} / \mathrm{mL}$ paclitaxel. All three flasks were imaged with a light microscope again after 24 hours and 48 hours (see Figure A$1)$.

Though the light microscopy images do not explicitly confirm the presence of apoptotic or oncotic cells, they do provide evidence that the cells responded to the treatments. In the images of untreated cells, a monolayer of tightly-packed cells is clearly visible, and only a few cells are suspended in the media. In the images of cells treated with paclitaxel, a monolayer is still visible, but it has reduced confluence, and there are considerably more suspended cells and irregularly-shaped cells. In the images of nutrient-deprived cells, there is no longer a visible adherent monolayer and all the cells are suspended in the PBS. Suspended cells could be symptomatic of apoptosis or oncosis, as dead cells lose their adherence. 

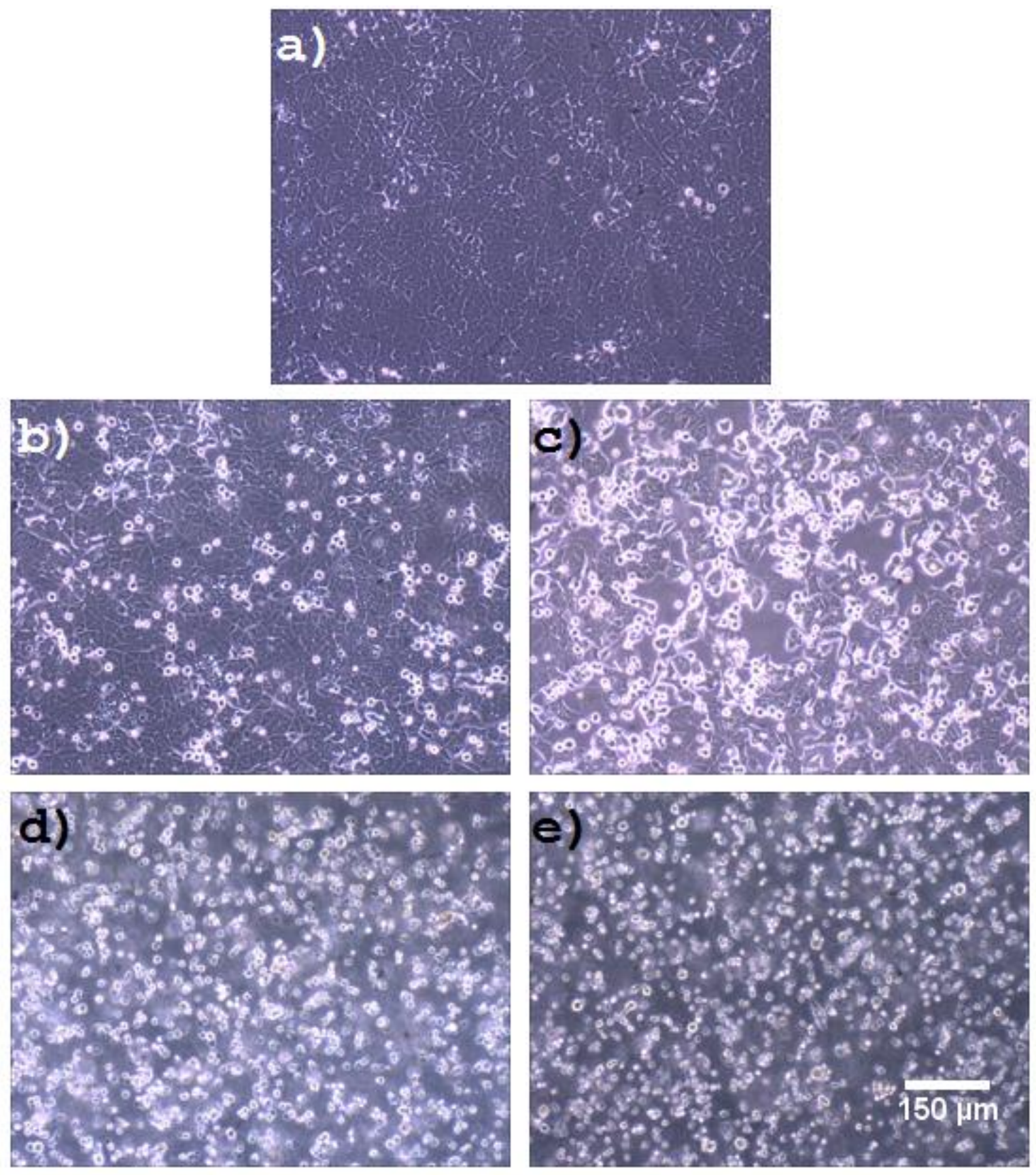

Figure A-1. Light microscopy images of MCF-7 breast cancer cells. a) untreated cells, b) cells exposed to 20 $\mathrm{ng} / \mathrm{mL}$ paclitaxel for 24 hours, $c$ ) cells exposed to $20 \mathrm{ng} / \mathrm{mL}$ paclitaxel for 24 hours, d) cells deprived of media and placed in PBS for 24 hours, and e) cells deprived of media and placed in PBS for 48 hours. 


\section{References}

[1] H. Varmus and R. A. Weinberg, Genes and the biology of cancer. New York: Scientific American Library, 1993.

[2] D. Hanahan and R. A. Weinberg, "The hallmarks of cancer.," Cell, vol. 100, no. 1, pp. 57-70, 2000.

[3] Y. Kato et al., "Acidic extracellular microenvironment and cancer," Cancer Cell Int., vol. 13, no. 1, p. 1, 2013.

[4] Canadian Cancer Society's Advisory Committee on Cancer Statistics, "Canadian Cancer Statistics 2017," Can. Cancer Soc., pp. 1-132, 2017.

[5] U. E. N. C. for E. Assessment, "SEER cancer statistics review, 1975-2005," Natl. Cancer Institute. Bethesda, MD, pp. 1992-2013, 2009.

[6] Statistics Canada, "Populations Projections: Canada, the provinces and territories, 2013 to 2063," Dly., pp. 1-4, 2014.

[7] R. Sullivan et al., "Global cancer surgery: Delivering safe, affordable, and timely cancer surgery," Lancet Oncol., vol. 16, no. 11, pp. 1193-1224, 2015.

[8] J. F. R. Kerr, a H. Wyllie, and a R. Currie, "Apoptasis: A Basic Biological Phenomenon with Wideranging Applications in Tissue Kinetics," Wide-Ranging Implic. Tissue Kinet. Br. J. Cancer, vol. 26, no. 4, pp. 23-257, Aug. 1972.

[9] M. Lamkanfi and V. M. Dixit, "Manipulation of host cell death pathways during microbial infections.," Cell Host Microbe, vol. 8, no. 1, pp. 44-54, Jul. 2010.

[10] E. Gulbins, S. Dreschers, and J. Bock, "Role of mitochondria in apoptosis," Exp. Physiol., vol. 88, no. 1, pp. 85-90, 2003.

[11] S. L. Fink and B. T. Cookson, "Apoptosis, Pyroptosis, and Necrosis: Mechanistic Description of Dead and Dying Eukaryotic Cells," Infect. Immun., vol. 73, no. 4, pp. 1907-1916, 2005.

[12] G. Majno and I. Joris, "Apoptosis, Oncosis, and Necrosis. An Overview of Cell Death,” Am. J. ofPathology, vol. 146, no. 1, 1995.

[13] H. Vakifahmetoglu, M. Olsson, and B. Zhivotovsky, "Death through a tragedy: Mitotic catastrophe," Cell Death Differ., vol. 15, no. 7, pp. 1153-1162, 2008.

[14] F. Ianzini and M. A. Mackey, "Spontaneous premature chromosome condensation and mitotic catastrophe following irradiation of HeLa S3 cells," Int. J. Radiat. Biol., vol. 72, no. 4, pp. 409-421, 1997.

[15] National Institute of General Medical Sciences, "Inside the Cell," 2005.

[16] J. Widder, "The origins of radiotherapy: Discovery of biological effects of X-rays by Freund in 1897, Kienböck's crucial experiments in 1900 and still it is the dose," Radiother. Oncol., 
vol. 112, no. 1, pp. 150-152, 2014.

[17] J. Bernier, E. J. Hall, and A. Giaccia, "Radiation oncology: A century of achievements," Nat. Rev. Cancer, vol. 4, no. 9, pp. 737-747, 2004.

[18] A. H. W. Nias, "Radiobiology for the radiologist (3rd edition)," Int. J. Radiat. Biol., vol. 54, no. 3, pp. 505-506, 1988.

[19] A. B. Weisse, Medical odysseys: the different and sometimes unexpected pathways to twentieth-century medical discoveries. New Brunswick: Rutgers University Press, 1992.

[20] V. T. Devita and E. Chu, "A History of Cancer Chemotherapy," Cancer Res, vol. 68, no. 21, pp. 8643-53, 2008.

[21] R. Jones, "Cytotoxic chemotherapy: clinical aspects," 2016.

[22] J. J. Field, A. Kanakkanthara, and J. H. Miller, "Microtubule-targeting agents are clinically successful due to both mitotic and interphase impairment of microtubule function," Bioorg. Med. Chem., vol. 22, pp. 5050-5059, 2014.

[23] G. H. Williams and K. Stoeber, "The cell cycle and cancer," J. Pathol., vol. 226, no. 2, pp. 352-364, 2012.

[24] P. Therasse et al., "New Guidelines to Evaluate the Response to Treatment in Solid Tumors," J. Natl. Cancer Inst., vol. 92, no. 3, pp. 205-216, 2000.

[25] A. Sohaib, “RECIST rules,” Cancer Imaging, vol. 12, no. 2, pp. 345-346, 2012.

[26] M. Vander Heiden, L. Cantley, and C. Thompson, "Understanding the Warburg effect: The metabolic Requiremetns of cell proliferation," Science (80-. )., vol. 324, no. 5930, pp. 10291033, 2009.

[27] G. Muehllehner and J. S. Karp, "Positron emission tomography," Phys. Med. Biol., vol. 51, no. 13, pp. 117-137, 2006.

[28] B. Woesler et al., "Non-invasive grading of primary brain tumours: results of a comparative study between SPET with 123I-alpha-methyl tyrosine and PET with 18F-deoxyglucose.," Eur. J. Nucl. Med., vol. 24, no. 4, pp. 428-34, Apr. 1997.

[29] C. Plathow and W. A. Weber, "Tumor cell metabolism imaging.," J. Nucl. Med., vol. 49 Suppl 2, no. Suppl 2, p. 43S-63S, Jun. 2008.

[30] R. Gruetter et al., "Direct measurement of brain glucose concentrations in humans by 13C NMR spectroscopy.," Proc. Natl. Acad. Sci. U. S. A., vol. 89, no. 3, pp. 1109-12, Feb. 1992.

[31] J. L. Tatum, "Hypoxia: Importance in tumor biology, noninvasive measurement by imaging, and value of its measurement in the management of cancer therapy," Int. J. Radiat. Biol., vol. 82, no. 10, pp. 699-757, 2006.

[32] L. Frullano and T. J. Meade, "Multimodal MRI contrast agents," J. Biol. Inorg. Chem., vol. 12, no. 7, pp. 939-949, 2007. 
[33] P. Dawson, "Functional imaging in CT," Eur. J. Radiol., vol. 60, no. 3, pp. 331-340, 2006.

[34] U. Emilio Quaia, "Microbubble ultrasound contrast agents: an update," Eur Radiol, vol. 17, pp. 1995-2008, 2007.

[35] A. Kumar et al., "Doppler ultrasound scoring to predict chemotherapeutic response in advanced breast cancer," World J. Surg. Oncol., vol. 5, no. 5, 2007.

[36] U. E. Martinez-Outschoorn, Z. Lin, D. Whitaker-Menezes, A. Howell, F. Sotgia, and M. P. Lisanti, "Ketone body utilization drives tumor growth and metastasis," Cell Cycle, vol. 11, no. 21, pp. 3964-3971, Nov. 2012.

[37] T. Belhocine et al., "Increased Uptake of the Apoptosis-imaging Agent 99m Tc Recombinant Human Annexin V in Human Tumors after One Course of Chemotherapy as a Predictor of Tumor Response and Patient Prognosis 1," Clin. Cancer Res., vol. 8, no. September, pp. 2766$2774,2002$.

[38] G. J. Czarnota et al., "Ultrasound imaging of apoptosis: High-resolution non-invasive monitoring of programmed cell death in vitro, in situ and in vivo," Br. J. Cancer, vol. 81, no. 3, pp. 520-527, Oct. 1999.

[39] R. M. Vlad, R. K. Saha, N. M. Alajez, S. Ranieri, G. J. Czarnota, and M. C. Kolios, “An increase in cellular size variance contributes to the increase in ultrasound backscatter during cell death," Ultrasound Med. Biol., vol. 36, no. 9, pp. 1546-1558, 2010.

[40] A. Sadeghi-Naini et al., "Chemotherapy-Response Monitoring of Breast Cancer Patients Using Quantitative Ultrasound-Based Intra-Tumour Heterogeneities," Sci. Rep., vol. 7, no. 1, 2017.

[41] UBC Biomedical Engineering, "Research - Optical Cancer Imaging LabOptical Cancer Imaging Lab." [Online]. Available: http://biophotonics.bccrc.ca/index.php/research/. [Accessed: 18-May-2018].

[42] G. Farhat, V. X. D. Yang, G. J. Czarnota, and M. C. Kolios, "Detecting cell death with optical coherence tomography and envelope statistics," J. Biomed. Opt., 2011.

[43] G. Farhat, A. Mariampillai, V. X. D. Yang, G. J. Czarnota, and M. C. Kolios, "Detecting apoptosis using dynamic light scattering with optical coherence tomography," J. Biomed. Opt., vol. 16, no. 7, p. 070505, 2011.

[44] M. E. Brezinski, Optical coherence tomography: Principles and applications. Academic Press, 2006.

[45] A. F. Fercher, W. Drexler, C. K. Hitzenberger, and T. Lasser, "Optical coherence tomography_principles and applications," Rep. Prog. Phys, vol. 66, no. 03, pp. 239-303, 2003.

[46] W. I. Goldburg, "Dynamic light scattering,” Am. J. Phys., vol. 67, no. 12, p. 1152, Nov. 1999.

[47] B. J. Berne and R. Pecora, Dynamic Light Scattering: With Applications to Chemistry, Biology, and Physics. Dover Publications, 1976. 
[48] N. A. Clark, J. H. Lunacek, and G. B. Benedek, "A Study of Brownian Motion Using Light Scattering," Am. J. Phys., vol. 38, no. 5, pp. 575-585, May 1970.

[49] D. E. Koppel, "Analysis of macromolecular polydispersity in intensity correlation spectroscopy: The method of cumulants," J. Chem. Phys., vol. 57, no. 11, pp. 4814-4820, 1972.

[50] B. J. Frisken, "Revisiting the method of cumulants for the analysis of dynamic light-scattering data," Appl. Opt., vol. 40, no. 24, p. 4087, 2001.

[51] S. W. Provencher, "A constrained regularization method for inverting data represented by linear algebraic or integral equations," Comput. Phys. Commun., vol. 27, no. 3, pp. 213-227, 1982.

[52] S. W. Provencher, "Contin - a General-Purpose Constrained Regularization Program for Inverting Noisy Linear Algebraic and Integral-Equations," Comput. Phys. Commun., vol. 27, no. 3, pp. 229-242, 1982.

[53] A. Scotti et al., "The CONTIN algorithm and its application to determine the size distribution of microgel suspensions," J. Chem. Phys., vol. 142, no. 23, p. 234905, 2015.

[54] "Using DLS Deconvolution Algorithms to Acquire Intensity Weighted Particle Size Distribution from Measured Correlogram," 2014. [Online]. Available: https://www.newsmedical.net/whitepaper/20141221/Using-DLS-Deconvolution-Algorithms-to-AcquireIntensity-Weighted-Particle-Size-Distribution-from-Measured-Correlogram.aspx. [Accessed: 06-Jun-2018].

[55] I.-G. Marino, "rilt - File Exchange - MATLAB Central," 2004. [Online]. Available: https://www.mathworks.com/matlabcentral/fileexchange/6523-rilt. [Accessed: 06-Jun-2018].

[56] J. Lee, W. Wu, J. Y. Jiang, B. Zhu, and D. A. Boas, "Dynamic light scattering optical coherence tomography," Opt. Express, vol. 20, no. 20, p. 22262, Sep. 2012.

[57] J. Lee et al., "Quantitative Imaging of Cerebral Blood Flow Velocity and Intracellular Motility using Dynamic Light Scattering-Optical Coherence Tomography," J. Cereb. Blood Flow Metab., vol. 33, no. 6, pp. 819-825, Jun. 2013.

[58] R. L. Blackmon et al., "Imaging Extracellular Matrix Remodeling in Vitro by DiffusionSensitive Optical Coherence Tomography," Biophys. J., vol. 110, no. 8, pp. 1858-1868, 2016.

[59] N. Weiss, K. el Tayeb el Obied, J. Kalkman, R. G. H. Lammertink, and T. G. van Leeuwen, "Measurement of biofilm growth and local hydrodynamics using optical coherence tomography," Biomed. Opt. Express, vol. 7, no. 9, pp. 3508-3518, Sep. 2016.

[60] H. C. Van de Hulst, Light scattering by small particles. Dover Publications, 1981.

[61] G. W. Petty, A First Course in Atmospheric Radiation (2nd Ed.). Sundog Pub, 2006.

[62] "Blue Sky and Rayleigh Scattering." [Online]. Available: http://people.whitman.edu/ dobson/RayScat.html. [Accessed: 24-May-2018].

[63] N. A. Campbell, Biology: Concepts and Connections. 2008. 
[64] I. E. Scheffler, Mitochondria. Wiley-Liss, 2008.

[65] J. G. H. Joosten, J. L. McCarthy, and P. N. Pusey, "Dynamic and static light scattering by aqueous polyacrylamide gels," Macromolecules, vol. 24, no. 25, pp. 6690-6699, 1991.

[66] "How Is Acute Myeloid Leukemia Diagnosed?," American Cancer Society. [Online]. Available: https://www.cancer.org/cancer/acute-myeloid-leukemia/detection-diagnosisstaging/how-diagnosed.html. [Accessed: 25-May-2018].

[67] W. Kühnel and W. Kühnel, Color atlas of cytology, histology, and microscopic anatomy. Thieme, 2003.

[68] P. Y. Liu et al., "Cell refractive index for cell biology and disease diagnosis: past, present and future," Lab Chip, 2016.

[69] W. Choi et al., "Tomographic phase microscopy," Nat. Methods, vol. 4, no. 9, pp. 717-719, 2007.

[70] K. Haseda, K. Kanematsu, K. Noguchi, H. Saito, N. Umeda, and Y. Ohta, "Significant correlation between refractive index and activity of mitochondria: single mitochondrion study," vol. 6, no. 3, pp. 717-719, 2015.

[71] J. D. Wilson, W. J. Cottrell, and T. H. Foster, "Index-of-refraction-dependent subcellular light scattering observed with organelle-specific dyes," J. Biomed. Opt., vol. 12, no. 1, p. 014010 , 2007.

[72] R. M. Pasternack, J.-Y. Zheng, and N. N. Boustany, "Optical scatter changes at the onset of apoptosis are spatially associated with mitochondria.," J. Biomed. Opt., vol. 15, no. 4, p. 040504, 2014.

[73] M. W. Laschke, B. Vollmar, and M. D. Menger, "The dorsal skinfold chamber: Window into the dynamic interaction of biomaterials with their surrounding host tissue," Eur. Cells Mater., vol. 22, pp. 147-167, 2011.

[74] A. Zam and M. C. Kolios, "Measuring intracellular motion in cancer cell using optical coherence tomography," vol. 9707, p. 97070V, 2016.

[75] A. Miglietta, M. L. Panno, F. Bozzo, L. Gabriel, and C. Bocca, "Insulin can modulate MCF7 cell response to paclitaxel," Cancer Lett., vol. 209, no. 2, pp. 139-145, 2004.

[76] J. H. Kim et al., "Paclitaxel suppresses the viability of breast tumor MCF7 cells through the regulation of EF1á and FOXO3a by AMPK signaling," Int. J. Oncol., vol. 47, no. 5, pp. 1874$1880,2015$.

[77] D. E. Saunders, W. D. Lawrence, C. Christensen, N. L. Wappler, H. Ruan, and G. Deppe, "Paclitaxel-Induced Apoptosis in MCF-7 Breast-Cancer Cells," Int. J. Cancer, vol. 70, pp. 214-220, 1997.

[78] G. Farhat, A. Mariampillai, K. K. K. C. C. Lee, V. X. D. V. X. D. Yang, G. J. G. J. Czarnota, and M. C. Kolios, "Measuring intracellular motion using dynamic light scattering with optical coherence tomography in a mouse tumor model," SPIE BiOS, vol. 8230, pp. 823002-823007, 2012. 
[79] D. Wlodkowic, J. Skommer, and Z. Darzynkiewicz, "Flow cytometry-based apoptosis detection.," Methods Mol. Biol., vol. 559, pp. 19-32, 2009.

[80] N. Beraud et al., "Mitochondrial dynamics in heart cells: Very low amplitude high frequency fluctuations in adult cardiomyocytes and flow motion in non beating Hl-1 cells," J. Bioenerg. Biomembr., vol. 41, no. 2, pp. 195-214, 2009. 